\title{
Synthesis and Protection of Aryl Sulfates using the 2,2,2-Trichloroethyl Moiety
}

Yong Liu, I-Feh Felicia Lien, Scott Ruttgaizer, Peter Dove and Scott D. Taylor*

Department of Chemistry, University of Waterloo, 200 University Avenue

West, Waterloo, Ontario, Canada, N2L $3 G 1$.

\section{Supporting Information}

General. All starting materials and reagents were obtained from Aldrich Chemical Company (Oakville, ON, Canada). Solvents were purchased from Caledon Laboratories (Georgetown, Ontario, Canada) or BDH Canada (Toronto, Canada). THF was distilled over $\mathrm{Na}$ /benzophenone under argon. $\mathrm{CH}_{2} \mathrm{Cl}_{2}$ was distilled from calcium hydride under argon. All columns were run using silica gel (40-60 A, 230-400 mesh) obtained from Silicycle, (Laval, Quebec, Canada). ${ }^{1} \mathrm{H},{ }^{19} \mathrm{~F}$, and ${ }^{13} \mathrm{C}$ NMR spectra were recorded on a Bruker Avance 300 spectrometer. ${ }^{19} \mathrm{~F}$ and ${ }^{13} \mathrm{C}$ NMR were ${ }^{1} \mathrm{H}$ decoupled unless stated otherwise. Chemicals shifts ( $\delta$ ) for ${ }^{1} \mathrm{H}$ NMR spectra run in $\mathrm{CDCl}_{3}$ are reported in ppm relative to the internal standard tetramethylsilane (TMS). Chemical shifts $(\delta)$ for ${ }^{1} \mathrm{H}$ NMR spectra run in $\mathrm{D}_{2} \mathrm{O}$ are reported in ppm relative to residual solvent protons $(\delta 4.79)$. For ${ }^{13} \mathrm{C} \mathrm{NMR} \mathrm{spectra} \mathrm{run} \mathrm{in} \mathrm{CDCl}_{3}$, chemical shifts are reported in ppm relative to the $\mathrm{CDCl}_{3}$ residual carbons ( $\delta 77.0$ for central peak). For ${ }^{13} \mathrm{C}$ NMR spectra run in $\mathrm{D}_{2} \mathrm{O}$, chemical shifts are reported in ppm relative to $\mathrm{CH}_{3} \mathrm{OH}$ in $\mathrm{D}_{2} \mathrm{O}$ (external). For ${ }^{19} \mathrm{~F}$ NMR spectra, chemical shifts are reported in ppm relative to $\mathrm{CFCl}_{3}$ (external). Low-resolution (LRMS) and high-resolution (HRMS) electron impact (EI) mass spectra were obtained on a Micromass 70-S-250 sector mass spectrometer. Low-resolution and high-resolution electrospray ionization (ESI) mass spectra were obtained on an ABI/Sciex Qstar mass spectrometer. All ESIMS were run in the negative ion mode unless stated otherwise. Melting points were determined on Fisher-Johns melting point apparatus and are uncorrected.

General procedure for the synthesis of trichloroethyl esters 16-26 and 39. To a solution of the phenol (5-14 or 38, $500 \mathrm{mg}), 1.2$ equiv $\mathrm{Et}_{3} \mathrm{~N}$ and 1.0 equiv DMAP in dry THF $(20 \mathrm{~mL})$ was added dropwise over a period of 15 minutes a solution of chlorosulfuric acid 2,2,2-trichloroethyl ester (4) in dry THF (10 mL). For estrone (15), 2 equiv reagent 4, 2 equiv $\mathrm{Et}_{3} \mathrm{~N}$ and 1 equiv DMAP were used. The solution was stirred for $10 \mathrm{~h}$. EtOAc was added $(100 \mathrm{~mL})$ and the solution washed with $\mathrm{H}_{2} \mathrm{O}(40 \mathrm{~mL}), 0.5 \mathrm{~N} \mathrm{HCl}(2 \times 40 \mathrm{~mL}), \mathrm{H}_{2} \mathrm{O}(40 \mathrm{~mL})$ and sat. brine $(40$ $\mathrm{mL})$. The organic layer was dried $\left(\mathrm{Na}_{2} \mathrm{SO}_{4}\right)$, and concentrated by rotary evaporation. Pure product was obtained by flash chromatography of the residue using 5\% EtOAc/Hexane or 10\% EtOAc/Hexane. Yields are listed in Table 1 of the main manuscript.

Sulfuric acid, 4-fluorophenyl ester 2,2,2-trichloroethyl ester (16). White semi-solid at rt; ${ }^{1} \mathrm{H}$ NMR $\left(\mathrm{CDCl}_{3}\right) \delta$ 7.30-7.38 (m, 2H), 7.05-7.16 (m, 2H), $4.83(\mathrm{~s}, 2 \mathrm{H}) ;{ }^{19} \mathrm{~F} \mathrm{NMR}\left(\mathrm{CDCl}_{3}\right) \delta-112.7$;

${ }^{13} \mathrm{C} \mathrm{NMR}\left(\mathrm{CDCl}_{3}\right) \delta 161.4(\mathrm{~d}, J=248 \mathrm{~Hz}), 145.8(\mathrm{~d}, J=3.0 \mathrm{~Hz}), 123.1(\mathrm{~d}, J=9 \mathrm{~Hz}), 116.9(\mathrm{~d}, J$ 
$=24 \mathrm{~Hz}$ ), 92.4, 80.5; (LRMS (EI), m/z (relative intensity), 324 (7), 205 (12), 192 (26), 132 (14), 112 (100); HRMS (EI) calcd. for $\mathrm{C}_{8} \mathrm{H}_{6} \mathrm{Cl}_{3} \mathrm{FO}_{4} \mathrm{~S} 321.9036$, found 321.9030 .

Sulfuric acid, phenyl ester 2,2,2-trichloroethyl ester (17). White solid; mp $25-27^{\circ} \mathrm{C} ;{ }^{1} \mathrm{H}$ NMR $\left(\mathrm{CDCl}_{3}\right) \delta$ 7.30-7.50 (m, 5H), $4.82(\mathrm{~s}, 2 \mathrm{H}) ;{ }^{13} \mathrm{C} \mathrm{NMR}\left(\mathrm{CDCl}_{3}\right) \delta 150.0,130.1,127.9,121.0,92.3$, 80.3; LRMS (EI), m/z (relative intensity), 306 (10), 232 (7), 187 (9), 173 (22), 130 (14), 107 (16), 94 (100); HRMS (EI) calcd. for $\mathrm{C}_{8} \mathrm{H}_{7} \mathrm{Cl}_{3} \mathrm{O}_{4} \mathrm{~S}$ 303.9131, found 303.9137.

Sulfuric acid, 4-nitrophenyl ester 2,2,2-trichloroethyl ester (18). Slightly yellow solid; $\mathrm{mp}$ $82-84^{\circ} \mathrm{C}\left(\right.$ lit $\left.^{1}: 85^{\circ} \mathrm{C}\right) ;{ }^{1} \mathrm{H}$ NMR $\left(\mathrm{CDCl}_{3}\right) \delta 8.36(\mathrm{~d}, J=9.1 \mathrm{~Hz}, 2 \mathrm{H}), 7.55(\mathrm{~d}, J=9.1 \mathrm{~Hz}, 2 \mathrm{H}), 4.89$ $(\mathrm{s}, 2 \mathrm{H}) ;{ }^{13} \mathrm{C} \mathrm{NMR}\left(\mathrm{CDCl}_{3}\right) \delta 153.8,146.5,125.8,121.9,91.9,80.7$; LRMS (EI), $\mathrm{m} / z$ (relative intensity), 349 (7), 232 (22), 219 (20), 139 (100), 131 (50); HRMS (EI) calcd. for $\mathrm{C}_{8} \mathrm{H}_{6} \mathrm{Cl}_{3} \mathrm{NO}_{6} \mathrm{~S}$ 348.8981 , found 348.8974 .

Sulfuric acid, [4-trifluoromethyl]phenyl ester 2,2,2-trichloroethyl ester (19). White solid; $\mathrm{mp}$ $42-43{ }^{\circ} \mathrm{C} ;{ }^{1} \mathrm{H}$ NMR $\left(\mathrm{CDCl}_{3}\right) \delta 7.74(\mathrm{~d}, J=8.5,2 \mathrm{H}), 7.50(\mathrm{~d}, J=8.5,2 \mathrm{H}), 4.87(\mathrm{~s}, 2 \mathrm{H}) ;{ }^{13} \mathrm{C}$ NMR $\left(\mathrm{CDCl}_{3}\right) \delta 152.1(\mathrm{q}, J=1.6 \mathrm{~Hz}), 130.1(\mathrm{q}, J=33.1 \mathrm{~Hz}), 127.6(\mathrm{q}, J=3.7 \mathrm{~Hz}), 123.4(\mathrm{q}, J=272.3$ $\mathrm{Hz}$ ), 92.1, 80.5; ${ }^{19} \mathrm{~F}$ NMR $\left(\mathrm{CDCl}_{3}\right) \delta$-62.2; LRMS (EI), $\mathrm{m} / z$ (relative intensity), 372 (5), 300 (6), 254 (13), 241 (19), 174 (17), 162 (100), 131 (28); HRMS (EI) calcd. for $\mathrm{C}_{9} \mathrm{H}_{6} \mathrm{Cl}_{3} \mathrm{~F}_{3} \mathrm{O}_{4} \mathrm{~S}$ 371.9004 , found 371.8998 .

Sulfuric acid, 2,6-difluorophenyl ester 2,2,2-trichloroethyl ester (20). White solid; mp 33-34 ${ }^{\circ} \mathrm{C} ;{ }^{1} \mathrm{H}$ NMR $\left(\mathrm{CDCl}_{3}\right) \delta$ 7.26-7.37 (m, $\left.1 \mathrm{H}\right), 7.06(\mathrm{t}$ (overlapping dd), $J=8.3,2 \mathrm{H}), 4.97(\mathrm{~s}, 2 \mathrm{H})$; ${ }^{13} \mathrm{C}$ NMR $\left(\mathrm{CDCl}_{3}\right) \delta 155.4(\mathrm{dd}, J=254 \mathrm{~Hz}, J=2.9 \mathrm{~Hz}), 128.9(\mathrm{t}, J=9.3 \mathrm{~Hz}), 126.6(\mathrm{t}, J=15.6$ $\mathrm{Hz}), 112.9(\mathrm{dd}, J=16.2 \mathrm{~Hz}, J=3.0 \mathrm{~Hz})_{2} 92.3,80.9(\mathrm{t}, J=2.4 \mathrm{~Hz}) ;{ }^{19} \mathrm{~F} \mathrm{NMR}\left(\mathrm{CDCl}_{3}\right) \delta-124.4$; LRMS (EI), $m / z$ (relative intensity), 342.0 (13), 305.0 (7), 223 (13), 133.0 (14), 130.1 (100), 101.1 (25); HRMS (EI) calcd. for $\mathrm{C}_{8} \mathrm{H}_{5} \mathrm{Cl}_{3} \mathrm{~F}_{2} \mathrm{O}_{4} \mathrm{~S} 339.8942$, found 339.8936 .

Sulfuric acid, 4-methoxyphenyl ester 2,2,2-trichloroethyl ester (21). White solid; mp 62$64^{\circ} \mathrm{C} ;{ }^{1} \mathrm{H}$ NMR $\left(\mathrm{CDCl}_{3}\right) \delta 7.28(\mathrm{~d}, J=9.2 \mathrm{~Hz}, 2 \mathrm{H}), 6.92(\mathrm{~d}, J=9.2 \mathrm{~Hz}, 2 \mathrm{H}), 4.80(\mathrm{~s}, 2 \mathrm{H}), 3.82(\mathrm{~s}$, $3 \mathrm{H}) ;{ }^{13} \mathrm{C}$ NMR $\left(\mathrm{CDCl}_{3}\right) \delta 158.7,143.4,122.2,114.9,92.4,80.3,55.6$; LRMS (EI), $m / z$ (relative intensity), 334 (11), 216 (6), 203 (16), 123 (100), 95 (25); HRMS (EI) calcd. for $\mathrm{C}_{9} \mathrm{H}_{9} \mathrm{Cl}_{3} \mathrm{O}_{5} \mathrm{~S}$ 333.9236 , found 333.9232 .

Sulfuric acid 2-methylphenyl ester 2,2,2-trichloroethyl ester (22). White solid; mp $52-54{ }^{\circ} \mathrm{C}$; ${ }^{1} \mathrm{H}$ NMR $\left(\mathrm{CDCl}_{3}\right) \delta$ 7.33-7.38 (m, 1H), 7.20-7.29 (m, 3H), $4.81(\mathrm{~s}, 2 \mathrm{H}) 2.40(\mathrm{~s}, 3 \mathrm{H}) ;{ }^{13} \mathrm{C} \mathrm{NMR}$ $\left(\mathrm{CDCl}_{3}\right) \delta 149.0,132.1,131.0,128.0,127.5,121.1,92.6,80.4,16.4$; LRMS (EI), $\mathrm{m} / z$ (relative intensity), 320 (16), 201 (10), 188 (17), 108 (100); HRMS (EI) calcd. for $\mathrm{C}_{9} \mathrm{H}_{9} \mathrm{Cl}_{3} \mathrm{O}_{4} \mathrm{~S} 317.9287$, found 317.9281 .

Sulfuric acid, 4-iodophenyl ester 2,2,2-trichloroethyl ester (23). White solid; mp $45-47^{\circ} \mathrm{C} ;{ }^{1} \mathrm{H}$ NMR $\left(\mathrm{CDCl}_{3}\right) \delta 7.77(\mathrm{~d}, J=8.8 \mathrm{~Hz}, 2 \mathrm{H}), 7.12(\mathrm{~d}, J=8.8 \mathrm{~Hz}, 2 \mathrm{H}), 4.83(\mathrm{~s}, 2 \mathrm{H}) ;{ }^{13} \mathrm{C}$ NMR $\left(\mathrm{CDCl}_{3}\right) \delta 149.8,139.2,123.1,92.5,92.2,80.4$; LRMS (EI), $\mathrm{m} / z$ (relative intensity), 430 (25), 
299 (47); 219 (100), 130 (16),91 (29); HRMS (EI) calcd. for $\mathrm{C}_{8} \mathrm{H}_{6} \mathrm{IO}_{4} \mathrm{~S}$ 429.8097, found 429.8100 .

Sulfuric acid, 4-chlorophenyl ester 2,2,2-trichloroethyl ester (24). White solid; m.p. 27-28 ${ }^{\circ} \mathrm{C}$; ${ }^{1} \mathrm{H}$ NMR $\left(\mathrm{CDCl}_{3}\right) \delta 7.42(\mathrm{~d}, J=9.1 \mathrm{~Hz}, 2 \mathrm{H}), 7.31(\mathrm{~d}, J=9.1 \mathrm{~Hz}, 2 \mathrm{H}), 4.83(\mathrm{~s}, 2 \mathrm{H}) ;{ }^{13} \mathrm{C}$ NMR $\left(\mathrm{CDCl}_{3}\right) \delta 148.3,133.6,130.2,122.5,92.2,80.4,80.2$; LRMS (EI), $\mathrm{m} / z$ (relative intensity), 340 (13), 208 (25), 130 (41), 127 (100); HRMS (EI) calcd. for $\mathrm{C}_{8} \mathrm{H}_{6} \mathrm{Cl}_{4} \mathrm{O}_{4} \mathrm{~S}$ 337.8741, found 337.8733 .

Sulfuric acid, 4-acetylphenyl ester 2,2,2-trichloroethyl ester (25). White solid; m.p. $92-93{ }^{\circ} \mathrm{C}$; ${ }^{1} \mathrm{H} \mathrm{NMR}\left(\mathrm{CDCl}_{3}\right) \delta 8.06(\mathrm{~d}, J=9.1 \mathrm{~Hz}, 2 \mathrm{H}), 7.46(\mathrm{~d}, J=9.1 \mathrm{~Hz}, 2 \mathrm{H}), 4.86(\mathrm{~s}, 2 \mathrm{H}), 2.63(\mathrm{~s}, 3$ $\mathrm{H}) ;{ }^{13} \mathrm{C} \mathrm{NMR}\left(\mathrm{CDCl}_{3}\right) \delta 196.4,153.1,136.4,130.6,121.2,92.3,80.6,26.7$; LRMS (EI), $\mathrm{m} / \mathrm{z}$ (relative intensity), 348 (13), 332 (37), 201 (64), 132 (10) 121 (100), 107 (23); HRMS (EI) calcd. for $\mathrm{C}_{10} \mathrm{H}_{9} \mathrm{Cl}_{3} \mathrm{O}_{4} \mathrm{~S} 345.9236$, found 345.9232 .

Sulfuric acid, 3-estrone ester 2,2,2-trichloroethyl ester (26). White solid; m.p. 132-134 ${ }^{\circ} \mathrm{C} ;{ }^{1} \mathrm{H}$ NMR $\left(\mathrm{CDCl}_{3}\right) \delta 7.30(\mathrm{~d}, J=8.7 \mathrm{~Hz}, 1 \mathrm{H}), 7.05-7.10$ (overlapping d and s, $\left.3 \mathrm{H}\right), 4.80(\mathrm{~s}, 2 \mathrm{H}), 2.9$ (bs, 2H), 1.93-2.53 (m, 7H), 1.37-1.67 (m, 6H), $0.89(\mathrm{~s}, 3 \mathrm{H}) ;{ }^{13} \mathrm{C} \mathrm{NMR}\left(\mathrm{CDCl}_{3}\right) \delta 220.6,148.1$, 139.8, 139.1, 127.1, 121.1, 118.2, 92.5, 80.4, 50.4, 47.9, 44.2, 37.9, 35.9, 31.6, 29.5, 26.2, 25.8, 21.6, 13.9; LRMS (EI), $m / z$ (relative intensity), 4809 (98), 438 (45), 423 (41), 253 (63), 213 (95), 97 (100); HRMS (EI) calcd. for $\mathrm{C}_{20} \mathrm{H}_{25} \mathrm{Cl}_{3} \mathrm{O}_{5} \mathrm{~S}, 480.00332$, found 480.0333 .

4-(2,2,2-Trichloro-ethoxysulfonyloxy)-benzoic acid benzyl ester (39). Colorless oil; ${ }^{1} \mathrm{H}$ NMR $\left(\mathrm{CDCl}_{3}\right) \delta 8.15(\mathrm{~d}, J=8.8 \mathrm{~Hz}), 7.32-7.49(\mathrm{~m}, 7 \mathrm{H}), 5.36(\mathrm{~s}, 2 \mathrm{H}), 4.83(\mathrm{~s}, 2 \mathrm{H}) ;{ }^{13} \mathrm{C}$ NMR $\left(\mathrm{CDCl}_{3}\right)$ $\delta 165.04,153.3,135.7,132.0,129.8,128.8,128.6,128.4,121.1,92.3,80.6,67.3$; LRMS (EI), $\mathrm{m} / z$ (relative intensity), 440 (11), 335 (21), 331 (45), 201 (32), 121 (34), 91 (100); HRMS (EI) calcd. for $\mathrm{C}_{16} \mathrm{H}_{13} \mathrm{Cl}_{3} \mathrm{O}_{6} \mathrm{~S} 437.9498$, found 437.9499 .

General procedure for deprotection of TCE sulfate esters 16-26 using Pd/C-ammonium formate. To a solution of the trichloroethyl ester (16-25, $200 \mathrm{mg}$ ) in HPLC grade $\mathrm{MeOH}(3.5$ $\mathrm{mL}$ ) was added ammonium formate (6 equiv) and $10 \mathrm{wt}$ \% of $10 \% \mathrm{Pd} / \mathrm{C}$. Estrone derivative $\mathbf{2 6}$ does not dissolve well in MeOH. Thus, compound 26 (200 mg) was dissolved in THF (3 mL) and then $\mathrm{MeOH}(3 \mathrm{~mL})$ was added followed by the addition of ammonium formate (6 equiv) and 10 wt. $\%$ of $10 \% \mathrm{Pd} / \mathrm{C}$. The solution was stirred until all of the ester was consumed as determined by TLC (usually within $8 \mathrm{~h}$ ). The Pd catalyst was removed by centrifugation or by filtration through Celite and the supernatant was concentrated in vacuo to give a white residue. This solid was dissolved in a minimal $\mathrm{CH}_{2} \mathrm{Cl}_{2} / \mathrm{MeOH} / \mathrm{NH}_{4} \mathrm{OH}$ (5:4:0.5) and purified by flash chromatography using $\mathrm{CH}_{2} \mathrm{Cl}_{2} / \mathrm{MeOH} / \mathrm{NH}_{4} \mathrm{OH}$ (10:2:0.5) as eluant. Fractions containing the desired product were pooled, concentrated and the residue dissolved in water and lyophilized. The lyophilization procedure was repeated. This gave compounds 27-37 as white, fluffy ammonium salts. See Table 1 in main manuscript for yields.

General procedure for deprotection of TCE sulfate esters 16-26 using Zn-ammonium formate. To a solution of the trichloroethyl ester (16-25, $200 \mathrm{mg})$ in HPLC grade $\mathrm{MeOH}(3.5$ $\mathrm{mL}$ ) was added ammonium formate (6 equiv) and $\mathrm{Zn}$ dust ( 2 equiv). Estrone derivative $\mathbf{2 6}$ does 
not dissolve well in MeOH. Thus, compound 26 (200 mg) was dissolved in THF (3 mL) and then $\mathrm{MeOH}(3 \mathrm{~mL}$ ) was added followed by the addition of ammonium formate ( 6 equiv) and $\mathrm{Zn}$ dust ( 2 equiv). The solution was stirred until all of the ester was consumed as determined by TLC (usually within $2 \mathrm{~h}$ ). The reaction was filtered through Celite and the supernatant was concentrated in vacuo to give a white residue. This solid was dissolved in a minimal $\mathrm{CH}_{2} \mathrm{Cl}_{2} / \mathrm{MeOH} / \mathrm{NH}_{4} \mathrm{OH} \quad(5: 4: 0.5)$ and purified by flash chromatography using $\mathrm{CH}_{2} \mathrm{Cl}_{2} / \mathrm{MeOH} / \mathrm{NH}_{4} \mathrm{OH}(10: 2: 0.5)$ as eluant. Fractions containing the desired product were pooled, concentrated and the residue dissolved in water and lyophilized. The lyophilization procedure was repeated. This gave compounds 27-37 as white, fluffy ammonium salts. See Table 1 in main manuscript for yields. Spectral data for all compounds prepared in this manner were identical to the spectral data obtained using the Pd-ammonium formate method.

4-fluorophenylsulfate, ammonium salt (27). ${ }^{2} \quad{ }^{1} \mathrm{H}$ NMR $\left(\mathrm{D}_{2} \mathrm{O}\right) \delta$ 7.19-7.31 (m, 2H), 7.15 (overlapping dd, J = 8.8 Hz, 2H) $\left(4.83(\mathrm{~s}, 2 \mathrm{H}) ;{ }^{19} \mathrm{~F}\right.$ NMR $\left(\mathrm{D}_{2} \mathrm{O}\right) \delta-120.4 ;{ }^{13} \mathrm{C} \mathrm{NMR}\left(\mathrm{D}_{2} \mathrm{O}\right) \delta$ 160.4 (d, J = $242 \mathrm{~Hz}), 147.0$ (d, J = 2.4 Hz), 123.3 (d, J = 8.5 Hz), 116.2 (d, J = 23.8 Hz); LRMS (ESI) $m / z$ (relative intensity) $191\left(\mathrm{M}-\mathrm{NH}_{4}{ }^{+}, 100\right), 111\left(\mathrm{M}-\mathrm{SO}_{3} \mathrm{NH}_{4}, 13\right)$; HRMS (ESI) calcd. for $\mathrm{C}_{6} \mathrm{H}_{4} \mathrm{FO}_{6} \mathrm{~S} 190.9819$, found 190.9813 .

phenylsulfate, ammonium salt (28). ${ }^{3}{ }^{1} \mathrm{H}$ NMR $\left(\mathrm{D}_{2} \mathrm{O}\right) \delta 7.43$ (overlapping dd, $J=7.9 \mathrm{~Hz}, 2 \mathrm{H}$ ), $7.30(\mathrm{t}, J=7.5 \mathrm{~Hz}, 3 \mathrm{H}) ;{ }^{13} \mathrm{C} \mathrm{NMR}\left(\mathrm{D}_{2} \mathrm{O}\right) \delta 151.1,130.0,126.5,121.6$; LRMS (ESI): $\mathrm{m} / z$ (relative intensity) $173.0\left(\mathrm{M}-\mathrm{NH}_{4}^{+}, 100\right)$; HRMS (ESI) calcd. for $\mathrm{C}_{6} \mathrm{H}_{5} \mathrm{O}_{4} \mathrm{~S} 172.9914$, found 172.9911 .

4-nitrophenylsulfate, ammonium salt (29). ${ }^{4} \mathrm{H}$ NMR $\left(\mathrm{D}_{2} \mathrm{O}\right) \delta 8.31(\mathrm{~d}, J=9.2 \mathrm{~Hz}, 2 \mathrm{H}), 7.48(\mathrm{~d}$, $J=7.9 \mathrm{~Hz}, 2 \mathrm{H}) ;{ }^{13} \mathrm{C}$ NMR $\left(\mathrm{D}_{2} \mathrm{O}\right) \delta 156.4,144.5,125.6,121.4$; LRMS (ESI) $\mathrm{m} / z$ (relative intensity) $218\left(\mathrm{M}-\mathrm{NH}_{4}^{+}, 100\right), 138\left(\mathrm{M}-\mathrm{SO}_{3} \mathrm{NH}_{4}, 68\right)$. HRMS (ESI) calcd. for $\mathrm{C}_{6} \mathrm{H}_{4} \mathrm{NO}_{6} \mathrm{~S}$ 217.9764, found 217.9758.

4-trifluoromethylphenylsulfate, ammonium salt (30). ${ }^{5}{ }^{1} \mathrm{H} \mathrm{NMR}\left(\mathrm{D}_{2} \mathrm{O}\right) \delta 7.74(\mathrm{~d}, J=8.0 \mathrm{~Hz}$, $2 \mathrm{H}), 7.43(\mathrm{~d}, J=8.0 \mathrm{~Hz}, 2 \mathrm{H}) ;{ }^{13} \mathrm{C} \operatorname{NMR}\left(\mathrm{D}_{2} \mathrm{O}\right) \delta 153.8(\mathrm{q}, J=1.5 \mathrm{~Hz}), 127.2(\mathrm{q}, J=32.5 \mathrm{~Hz})$, $127.0(\mathrm{q}, J=3.9 \mathrm{~Hz}), 124.0(\mathrm{q}, J=271.3 \mathrm{~Hz}), 122.2,121.6 ;{ }^{19} \mathrm{~F}$ NMR $\left(\mathrm{D}_{2} \mathrm{O}\right) \delta-61.7$; LRMS (ESI) $m / z$ (relative intensity), $241\left(\mathrm{M}-\mathrm{NH}_{4}^{+}, 100\right), 161\left(\mathrm{M}-\mathrm{SO}_{3} \mathrm{NH}_{4}, 11\right)$; HRMS (ESI) calcd. for $\mathrm{C}_{7} \mathrm{H}_{4} \mathrm{~F}_{3} \mathrm{O}_{4} \mathrm{~S} 240.9787$, found 240.9787 .

2,6-difluorophenylsulfate, ammonium salt (31). ${ }^{6}{ }^{1} \mathrm{H}$ NMR $\left(\mathrm{D}_{2} \mathrm{O}\right) \delta 6.98-7.21(\mathrm{~m}, 3 \mathrm{H}),{ }^{13} \mathrm{C}$ $\operatorname{NMR}\left(\mathrm{D}_{2} \mathrm{O}\right) \delta 155.93(\mathrm{dd}, J=249.6 \mathrm{~Hz}, \mathrm{~J}=3.5 \mathrm{~Hz}), 127.5(\mathrm{t}, J=15.7 \mathrm{~Hz}), 127.2(\mathrm{t}, J=9.1 \mathrm{~Hz})$, $112.5(\mathrm{dd}, J=18.8 \mathrm{~Hz}, \mathrm{~J}=4.2 \mathrm{~Hz}) ;{ }^{19} \mathrm{~F} \mathrm{NMR}\left(\mathrm{D}_{2} \mathrm{O}\right) \delta-126.7 \mathrm{LRMS}$ (ESI) $\mathrm{m} / z$ (relative intensity), $209\left(\mathrm{M}-\mathrm{NH}_{4}^{+}, 100\right), 129$ (M - $\left.\mathrm{SO}_{3} \mathrm{NH}_{4}, 45\right)$; HRMS (ESI) calcd. for $\mathrm{C}_{6} \mathrm{H}_{3} \mathrm{~F}_{2} \mathrm{O}_{4} \mathrm{~S}$ 208.9725, found 208.9722.

4-methoxyphenylsulfate, ammonium salt (32). ${ }^{7} \mathrm{H}$ NMR $\left(\mathrm{D}_{2} \mathrm{O}\right) \delta 7.41(\mathrm{~d}, J=8.8 \mathrm{~Hz}, 2 \mathrm{H})$, $7.25(\mathrm{~d}, J=8.8 \mathrm{~Hz}, 2 \mathrm{H}) ;{ }^{13} \mathrm{C}$ NMR $\left(\mathrm{D}_{2} \mathrm{O}\right) \delta 149.7,131.0,129.7$, 123.1; LRMS (ESI) $\mathrm{m} / z$ (relative intensity), $203\left(\mathrm{M}-\mathrm{NH}_{4}^{+}, 100\right), 188\left(\mathrm{M}-\mathrm{CH}_{3}-\mathrm{NH}_{4}{ }^{+}, 7\right), 108\left(\mathrm{M}-\mathrm{CH}_{3}-\mathrm{SO}_{3} \mathrm{NH}_{4}, 4 \%\right)$; HRMS (ESI) calcd. for $\mathrm{C}_{7} \mathrm{H}_{7} \mathrm{O}_{5} \mathrm{~S}, 203.0019$, found 203.0024. 
2-methylphenylsulfate, ammonium salt (33). ${ }^{8}{ }^{1} \mathrm{H}$ NMR $\left(\mathrm{D}_{2} \mathrm{O}\right) \delta 7.0-7.25(\mathrm{~m}, 4 \mathrm{H}), 2.19(\mathrm{~s}, 3 \mathrm{H})$;

${ }^{13} \mathrm{C}$ NMR $\left(\mathrm{D}_{2} \mathrm{O}\right) \delta 149.6,131,5,127.1,126.4,121.5,15.6$; LRMS (ESI) $m / z$ (relative intensity), $187\left(\mathrm{M}-\mathrm{NH}_{4}{ }^{+}, 100\right), 107\left(\mathrm{M}-\mathrm{SO}_{3} \mathrm{NH}_{4}, 12\right)$; HRMS (ESI) calcd. for $\mathrm{C}_{6} \mathrm{H}_{7} \mathrm{O}_{4} \mathrm{~S}, 187.0070$, found 187.0072 .

4-iodophenylsulfate, ammonium salt (34). ${ }^{1} \mathrm{H}$ NMR $\left(\mathrm{D}_{2} \mathrm{O}\right) \delta 7.78(\mathrm{~d}, J=8.8 \mathrm{~Hz}, 2 \mathrm{H}), 7.09$ (d, $J=8.8 \mathrm{~Hz}, 2 \mathrm{H}) ;{ }^{13} \mathrm{C}$ NMR $\left(\mathrm{D}_{2} \mathrm{O}\right) \delta 151.1$, 138.8, 123.7, 90.0; LRMS (ESI) $\mathrm{m} / z$ (relative intensity), $298\left(\mathrm{M}-\mathrm{NH}_{4}^{+}, 100\right), 219\left(\mathrm{M}-\mathrm{SO}_{3} \mathrm{NH}_{4}, 10\right)$; HRMS (ESI) calcd. for $\mathrm{C}_{6} \mathrm{H}_{4} \mathrm{IO}_{4} \mathrm{~S}$, 298.8880 , found 298.8875 .

4-chlorophenylsulfate, ammonium salt (35). ${ }^{9} \mathrm{H}$ NMR $\left(\mathrm{D}_{2} \mathrm{O}\right) \delta 7.41(\mathrm{~d}, J=8.8 \mathrm{~Hz}, 2 \mathrm{H}), 7.25$ $(\mathrm{d}, J=8.8 \mathrm{~Hz}, 2 \mathrm{H}) ;{ }^{13} \mathrm{C} \mathrm{NMR}\left(\mathrm{D}_{2} \mathrm{O}\right) \delta 149.7,131.0,129.7,123.1$; LRMS (ESI) $\mathrm{m} / z$ (relative intensity), $209\left(\mathrm{M}-\mathrm{NH}_{4}{ }^{+}, 45\right), 207\left(\mathrm{M}-\mathrm{NH}_{4}{ }^{+}, 100\right), 127$ (M - $\left.\mathrm{SO}_{3} \mathrm{NH}_{4}, 15 \%\right)$; HRMS (ESI) calcd. for $\mathrm{C}_{6} \mathrm{H}_{4} \mathrm{ClO}_{4} \mathrm{~S}, 206.9524$, found 206.9523 .

4-acetylphenylsulfate, ammonium salt (36). ${ }^{10}{ }^{1} \mathrm{H}$ NMR $\left(\mathrm{D}_{2} \mathrm{O}\right) \delta 8.02(\mathrm{~d}, J=8.4 \mathrm{~Hz}, 2 \mathrm{H}), 7.40$ $(\mathrm{d}, J=8.4 \mathrm{~Hz} 2 \mathrm{H}), 2.64(\mathrm{~s}, 3 \mathrm{H}) ;{ }^{13} \mathrm{C} \mathrm{NMR}\left(\mathrm{D}_{2} \mathrm{O}\right) \delta 202.3,155.5,133.6,130.6,121.0,26.2$; LRMS (ESI) $m / z$ (relative intensity), $215\left(\mathrm{M} \mathrm{-} \mathrm{NH}_{4}{ }^{+}, 100\right), 135\left(\mathrm{M}-\mathrm{SO}_{3} \mathrm{NH}_{4}, 30\right)$; HRMS (ESI) calcd. for $\mathrm{C}_{8} \mathrm{H}_{7} \mathrm{O}_{5} \mathrm{~S}, 215.0019$, found 215.0020.

Estrone-3-sulfate (37). ${ }^{11}{ }^{1} \mathrm{H}$ NMR $\left(\mathrm{D}_{2} \mathrm{O}\right) \delta 7.18(\mathrm{~d}, J=8.6 \mathrm{~Hz}, 1 \mathrm{H}), 7.00(\mathrm{~d}, J=8.6 \mathrm{~Hz}, 1 \mathrm{H})$, $6.99(\mathrm{~s}, 1 \mathrm{H}), 2.82(\mathrm{~m}, 2 \mathrm{H}), 2.3-2.54(\mathrm{~m}, 2 \mathrm{H}), 1.76-2.23(\mathrm{~m}, 5 \mathrm{H}), 1.26-1.70(\mathrm{~m}, 6 \mathrm{H}), 0.85(\mathrm{~s}, 3 \mathrm{H})$; ${ }^{13} \mathrm{C}$ NMR $\left(\mathrm{D}_{2} \mathrm{O}\right) \delta 222.7,150.4,137.5,136.4,125.8,121.2,118.5,50.2,47.9,44.0,38.2,35.5$, 31.4, 29.2, 26.2, 25.7, 21.2, 13.1.

4-sulfooxybenzoic acid (40). ${ }^{12}$ Compound 39 (157 mg, $\left.0.357 \mathrm{mmol}\right)$ was dissolved in HPLC grade $\mathrm{MeOH}(7 \mathrm{~mL})$. To this was added ammonium formate $(270 \mathrm{mg}, 12$ equiv) and $10 \% \mathrm{Pd} / \mathrm{C}$ (30 mg, $20 \mathrm{wt}$ \%). The solution was stirred for $36 \mathrm{~h}$ then filtered through Celite and the Celite was washed with $\mathrm{MeOH}(20 \mathrm{~mL})$. The filtrate was concentrated leaving a white solid. This was dissolved in a minimal amount of methanol and subjected to silica gel chromatography $\left(\mathrm{CH}_{2} \mathrm{Cl}_{2} / \mathrm{MeOH} / \mathrm{NH}_{4} \mathrm{OH}, 8.5: 2: 5: 1\right)$ which gave pure 40 as a white solid in an $81 \%$ yield. ${ }^{1} \mathrm{H}$ $\operatorname{NMR}\left(\mathrm{D}_{2} \mathrm{O}\right) \delta 7.81(\mathrm{~d}, J=6.4 \mathrm{~Hz}, 2 \mathrm{H}), 7.25(\mathrm{~d}, J=6.4 \mathrm{~Hz}, 2 \mathrm{H}) ;{ }^{13} \mathrm{C} \mathrm{NMR}\left(\mathrm{D}_{2} \mathrm{O}\right) \delta 174.7,153.2$, 133.8, 130.5, 120.9; LRMS (ESI) $\mathrm{m} / z$ (relative intensity), 217, (M - $\left.\mathrm{NH}_{4}^{+}, 100\right), 137(\mathrm{M} \mathrm{-}$ $\mathrm{SO}_{3} \mathrm{NH}_{4}, 23$ );HRMS (ESI) calcd. for $\mathrm{C}_{7} \mathrm{H}_{5} \mathrm{O}_{6} \mathrm{~S}, 216.9812$, found 216.9816 .

Sulfuric acid, 4-carboxyphenyl ester, 2,2,2-trichloroethyl ester, ammonium salt (41). Compound 39 (185 mg, $0.421 \mathrm{mmol})$ was dissolved in TFA/AcOH (1:1, $2 \mathrm{~mL}) . \quad 10 \% \mathrm{Pd} / \mathrm{C}(9.4$ mg, 5 wt. \%) was added and the solution subjected to $\mathrm{H}_{2}$ (balloon pressure). After $3.5 \mathrm{~h}$, the reaction was filtered through Celite and the Celite washed with $\mathrm{AcOH}(10 \mathrm{~mL})$. The filtrate was concentrated by high vacuum rotary evaporation to give a white residue. Flash chromatography $\left(\mathrm{CH}_{2} \mathrm{Cl}_{2} / \mathrm{MeOH} / \mathrm{NH}_{4} \mathrm{OH}, 10: 2: 0.5\right)$ of the residue gave 41 as a white solid in $82 \%$ yield. It was found that longer reaction times resulted in reduced yields due to loss of the TCE group and desulfation. ${ }^{1} \mathrm{H}$ NMR $\left(\mathrm{CD}_{3} \mathrm{OD}\right) \delta 8.05(\mathrm{~d}, J=8.5 \mathrm{~Hz}, 2 \mathrm{H}), 7.41(\mathrm{~d}, J=8.5 \mathrm{~Hz}, 2 \mathrm{H}), 5.09$ (s, 2H); 
${ }^{13} \mathrm{C}$ NMR $\left(\mathrm{CD}_{3} \mathrm{OD}\right) \delta 169.1,150.3,134.0,129.4,118.8,91.0,78.7$; LRMS (ESI) $\mathrm{m} / z$ (relative intensity), 349 (M - $\left.\mathrm{NH}_{4}{ }^{+}, 100\right)$; HRMS (EI) calcd. for $\mathrm{C}_{14} \mathrm{H}_{11} \mathrm{O}_{6} \mathrm{~S} 346.8897$, found 346.8885 .

4-Sulfooxybenzoic acid benzyl ester (42). Prepared as white fluffy solid in 95\% yield from 39 using the Zn-ammonium formate procedure described above. ${ }^{1} \mathrm{H}$ NMR $\left(\mathrm{D}_{2} \mathrm{O}\right) \delta 7.92(\mathrm{~d}, J=8.5$ $\mathrm{Hz}, 2 \mathrm{H}), 7.23-7.39(\mathrm{~m}, 7 \mathrm{H}), 5.24(\mathrm{~s}, 2 \mathrm{H}) ;{ }^{13} \mathrm{C} \mathrm{NMR}\left(\mathrm{D}_{2} \mathrm{O}\right) \delta 166.6,155.3,135.4,131.1,128.4$, 128.1, 127.9, 126.2, 120.8, 66.7; LRMS (ESI) $\mathrm{m} / z$ (relative intensity), $307\left(\mathrm{M} \mathrm{-} \mathrm{NH}_{4}{ }^{+}, 100\right), 227$ $\left(\mathrm{M}-\mathrm{SO}_{3} \mathrm{NH}_{4}, 10\right)$; HRMS (ESI) calcd. for $\mathrm{C}_{14} \mathrm{H}_{11} \mathrm{O}_{6} \mathrm{~S}, 307.0281$, found 307.0284.

3-Hydroxylestra-1,3,5(10)-triene-17-one-2-carboxaldehyde $(43) .{ }^{13}$ To a mixture of estrone (1.35 g, $5.0 \mathrm{mmol})$, paraformaldehyde $(1.50 \mathrm{~g}, 50 \mathrm{mmol})$ and magnesium chloride $(1.25 \mathrm{~g}, 13$ $\mathrm{mmol})$ in a glass bomb was added acetonitrile $(20 \mathrm{~mL})$, followed by tributylamine $(5.5 \mathrm{~mL}, 23$ $\mathrm{mmol})$ at room temperature. The sealed tube was stirred at room temperature for 2 minutes and then heated at $125^{\circ} \mathrm{C}$ for $11 \mathrm{~h} .6 \mathrm{~N} \mathrm{HCl}(15 \mathrm{~mL})$ was added slowly after cooling and the mixture extracted with diethyl ether $(100 \mathrm{~mL}, 2 \times 50 \mathrm{~mL})$. The extracts were combined, washed with $\mathrm{H}_{2} \mathrm{O}(2 \times 50 \mathrm{~mL})$, brine $(50 \mathrm{~mL})$, then dried over $\mathrm{Na}_{2} \mathrm{SO}_{4} \cdot{ }^{1} \mathrm{H} \mathrm{NMR}$ of the crude material revealed that $\mathbf{4 3}$ and 4-formyl estrone were formed in a ratio of 14:1. Column chromatography (silica, 2:1 hexane/ethyl acetate) gave $\mathbf{4 3}$ as colorless solid (768 mg, $2.58 \mathrm{mmol}, 52 \%$ yield) and a mixture of 43 and 4-formyl estrone $\left(184 \mathrm{mg}, 0.62 \mathrm{mmol}, 12 \%\right.$ yield). ${ }^{1} \mathrm{H} \mathrm{NMR}\left(\mathrm{CDCl}_{3}\right) \delta$ $10.65(\mathrm{~s}, 1 \mathrm{H}), 9.65(\mathrm{~s}, 1 \mathrm{H}), 7.29(\mathrm{~s}, 1 \mathrm{H}), 6.53(\mathrm{~s}, 1 \mathrm{H}), 1.70-1.90,(\mathrm{~m}, 2 \mathrm{H}), 1.77-2.41(\mathrm{~m}, 7 \mathrm{H})$, $1.22-1.60(\mathrm{~m}, 6 \mathrm{H}), 0.80(\mathrm{~s}, 3 \mathrm{H})$.

2-Formylestra-1,3,5(10)-triene-17-one-3-(2,2,2-trichloroethyl) sulfate (44). To a solution of 43 (1.38 g, $4.63 \mathrm{mmol})$ and DMAP (534 mg, $4.45 \mathrm{mmol})$ in dry THF (50 mL) was added triethylamine $(1.3 \mathrm{~mL}, 9.34 \mathrm{mmol})$ followed by chlorosulfuric acid 2,2,2-trichloroethyl ester (4, $1.2 \mathrm{~mL}, 9.00 \mathrm{mmol})$. The mixture was then stirred for $14 \mathrm{~h}$ at room temperature. The reaction mixture was diluted with ethyl acetate $(100 \mathrm{~mL})$ and $\mathrm{H}_{2} \mathrm{O}(40 \mathrm{~mL})$. After separation, the aqueous phase was extracted with ethyl acetate $(2 \times 50 \mathrm{~mL})$. The combined organic layer was washed with $0.5 \mathrm{~N} \mathrm{HCl}(2 \times 40 \mathrm{~mL})$, water $(40 \mathrm{~mL})$ and brine $(40 \mathrm{~mL})$, then dried $\left(\mathrm{Na}_{2} \mathrm{SO}_{4}\right)$. Column chromatography $(2: 1$ hexane/ethyl acetate) yielded 44 as colorless oil, that solidified on standing, in 97\% yield. ${ }^{1} \mathrm{H}$ NMR $\left(\mathrm{CDCl}_{3}\right) \delta 10.21(\mathrm{~s}, 1 \mathrm{H}), 7.85(\mathrm{~s}, 1 \mathrm{H}), 7.24(\mathrm{~s}, 1 \mathrm{H}), 4.92(\mathrm{~s}, 2 \mathrm{H})$, 2.85-3.04 (m, 2H), 1.91-2.60 (m, 7H), 1.40-1.75 (m, 6H), $0.90(\mathrm{~s}, 3 \mathrm{H}) ;{ }^{13} \mathrm{C} \mathrm{NMR}\left(\mathrm{CDCl}_{3}\right) \delta$ 220.2, 187.4, 148.4, 146.4, 140.7, 128.1, 125.8, 122.4, 92.4, 80.7, 50.3, 47.8, 43.9, 37.6, 35.8, 31.4, 29.9, 25.8, 25.7, 21.6, 13.8; LRMS (EI): $\mathrm{m} / z$ (relative intensity), $510(\mathrm{M}+2,93), 508\left(\mathrm{M}^{+}\right.$, 94), 466 (74), 464 (70), 451 (44), 361 (56), 298 (100), 241 (89); HRMS (EI) calcd. for $\mathrm{C}_{21} \mathrm{H}_{23} \mathrm{Cl}_{3} \mathrm{O}_{6} \mathrm{~S} 508.0281$, found 508.0291.

2-Difluoromethylestra-1,3,5(10)-triene-17-one-3-(2,2,2-trichloroethyl) sulfate (45). To a solution of $44(385 \mathrm{mg}, 0.756 \mathrm{mmol})$ in dry methylene chloride $(10 \mathrm{~mL})$ at $0^{\circ} \mathrm{C}$ was added DAST (diethylaminosulfur trifluoride, $0.3 \mathrm{~mL}, 2.27 \mathrm{mmol}$ ) via syringe. The pale orange mixture was stirred for $1 \mathrm{~h}$ at that temperature, then overnight at room temperature. After slowly quenching with saturated $\mathrm{NaHCO}_{3}(7 \mathrm{~mL})$, the reaction mixture was stirred for additional 10 min and extracted with ethyl acetate $(3 \times 30 \mathrm{~mL})$. The combined organic extracts were washed successively with water $(2 \times 20 \mathrm{~mL})$ and brine $(20 \mathrm{~mL})$ and dried $\left(\mathrm{Na}_{2} \mathrm{SO}_{4}\right)$. Column 
chromatography (silica, 5:2 hexane/ethyl acetate) yielded $\mathbf{4 5}$ as white foam in $91 \%$ yield. ${ }^{1} \mathrm{H}$ NMR $\left(\mathrm{CDCl}_{3}\right) \delta 7.52(\mathrm{~s}, 1 \mathrm{H}), 7.20(\mathrm{~s}, 1 \mathrm{H}), 6.82(\mathrm{t}, 1 \mathrm{H}, J=55 \mathrm{~Hz}), 4.82(\mathrm{~s}, 2 \mathrm{H}), 2.90-3.0(\mathrm{bs}$, 2H), 1.90-2.51 (m, 7H), 1.31-1.67 (m, 6H), $0.84(\mathrm{~s}, 3 \mathrm{H}) ;{ }^{13} \mathrm{C} \mathrm{NMR}\left(\mathrm{CDCl}_{3}\right) \delta 220.3,145.4(\mathrm{t}, J=$ $5 \mathrm{~Hz}), 142.3(\mathrm{t}, J=2 \mathrm{~Hz}), 140.3,124.5(\mathrm{t}, J=6 \mathrm{~Hz}), 123.6(\mathrm{t}, J=23 \mathrm{~Hz}), 121.3,111.3(\mathrm{t}, J=237$ $\mathrm{Hz}$ ), 92.4, 80.6, 50.3, 47.8, 44.0, 37.6, 35.8, 31.4, 29.5, 25.9, 25.7, 21.6, 13.8; ${ }^{19} \mathrm{~F}$ NMR (proton coupled, $\left.\mathrm{CDCl}_{3}\right) \delta-111.8(\mathrm{dd}, J=304.4 \mathrm{~Hz}, 54.7 \mathrm{~Hz}),-113.0(\mathrm{dd}, J=304.4 \mathrm{~Hz}, 54.7 \mathrm{~Hz})$; LRMS (EI): $m / z$ (relative intensity), $532(\mathrm{M}+2,60), 530\left(\mathrm{M}^{+}, 60\right), 488(46), 486(44), 475$ (34), 473 (30), 301 (47), 263 (66), 97 (100); HRMS (EI) calcd. for $\mathrm{C}_{21} \mathrm{H}_{23} \mathrm{Cl}_{3} \mathrm{~F}_{2} \mathrm{O}_{5} \mathrm{~S} 530.0300$, found 530.0308 .

Ammonium 2-difluoromethyl-3-(sulfonatooxy)estra-1,3,5(10)-triene-17-one (1). To a solution of the ester 45 (498 mg, $0.94 \mathrm{mmol}$ ) in HPLC grade $\mathrm{MeOH}$ (12 mL) was added ammonium formate $(370 \mathrm{mg}, 5.87 \mathrm{mmol})$ and $75 \mathrm{mg}(15 \mathrm{wt}$ \% $)$ of $10 \% \mathrm{Pd} / \mathrm{C}$. The reaction mixture was stirred for $24 \mathrm{~h}$. The Pd catalyst was removed by filtration and the filtrate was concentrated in vacuo to give a white residue. The residue was dissolved in water and lyophilized to give a white fluffy solid. This solid was dissolved in a minimal $\mathrm{CH}_{2} \mathrm{Cl}_{2} / \mathrm{MeOH} / \mathrm{NH}_{4} \mathrm{OH} \quad(5: 4: 0.5)$ and purified by flash chromatography using $\mathrm{CH}_{2} \mathrm{Cl}_{2} / \mathrm{MeOH} / \mathrm{NH}_{4} \mathrm{OH}$ (10:2:0.5) as eluant. Fractions containing 1 were pooled, concentrated and the residue dissolved in water and lyophilized. The lyophilization procedure was repeated. This gave compound $\mathbf{1}$ as a white fluffy ammonium salt in $86 \%$ yield. ${ }^{1} \mathrm{H}$ NMR $\left(\mathrm{CD}_{3} \mathrm{OD}\right) \delta$ $7.47(\mathrm{~s}, 1 \mathrm{H}), 7.20(\mathrm{~s}, 1 \mathrm{H}), 7.03(\mathrm{t}, 1 \mathrm{H}, J=56 \mathrm{~Hz}), 4.86(\mathrm{~s}, 4 \mathrm{H}), 2.85-3.00(\mathrm{bs}, 2 \mathrm{H}), 1.83-2.60$ (m, $7 \mathrm{H}), 1.40-1.80(\mathrm{~m}, 6 \mathrm{H}), 0.88(\mathrm{~s}, 3 \mathrm{H}) ;{ }^{13} \mathrm{C} \mathrm{NMR}\left(\mathrm{CD}_{3} \mathrm{OD}\right) \delta 222.5,148.0(\mathrm{t}, J=7 \mathrm{~Hz}), 140.8(\mathrm{t}, J$ $=2 \mathrm{~Hz}), 137.2,124.5(\mathrm{t}, J=23 \mathrm{~Hz}), 122.3(\mathrm{t}, J=5 \mathrm{~Hz}), 122.2,112.0(\mathrm{t}, J=233 \mathrm{~Hz}), 50.2,47.9$, $43.9,38.0,35.4,31.3,29.1,25.9,25.6,21.2,12.9 ;{ }^{19} \mathrm{~F}$ NMR (proton coupled, $\mathrm{CD}_{3} \mathrm{OD}$ ) $\delta-113.8$ (d, $J=55.6 \mathrm{~Hz}$ ); LRMS (ESI) $m / z$ (relative intensity) 399.1 (M - $\mathrm{NH}_{4}^{+}, 100$ ); HRMS (ESI) calcd. for $\mathrm{C}_{19} \mathrm{H}_{21} \mathrm{O}_{5} \mathrm{~F}_{2} \mathrm{~S} 399.1083$, found 399.1096.

2-Hydroxymethylestra-1,3,5(10)-triene-17-one-3-(2,2,2-trichloroethyl) sulfate (46).

A mixture of $\mathrm{Zr}\left(\mathrm{O}^{\mathrm{i}} \mathrm{Pr}\right){ }_{4} \cdot{ }^{\mathrm{i}} \mathrm{PrOH}(1.34 \mathrm{~g}, 3.46 \mathrm{mmol})$ and $( \pm)$-BINOL (981 $\left.\mathrm{mg}, 3.43 \mathrm{mmol}\right)$ in toluene $(12 \mathrm{~mL})$ was heated at $60^{\circ} \mathrm{C}$ for $65 \mathrm{~min}$. After cooling down to room temperature, a solution of $45(1.81 \mathrm{~g}, 3.55 \mathrm{mmol})$ in toluene $(5 \mathrm{~mL})$ was added. The reaction mixture was stirred for $30 \mathrm{~min}$ at room temperature. The reaction was quenched with saturated $\mathrm{NaHCO}_{3}(30$ $\mathrm{mL})$ and extracted with ethyl acetate $(3 \times 100 \mathrm{~mL})$. The combined organic extracts were washed successively with water $(2 \times 100 \mathrm{~mL})$ and brine $(100 \mathrm{~mL})$ and dried $\left(\mathrm{Na}_{2} \mathrm{SO}_{4}\right)$. Column chromatography (silica, 3:2 hexane/ethyl acetate) yielded 46 as white foam in $95 \%$ yield. ${ }^{1} \mathrm{H}$ NMR $\left(\mathrm{CDCl}_{3}\right) \delta 7.44$ (s, 1H), 7.12 (s. 1H), 4.88 (s, 2H), 4.72 (s, 2H), 2.87-2.97 (m, 2H), 1.90$2.60(\mathrm{~m}, 7 \mathrm{H}), 1.34-1.73(\mathrm{~m}, 7 \mathrm{H}), 0.90(3 \mathrm{H}) ;{ }^{13} \mathrm{C} \mathrm{NMR}\left(\mathrm{CDCl}_{3}\right) \delta 221.5,145.6,139.6,138.1$, $130.5,127.0,120.9,92.6,80.4,59.4,50.3,48.0,44.2,37.8,35.9,31.5,29.2,26.2$, 25.7, 21.6, 13.8; LRMS (EI): $m / z$ (relative intensity), $512(\mathrm{M}+2,89), 510\left(\mathrm{M}^{+}, 85\right), 495(22), 493(22), 468$ (23), 466 (23), 455 (22), 453 (23), 363 (55), 299 (95), 282 (100), 226 (55), 159 (57), 97 (71); HRMS (EI) calcd. for $\mathrm{C}_{21} \mathrm{H}_{25} \mathrm{Cl}_{3} \mathrm{O}_{6} \mathrm{~S} 510.0437$, found 510.0440 .

2-Fluoromethylestra-1,3,5(10)-triene-17-one-3-(2,2,2-trichloroethyl) sulfate (47). This was prepared from 46 using the procedure described for 45 except 2.6 equiv. of DAST was used and reaction time was shorter ( $3 \mathrm{~h}$ at room temperature). Column chromatography (silica, 9:4 
hexane/ethyl acetate) yielded 47 as white foam in 90\% yield. ${ }^{1} \mathrm{H}$ NMR $\left(\mathrm{CDCl}_{3}\right) \delta 7.39(\mathrm{~s}, 1 \mathrm{H})$, $7.18(\mathrm{~s}, 1 \mathrm{H}), 5.43(\mathrm{~d}, 2 \mathrm{H}, J=48 \mathrm{~Hz}), 4.98(\mathrm{~s}, 2 \mathrm{H}), 2.80-3.0(\mathrm{~m}, 2 \mathrm{H}), 1.83-2.60(\mathrm{~m}, 7 \mathrm{H}), 1.37-$ $1.70(\mathrm{~m}, 7 \mathrm{H}), 0.88(\mathrm{~s}, 3 \mathrm{H}) ;{ }^{13} \mathrm{C} \mathrm{NMR}\left(\mathrm{CDCl}_{3}\right) \delta 220.5,146.0(\mathrm{~d}, J=6 \mathrm{~Hz}), 140.1(\mathrm{~s}, 1 \mathrm{H}), 140.1$ $(\mathrm{d}, J=1 \mathrm{~Hz}), 127.8(\mathrm{~d}, J=6 \mathrm{~Hz}), 125.6(\mathrm{~d}, J=14 \mathrm{~Hz}), 121.2(\mathrm{~d}, J=2 \mathrm{~Hz}), 92.5,80.5(\mathrm{~d}, J=2$ $\mathrm{Hz}), 79.6(\mathrm{~d}, J=167 \mathrm{~Hz}), 50.4,47.9,44.1,37.8,35.9,31.5,29.4,26.1,25.8,21.6,13.9 ;{ }^{19} \mathrm{~F}$ NMR (proton coupled, $\left.\mathrm{CDCl}_{3}\right) \delta-132(\mathrm{t}, J=47 \mathrm{~Hz}$ ); LRMS (EI): $\mathrm{m} / z$ (relative intensity), 514 (M+2, 100), $512\left(\mathrm{M}^{+}, 98\right), 470$ (42), 468 (37), 457 (35), 455 (38), 395 (30), 283 (58), 245 (93), 97 (85); HRMS (EI) calcd. for $\mathrm{C}_{21} \mathrm{H}_{24} \mathrm{Cl}_{3} \mathrm{FO}_{5} \mathrm{~S} 512.0394$, found 512.0396.

Ammonium 2-fluoromethyl-3-(sulfonatooxy)estra-1,3,5(10)-triene-17-one (2). To a solution of the ester 49 (395 mg, $0.77 \mathrm{mmol})$ in HPLC grade $\mathrm{MeOH}(20 \mathrm{~mL})$ was added ammonium formate $(291 \mathrm{mg}, 4.62 \mathrm{mmol})$ and $80 \mathrm{mg}(20 \mathrm{wt} . \%)$ of $10 \% \mathrm{Pd} / \mathrm{C}$. The reaction mixture was stirred for $24 \mathrm{~h}$. After filtration and removal of $\mathrm{MeOH}$, the residue was charged with ammonium formate $(260 \mathrm{mg}, 4.13 \mathrm{mmol}), 20 \mathrm{mg}$ of Pd black and $\mathrm{MeOH}(8 \mathrm{~mL})$. The reaction mixture was stirred for $24 \mathrm{~h}$. The Pd catalyst was filtered away and the filtrate was concentrated in vacuo to give a white residue. The residue was dissolved in water and lyophilized to give a white fluffy solid. This solid was dissolved in a minimal amount of $\mathrm{CH}_{2} \mathrm{Cl}_{2} / \mathrm{MeOH} / \mathrm{NH}_{4} \mathrm{OH}$ (5:4:0.5) and purified by flash chromatography using $\mathrm{CH}_{2} \mathrm{Cl}_{2} / \mathrm{MeOH} / \mathrm{NH}_{4} \mathrm{OH}$ (10:2:0.5) as eluant. Fractions containing 2 were pooled, concentrated and the residue dissolved in water and lyophilized. The lyophilization procedure was repeated. This gave compound $\mathbf{2}$ as a white fluffy ammonium salt in $75 \%$ yield. ${ }^{1} \mathrm{H}$ NMR $\left(\mathrm{CD}_{3} \mathrm{OD}\right) \delta 7.34(\mathrm{~s}, 1 \mathrm{H}), 7.16(\mathrm{~s}, 1 \mathrm{H}), 5.46(\mathrm{~d}, 2 \mathrm{H}, J=48 \mathrm{~Hz}), 4.87(\mathrm{~s}$, $4 \mathrm{H}), 2.81-3.07(\mathrm{~m}, 2 \mathrm{H}), 1.80-2.58(\mathrm{~m}, 7 \mathrm{H}), 1.37-1.71(\mathrm{~m}, 6 \mathrm{H}), 0.90(\mathrm{~s}, 3 \mathrm{H}) ;{ }^{13} \mathrm{C} \mathrm{NMR}\left(\mathrm{CD}_{3} \mathrm{OD}\right)$ $\delta 222.5,148.0(\mathrm{~d}, J=5 \mathrm{~Hz}), 138.4(\mathrm{~d}, J=3 \mathrm{~Hz}), 136.9(\mathrm{~d}, J=2 \mathrm{~Hz}), 126.6(\mathrm{~d}, J=17 \mathrm{~Hz}), 126.0$ (d, $J=7 \mathrm{~Hz}), 121.9(\mathrm{~d}, J=1 \mathrm{~Hz}), 80.0(\mathrm{~d}, J=159 \mathrm{~Hz}), 50.2,47.9,44.0,38.1,35.5,31.4,29.0$, 26.1, 25.6, 21.2, 13.0; ${ }^{19} \mathrm{~F}$ NMR (proton coupled, $\left.\mathrm{CD}_{3} \mathrm{OD}\right) \delta-134(\mathrm{t}, J=52 \mathrm{~Hz}$ ); LRMS (ESI) $m / z$ (relative intensity), $381.1\left(\mathrm{M}-\mathrm{NH}_{4}^{+}, 23\right), 299.2\left(\mathrm{M}-\mathrm{SO}_{3} \mathrm{NH}_{4}^{+}-\mathrm{F}-+\mathrm{OH}, 100\right)$; HRMS (ESI) calcd. for $\mathrm{C}_{19} \mathrm{H}_{22} \mathrm{O}_{5} \mathrm{FS} 381.1177$, found 381.1186; calcd. for $\mathrm{C}_{19} \mathrm{H}_{23} \mathrm{O}_{6} \mathrm{~S} 379.1220$, found 379.1226; calcd. for $\mathrm{C}_{19} \mathrm{H}_{23} \mathrm{O}_{3} 299.1652$, found 299.1649.

\section{References}

1. Hedayatullah, M.; .Leveque, J.C.; Denivelle, L. C. R. Acad. Sc. Paris, Serie C, 1972, 274, 1938.

2. Free acid: Bollard, M. E.; Holmes, E.; Blackledge, C. A.; Lindon, J. C.; Wilson, I. D.; Nicholson, J. K. Xenobiotica, 1996, 26; 255 - 274.

3. Potassium salt: Ragan, M. A. Can. J. Chem. 1978, 56, 2681.

4. Potassium salt: Gemborys, M. W.; Grible, G. W. J. Med. Chem. 1978, 21, 649.

5. Potassium salt: ref. 2.

6. Free acid: ref. 2 .

7. Potassium salt: Goossens, H. D.; Lambrechts, H. J. A.; Cerfontain, H.; Wit, P. de Recl. Trav. Chim. Pays-Bas, 1988, 107, 426.

8. Sodium salt: Okamoto, K.; Mizuta, E. Kamiya, K.; Imada, I. Chem. Pharm. Bull. 1985, 33, 3756.

9. Potassium salt: Wit, P. de; Cerfontain, H. Recl. Trav. Chim. Pays-Bas, 1988, 107, 121124. 
10. A substrate used for assaying steroid sulfatase. See: Anderson, C. J.; Lucas, L. J. H.; Widlanski, T. S. J. Amer. Chem. Soc. 1995, 117, 3889. To our knowledge, no NMR data has been reported for any salts of this compound.

11. Na salt: Dusza J P; Joseph, J P; Bernstein, S. Steroids, 1968, 12, 49.

12. Potassium salt of the sulfate moiety and the free carboxylic acid: Nicholson, J. K.; Holmes, E.; Sidelmann, U.; Lindon, J. C.; Wilson, I. D. Pharm. Sci. 1996, 2, 127.

13. Pert, D. J.; Ridley, D. D. Aust. J. Chem. 1989, 42; 405. 
Spectra

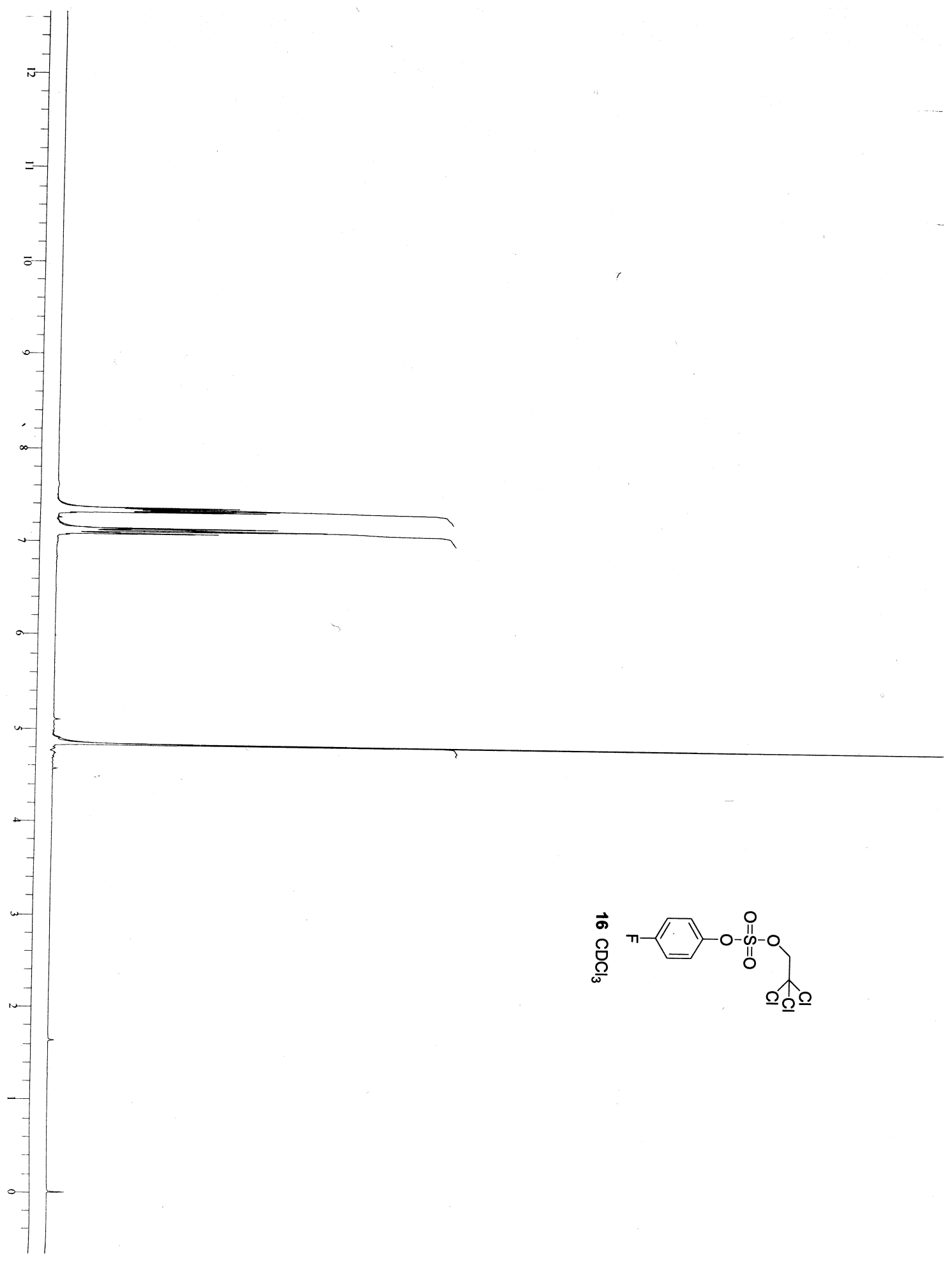




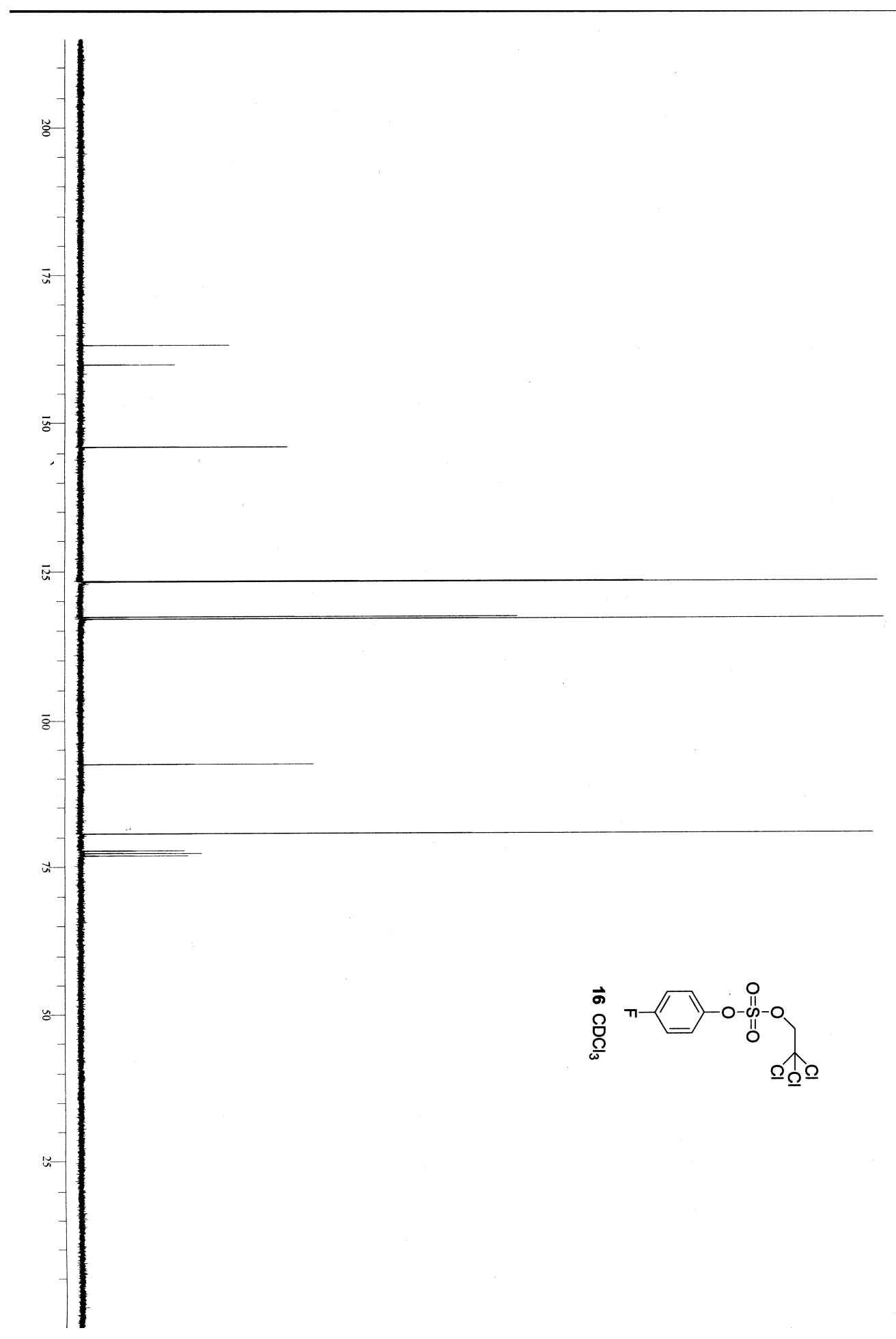




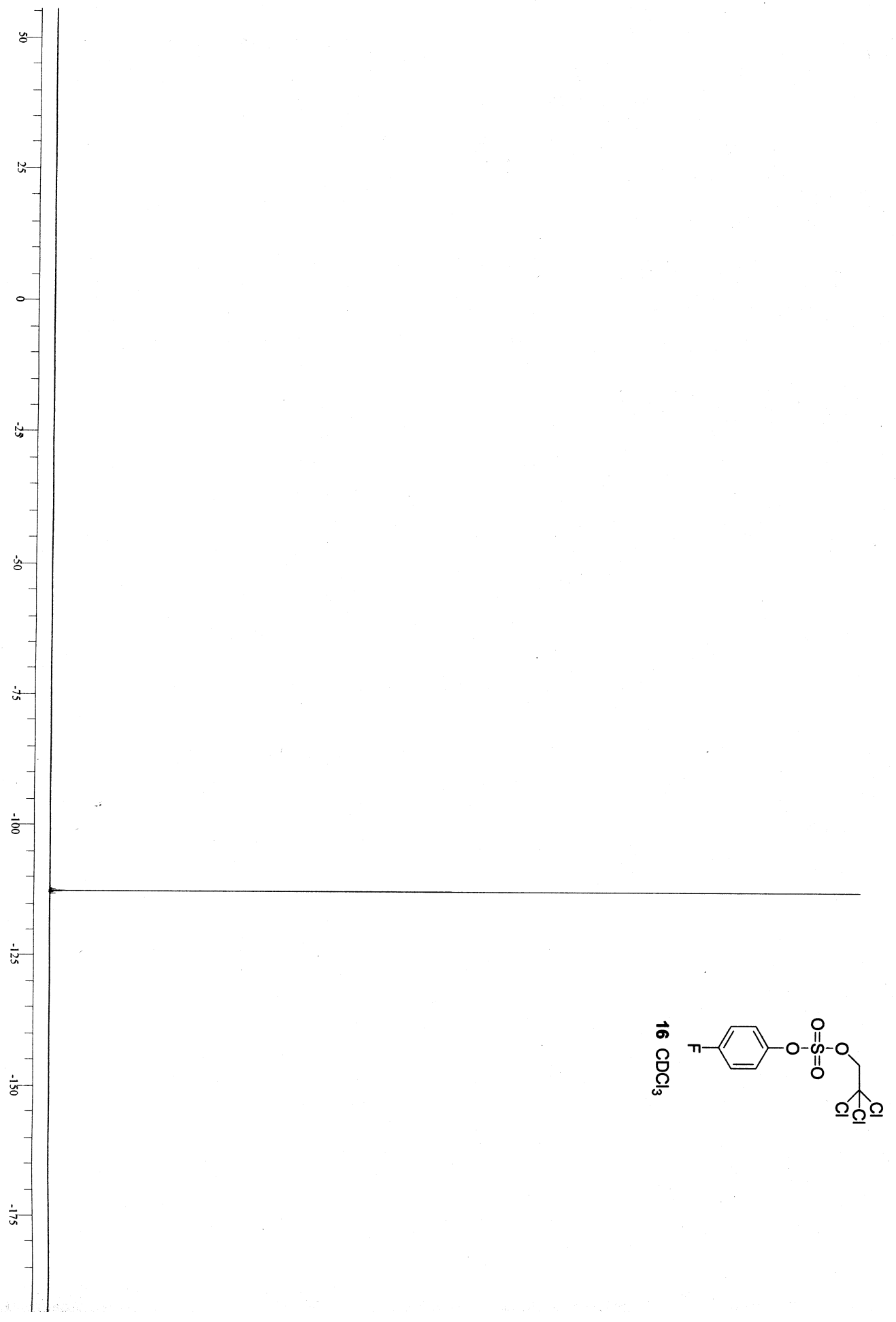




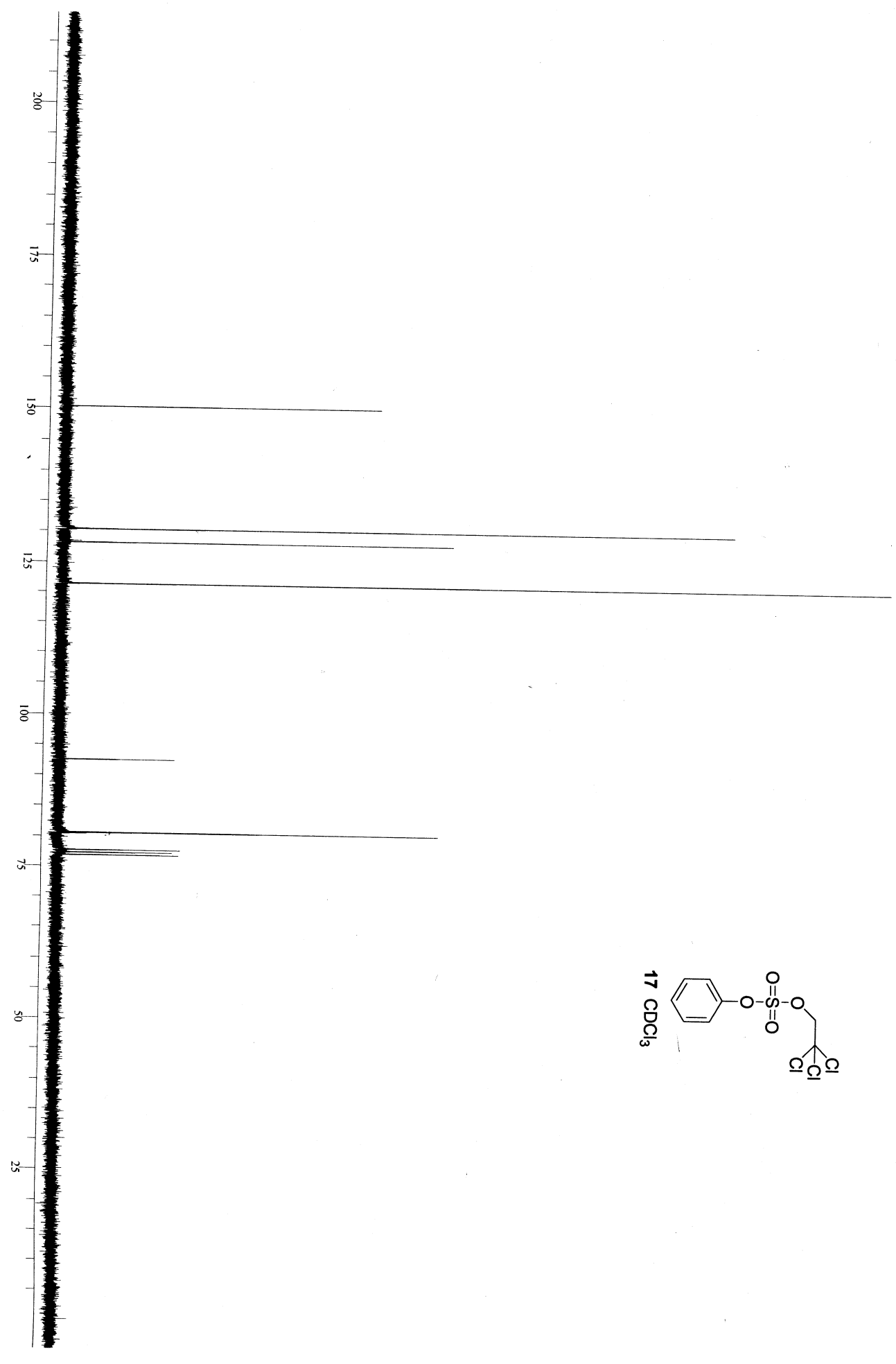




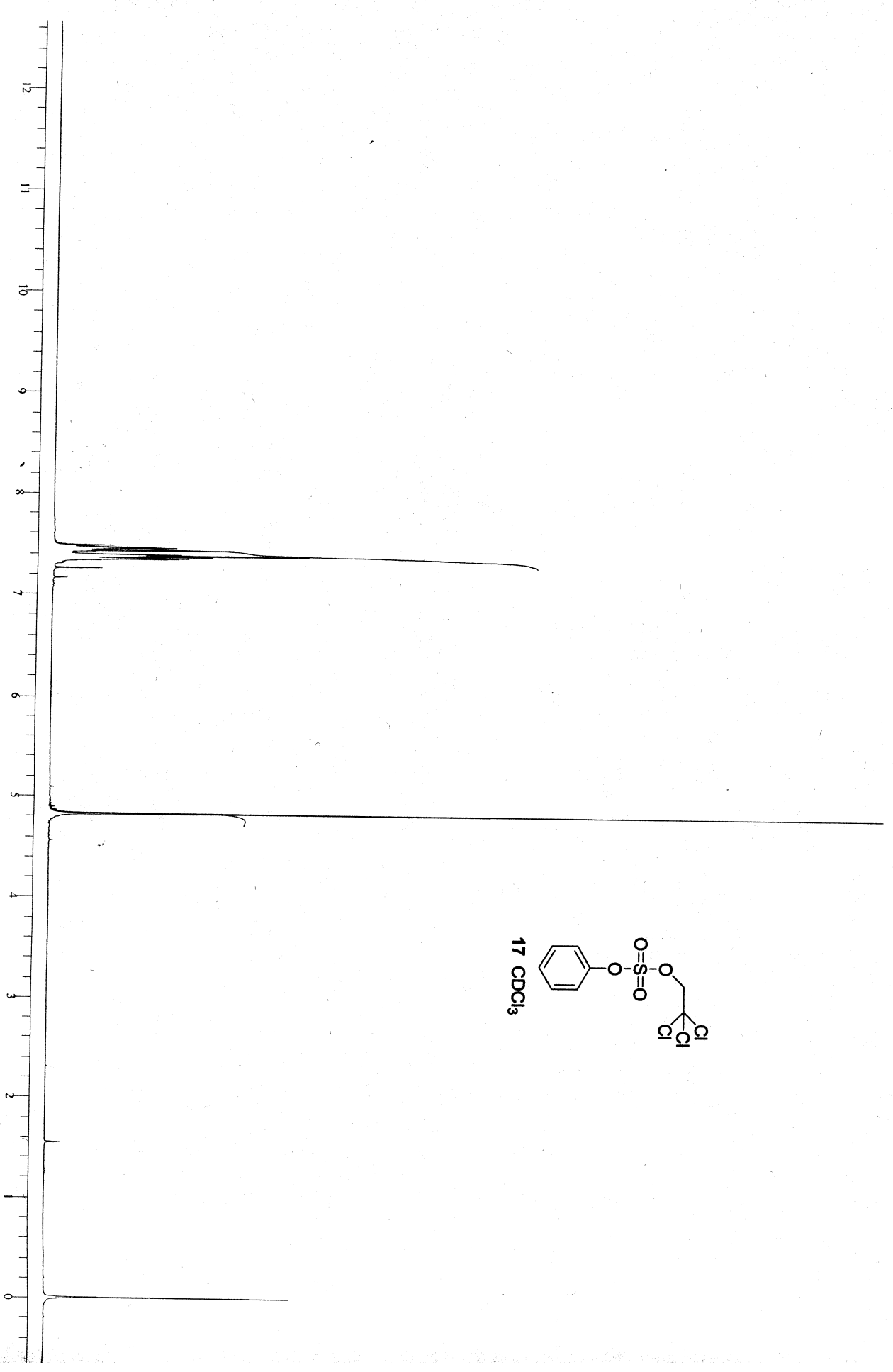




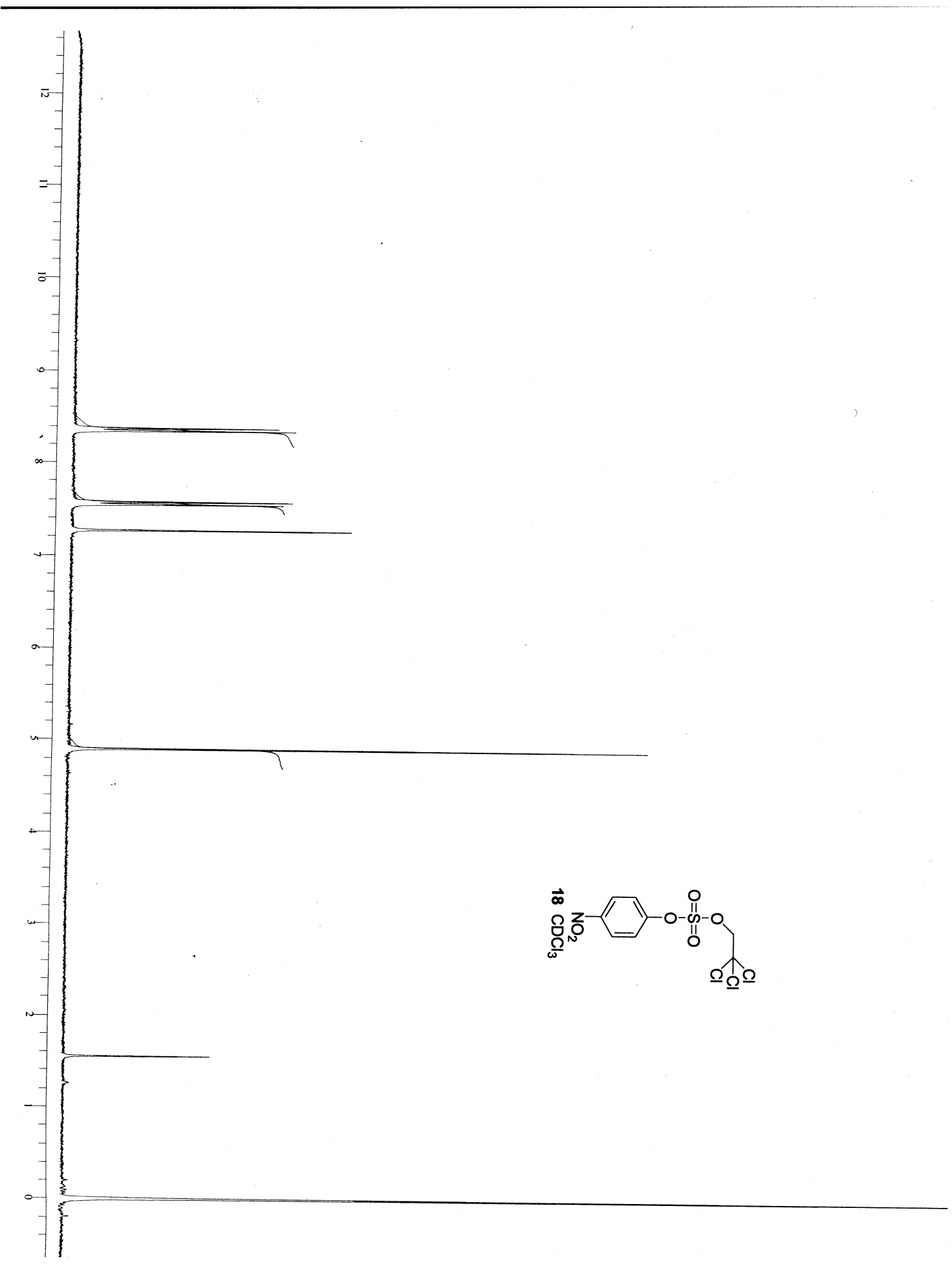




$$
F
$$




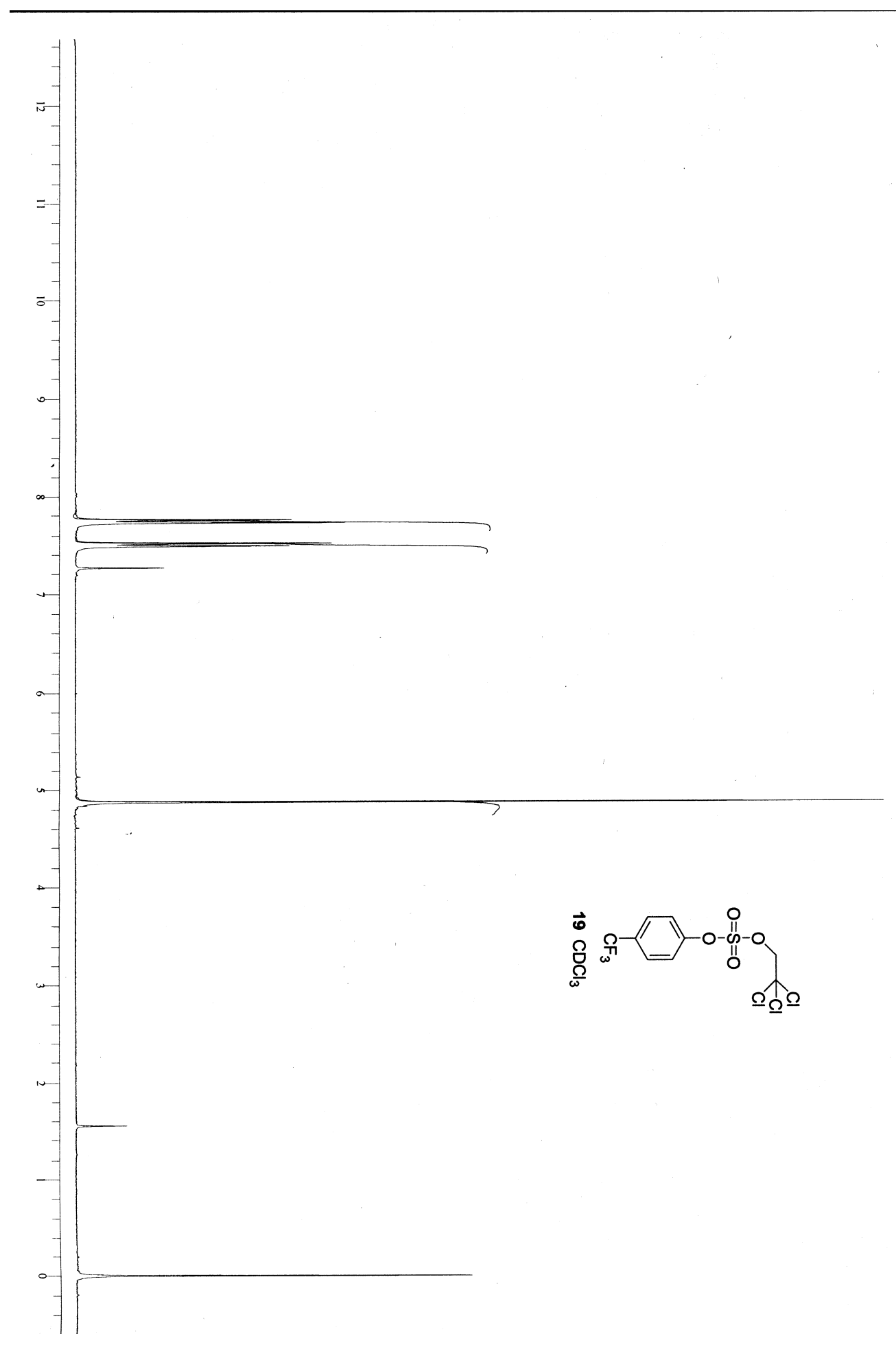




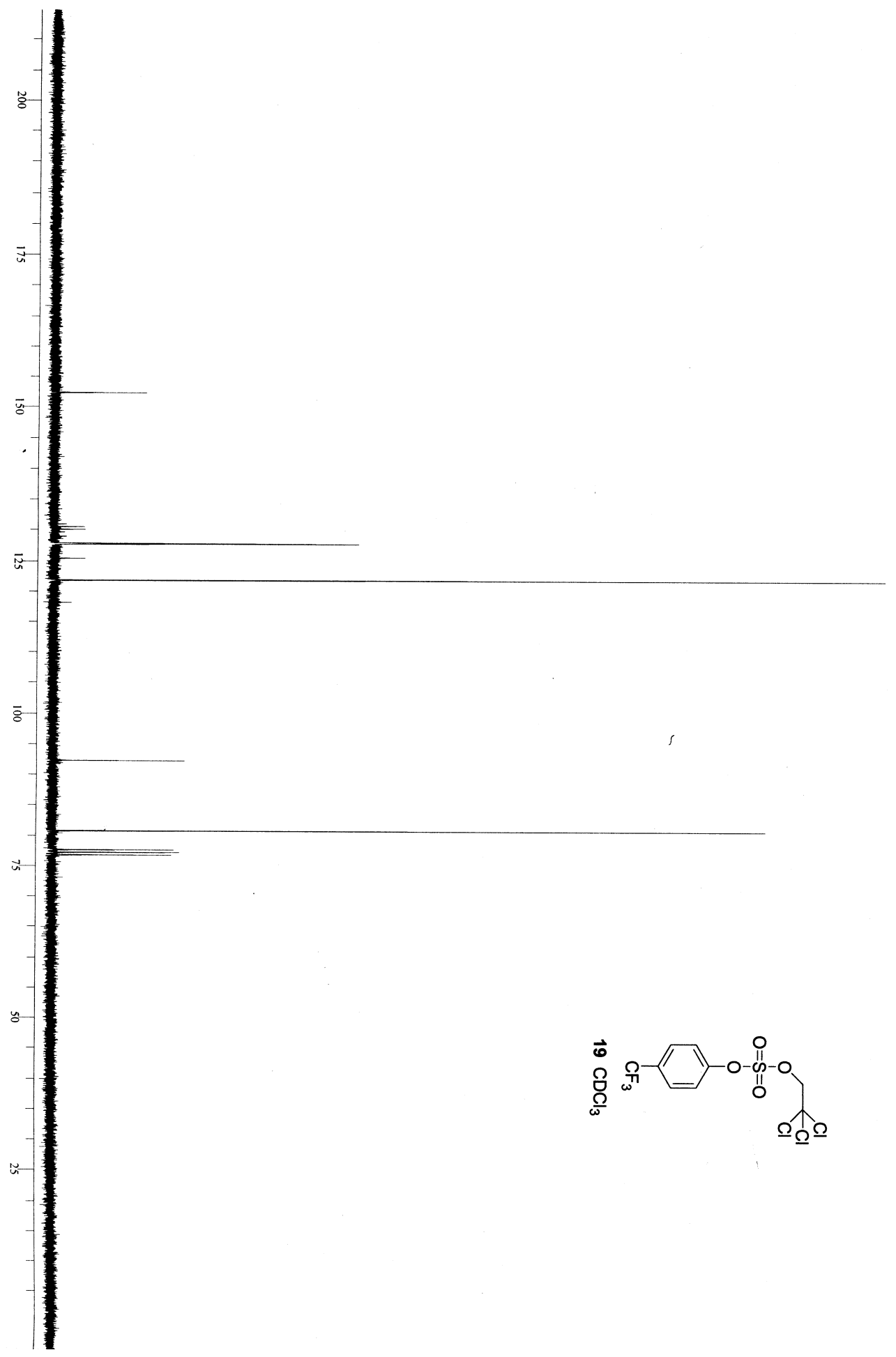




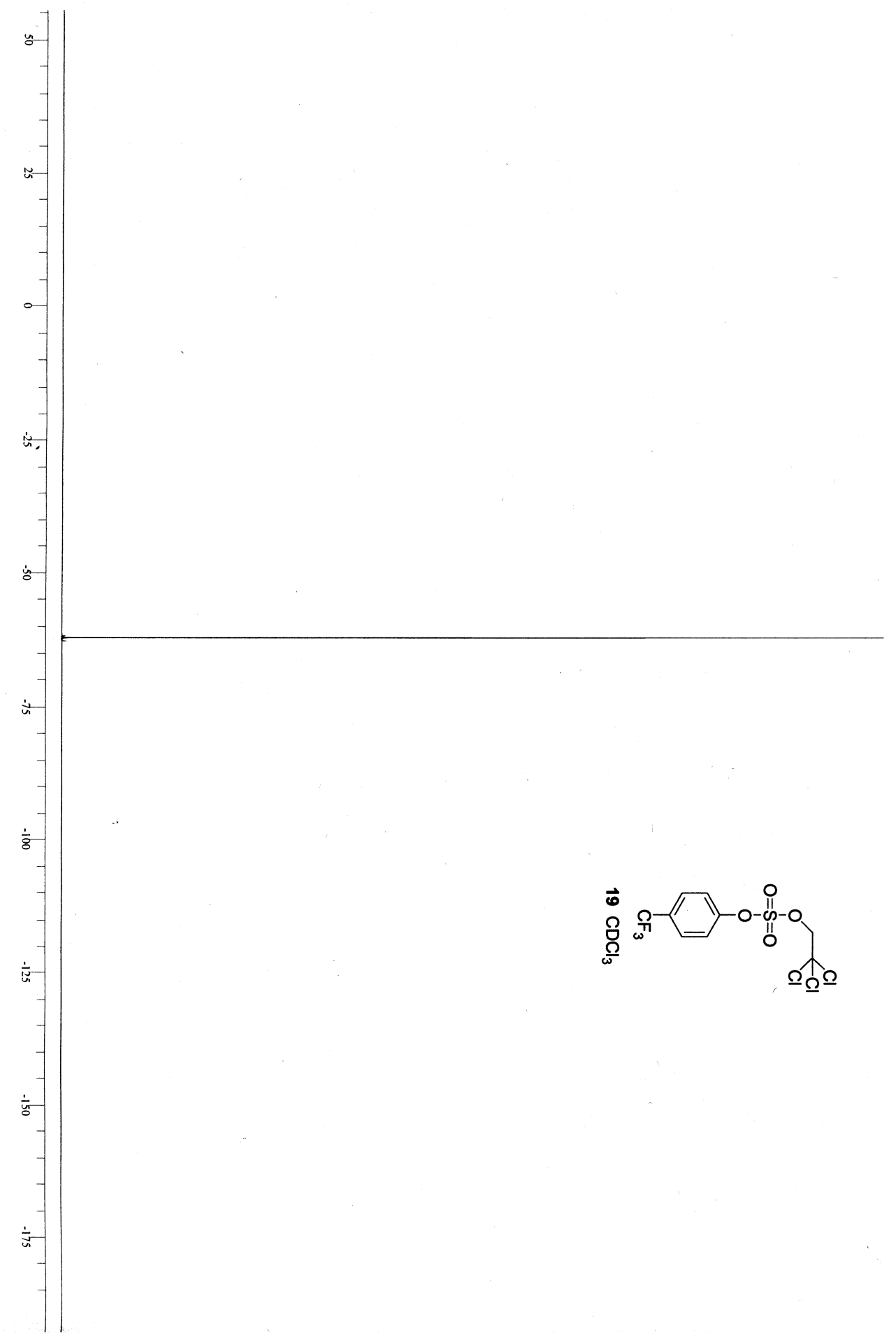




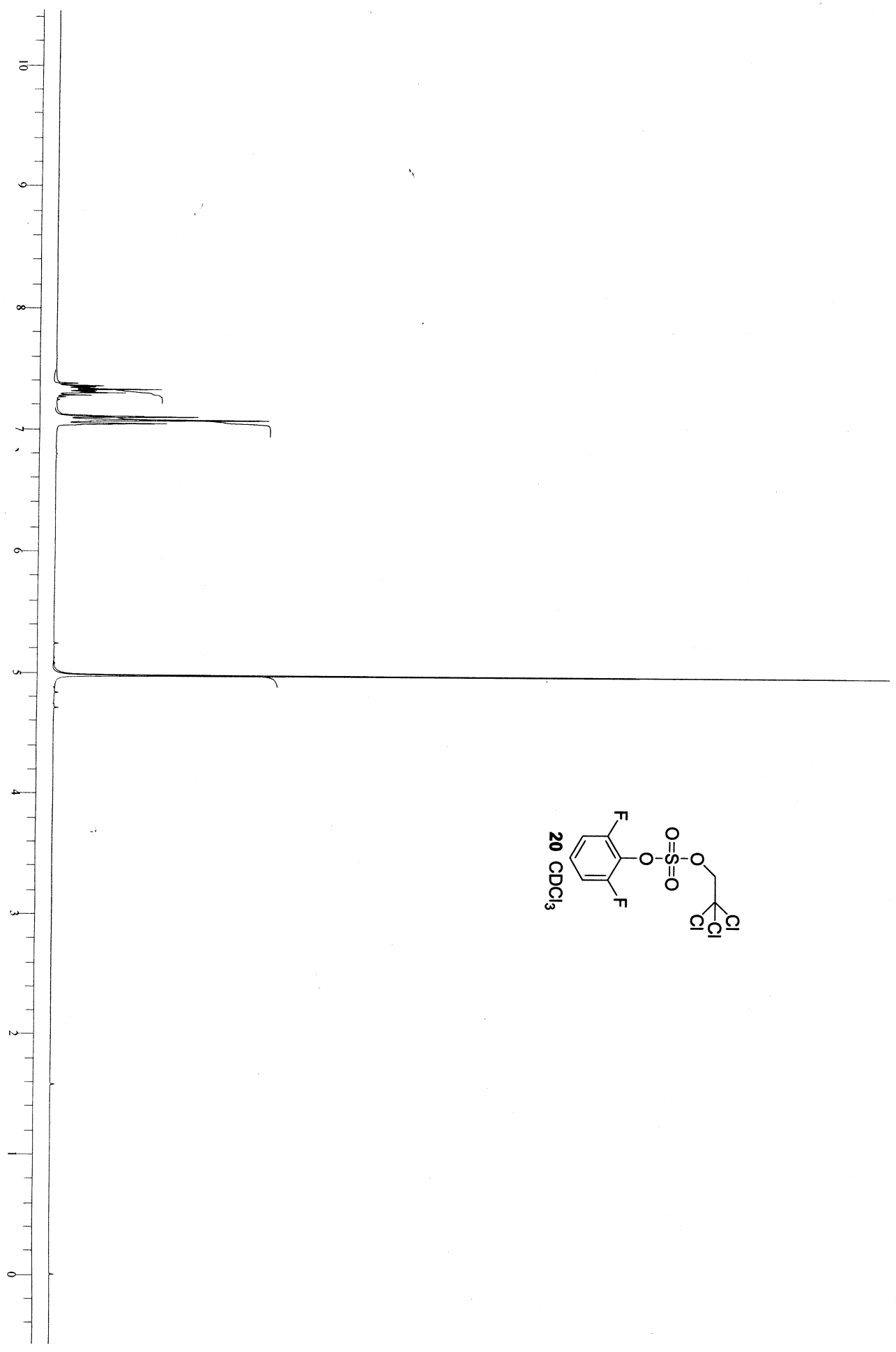




$$
F^{\prime \prime}
$$




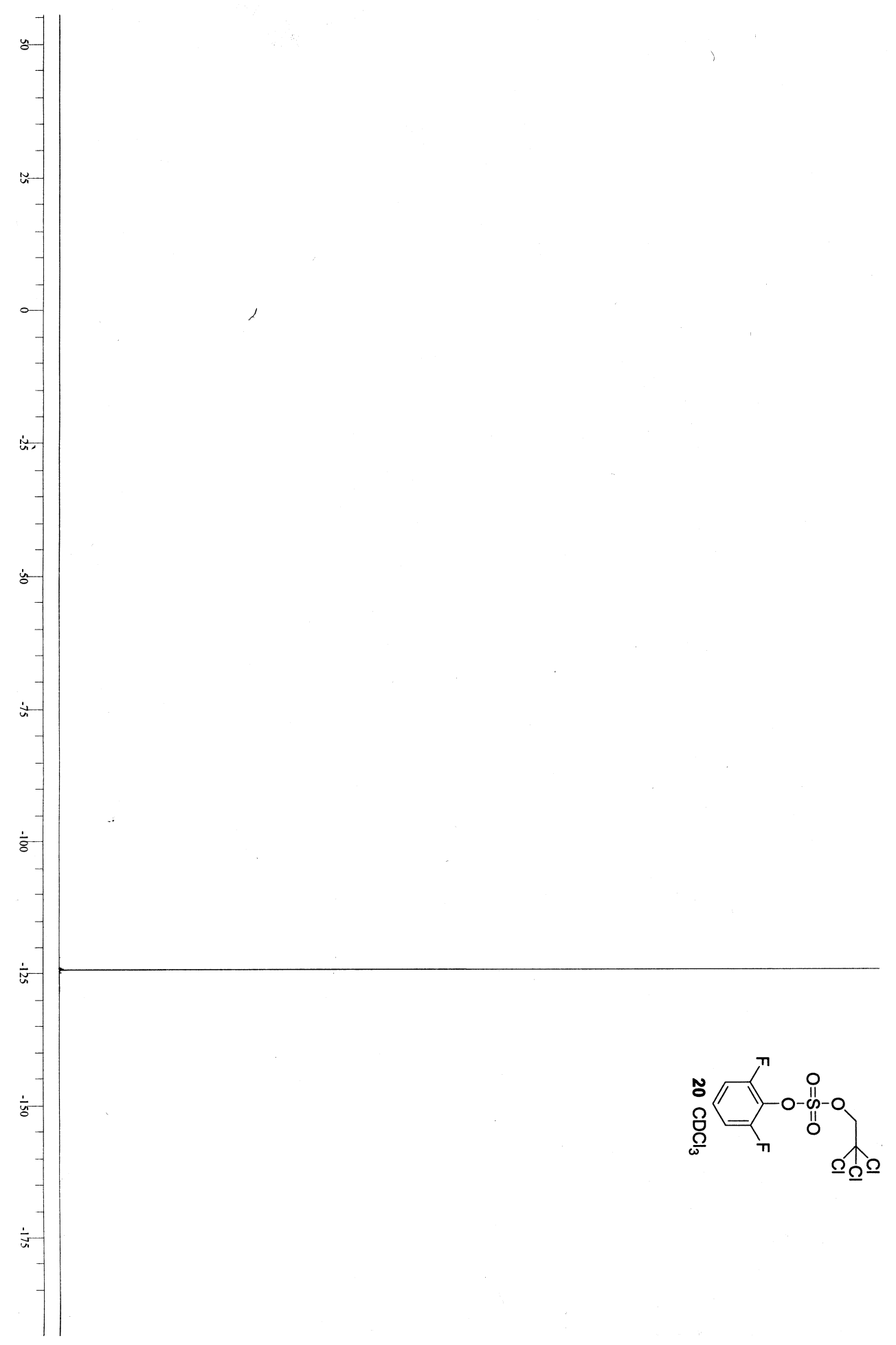




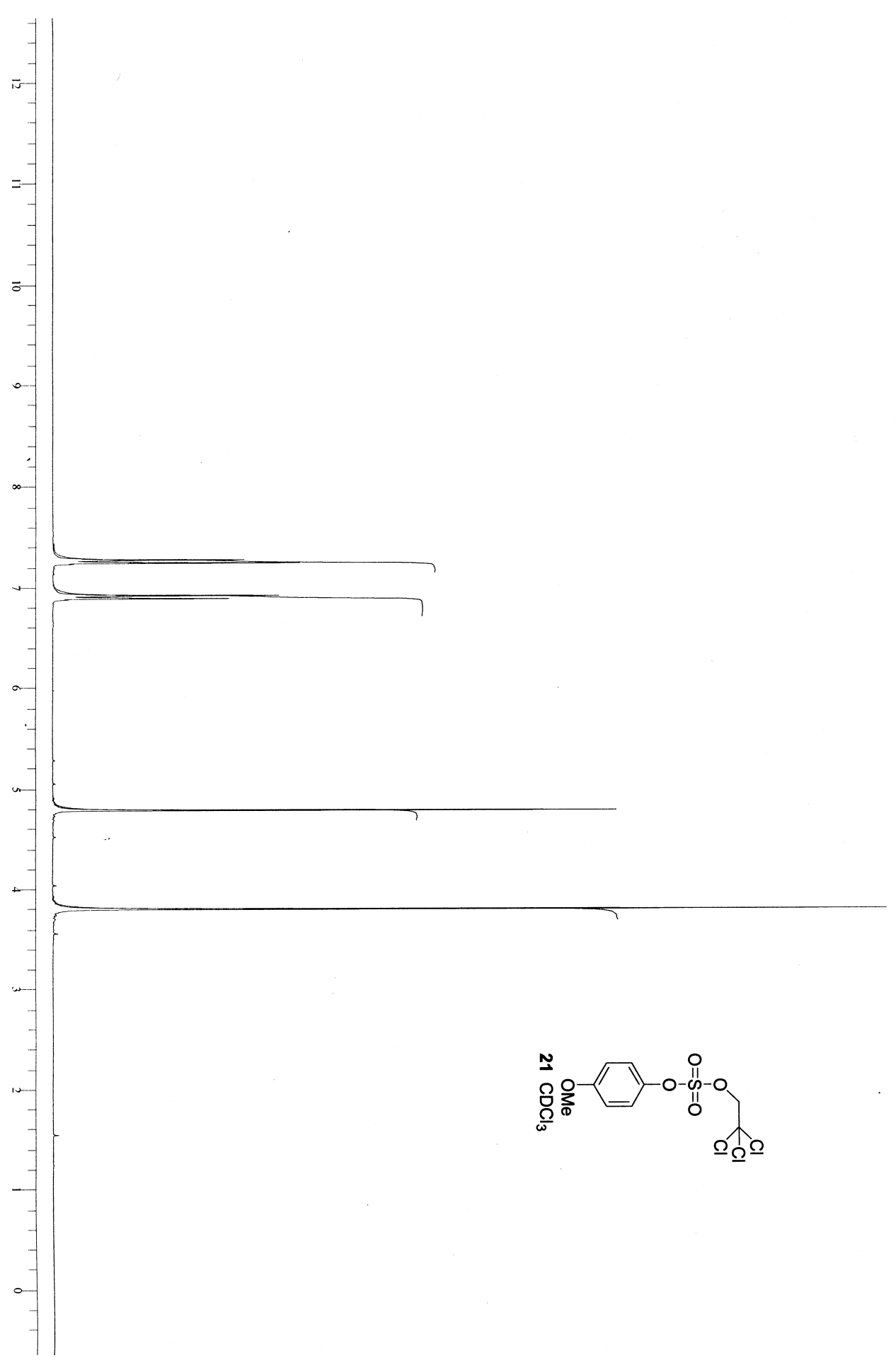




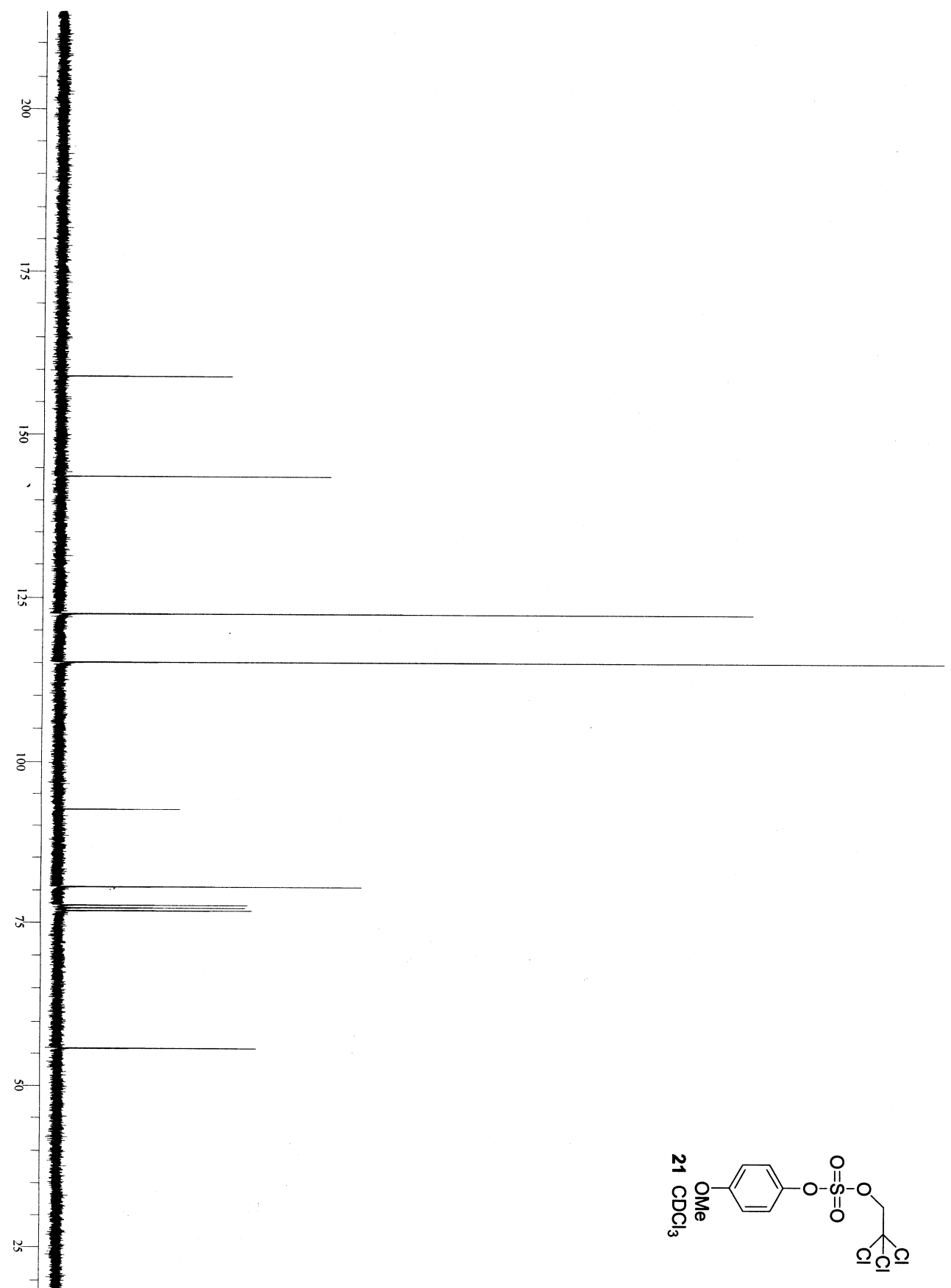

24 


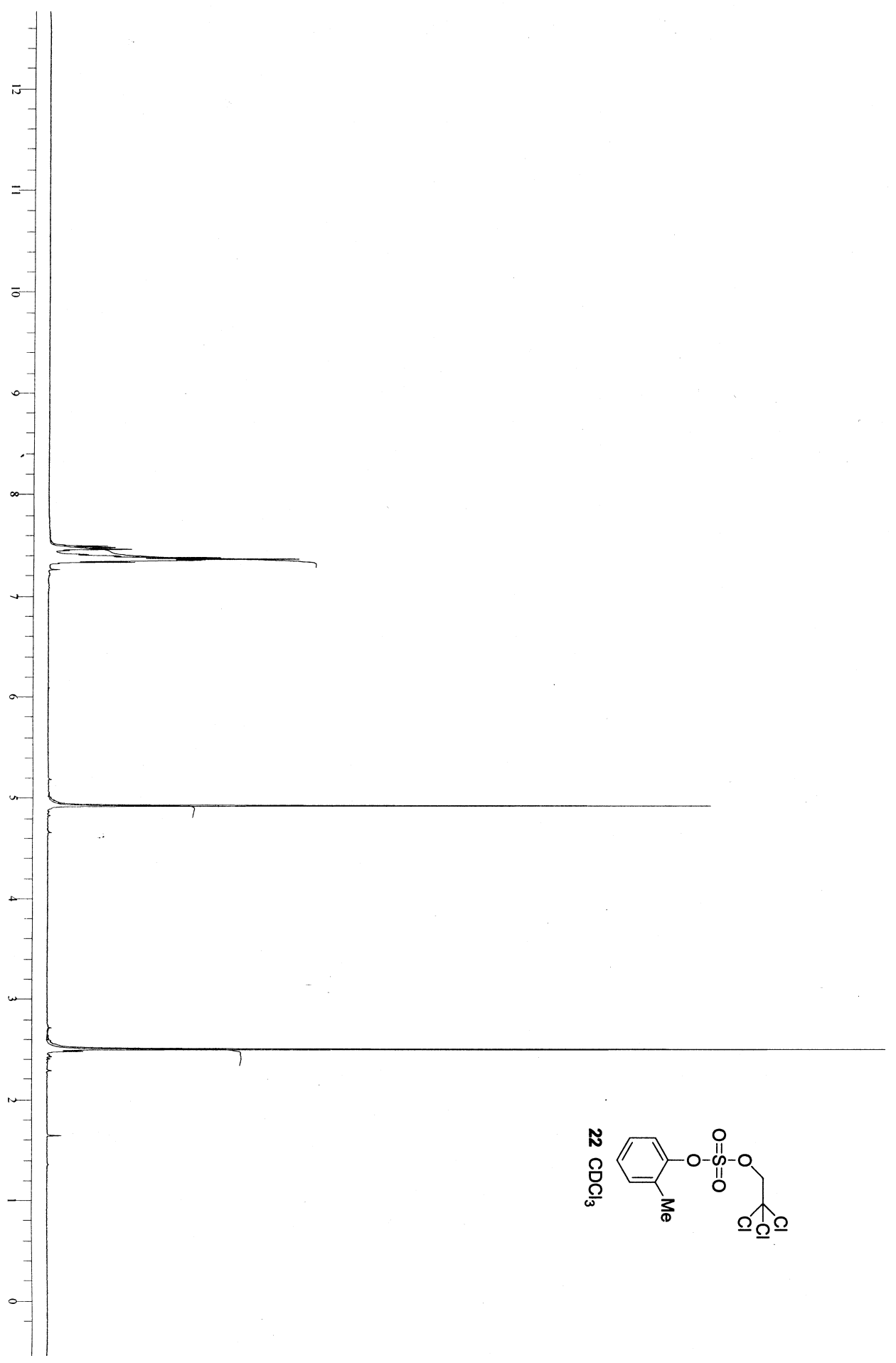




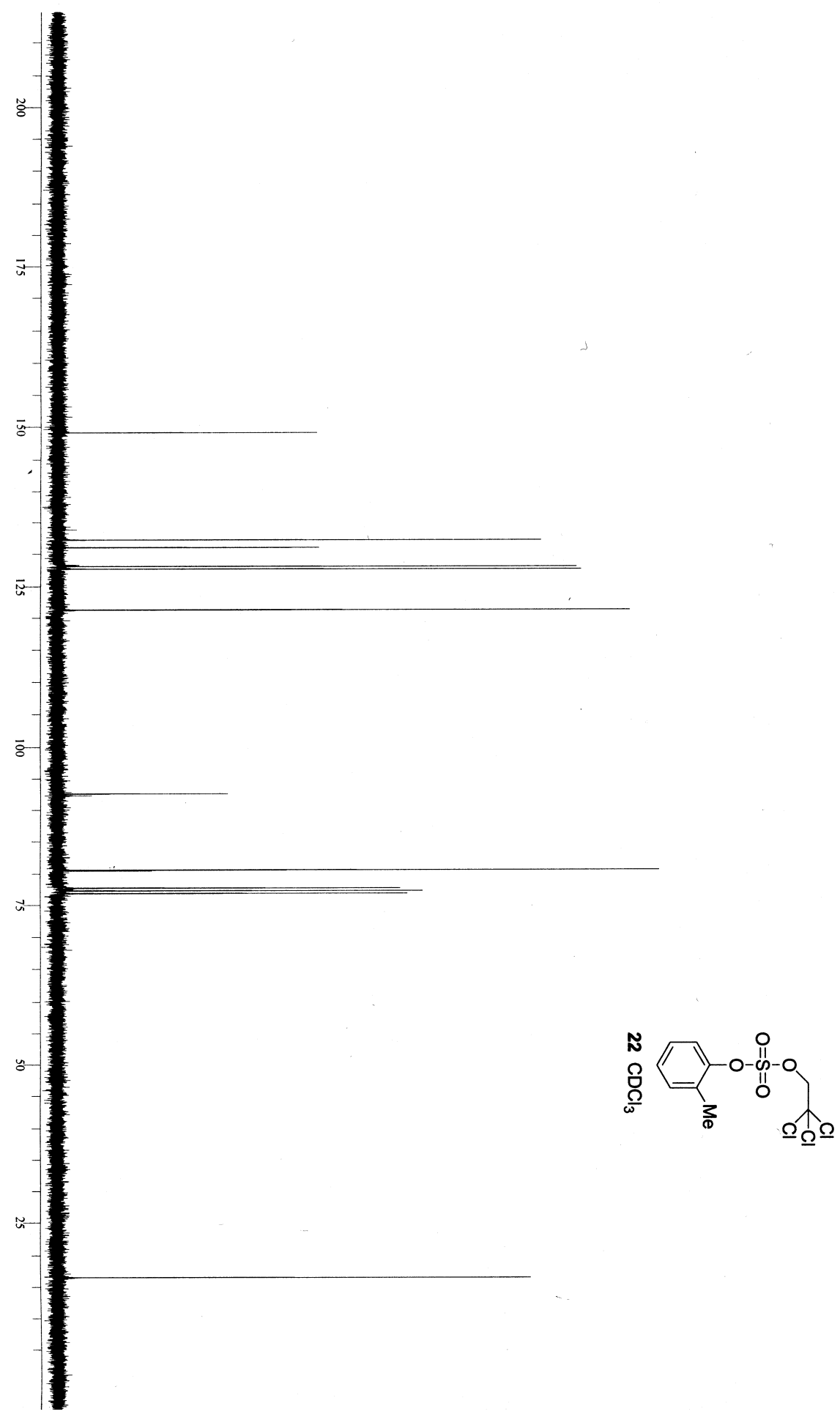

26 


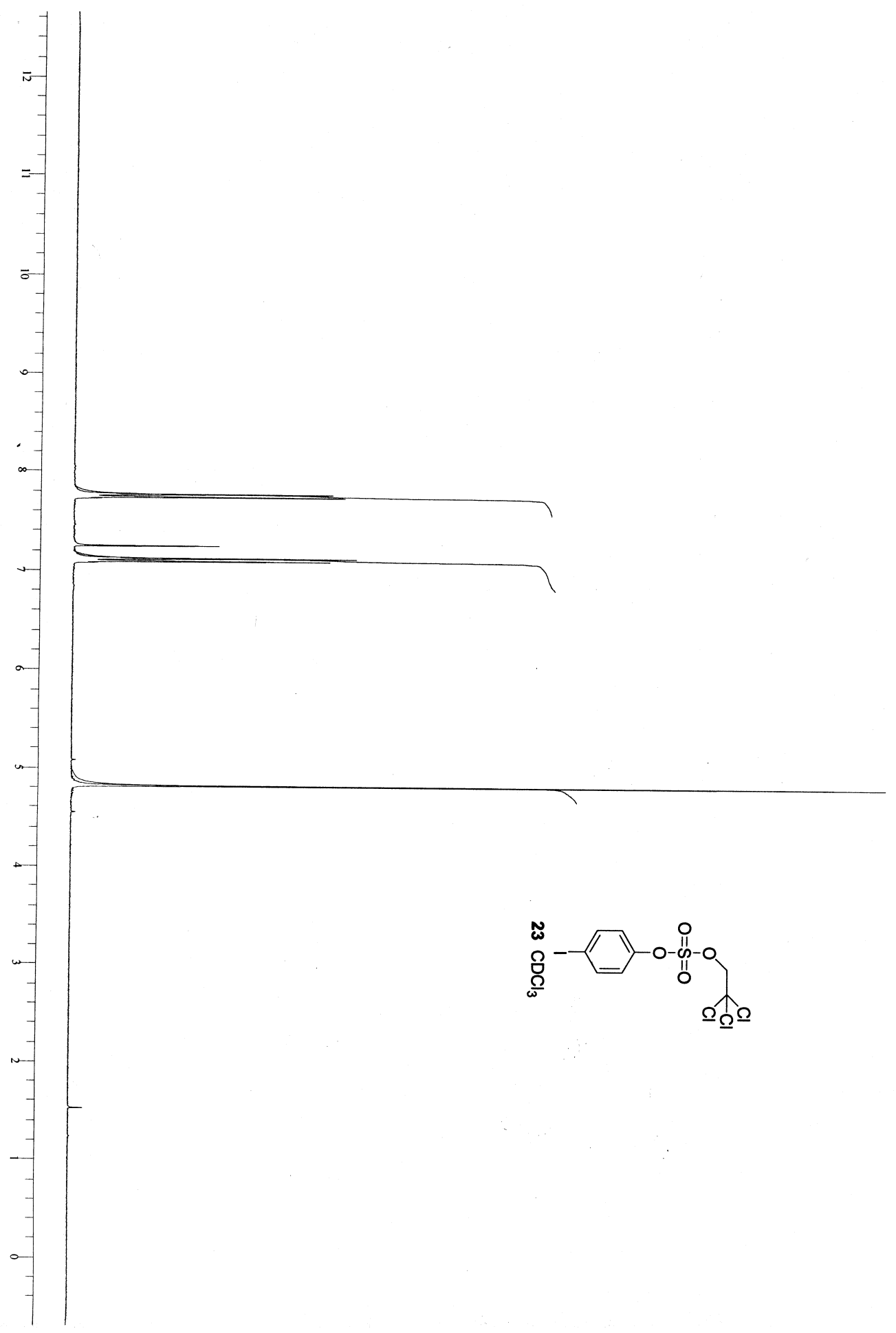




$$
t
$$




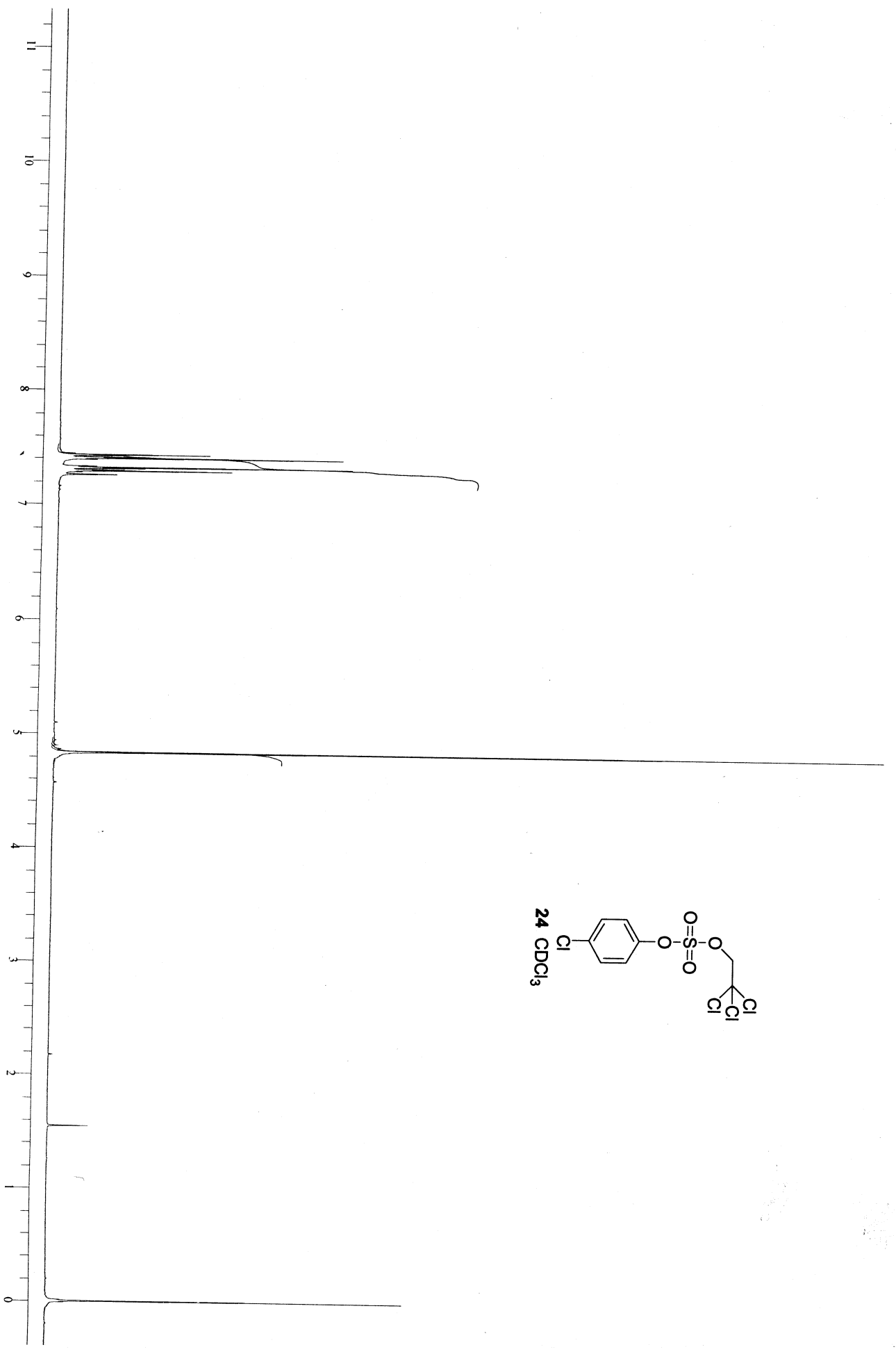




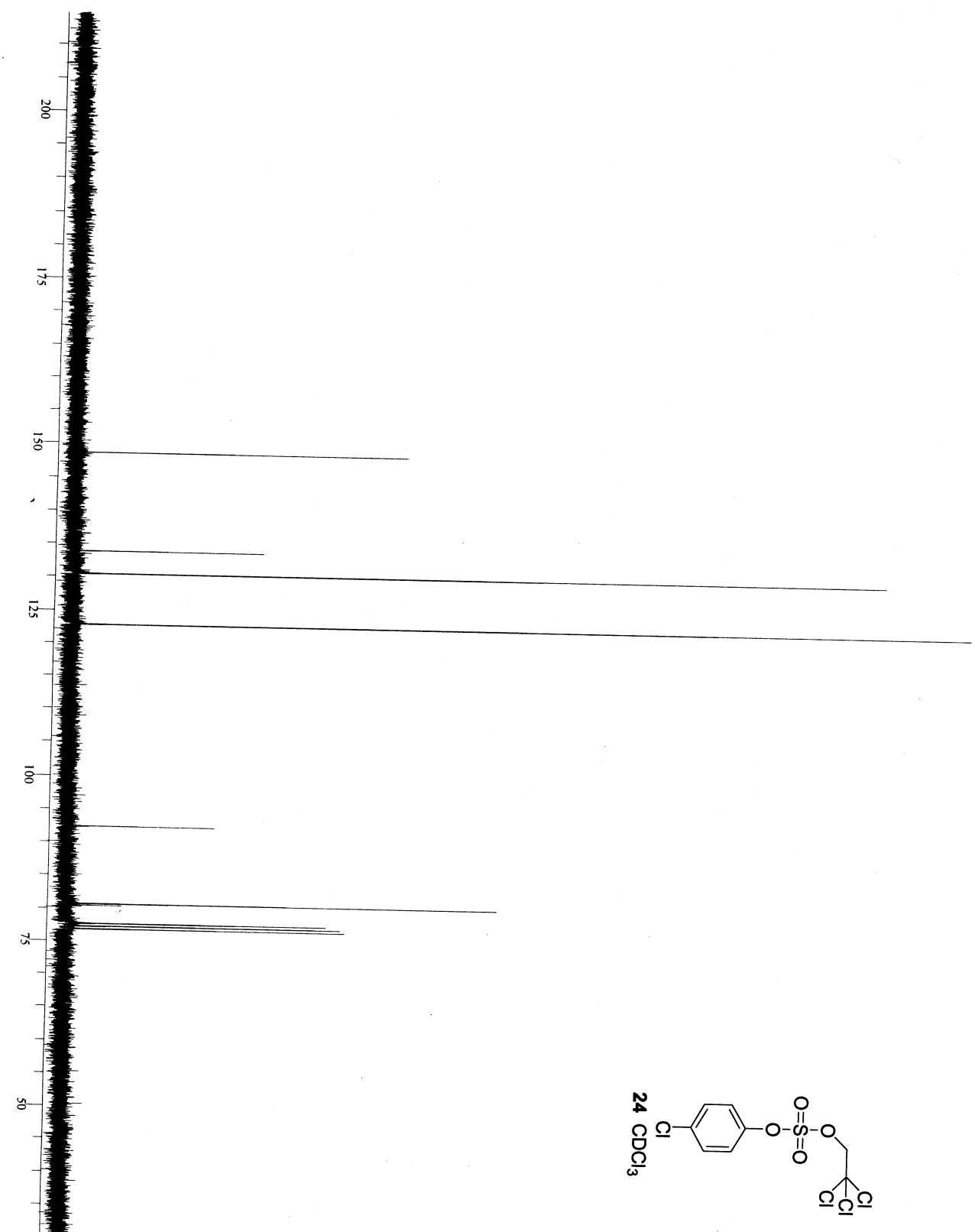

30 


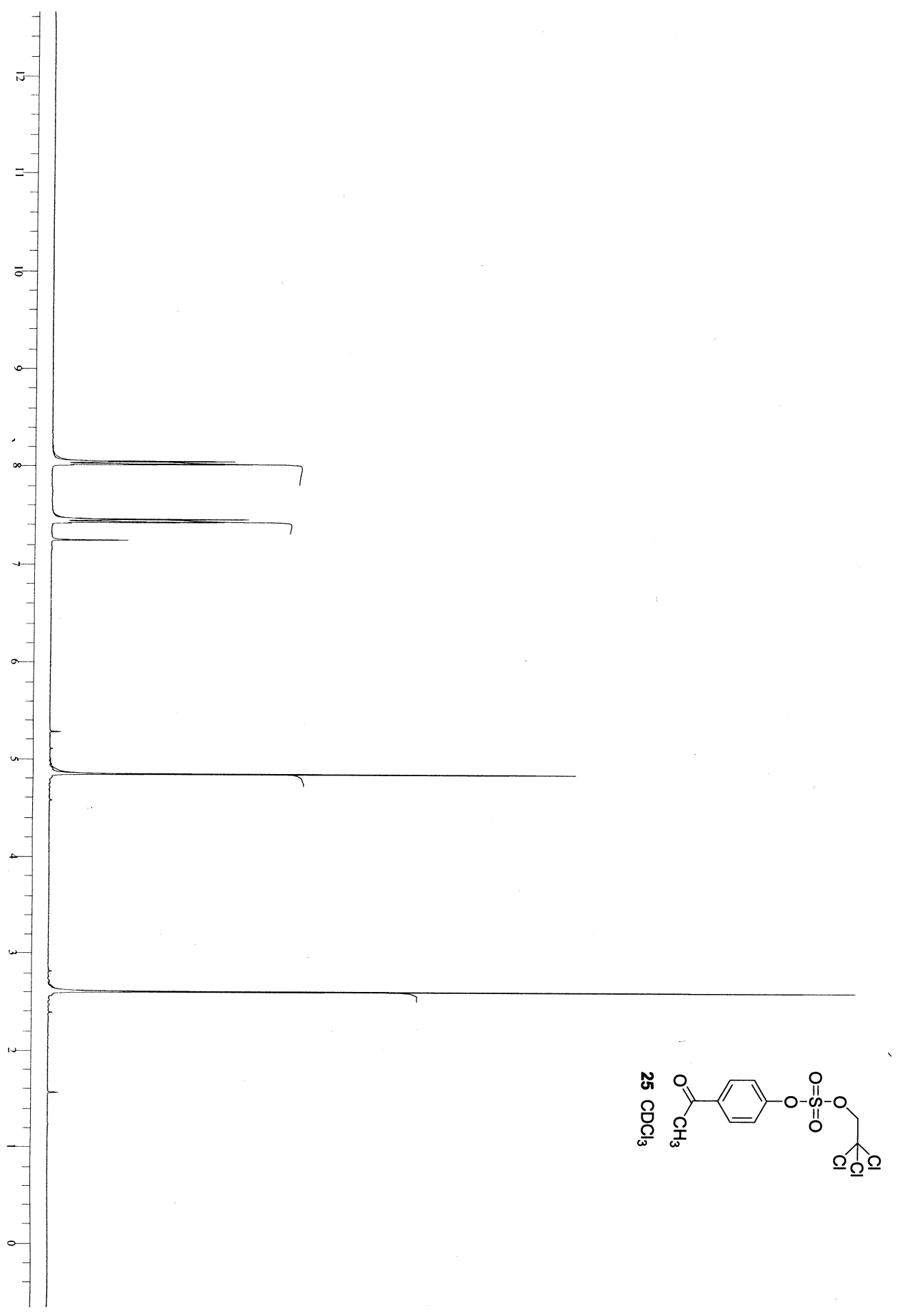




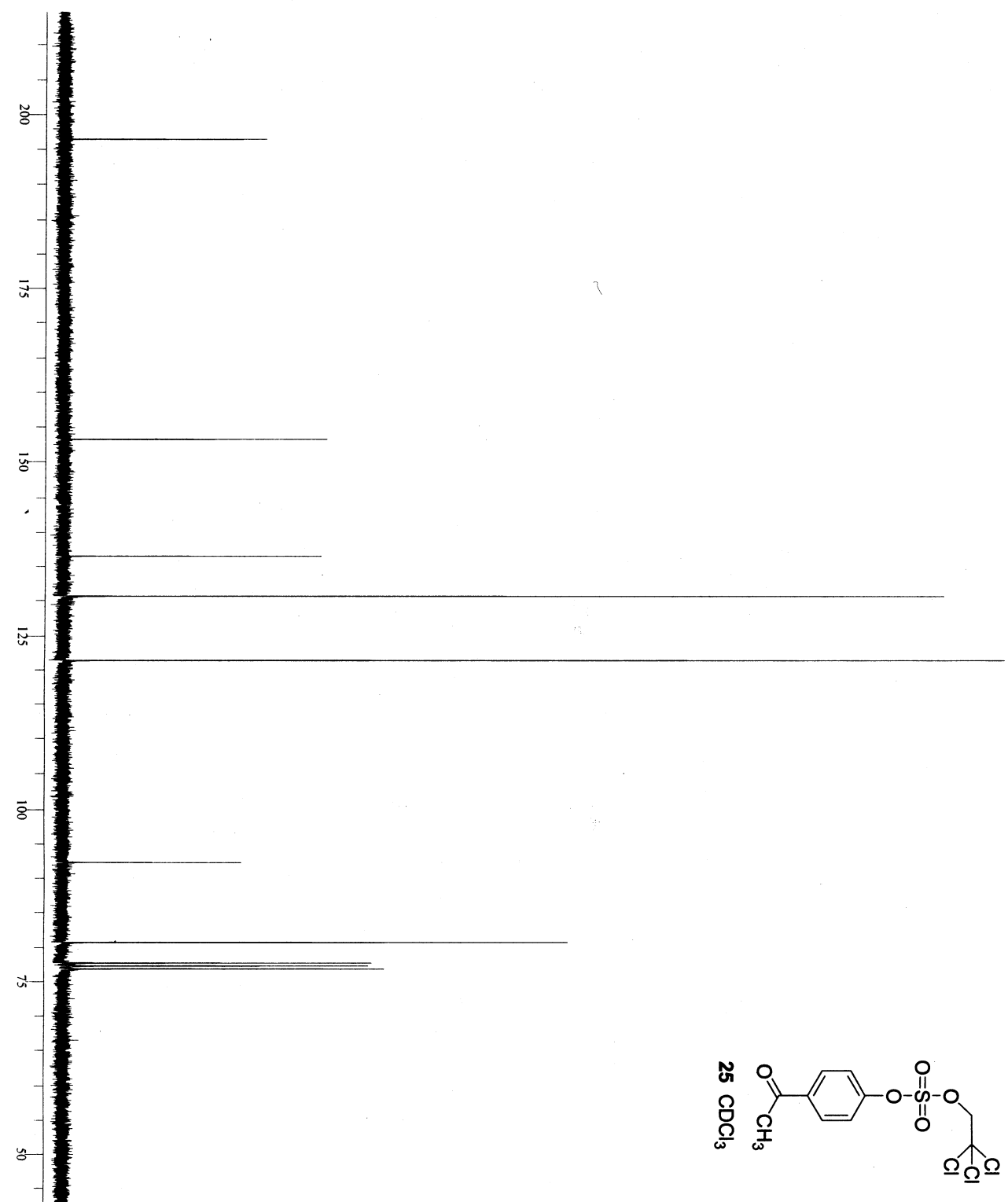

32 


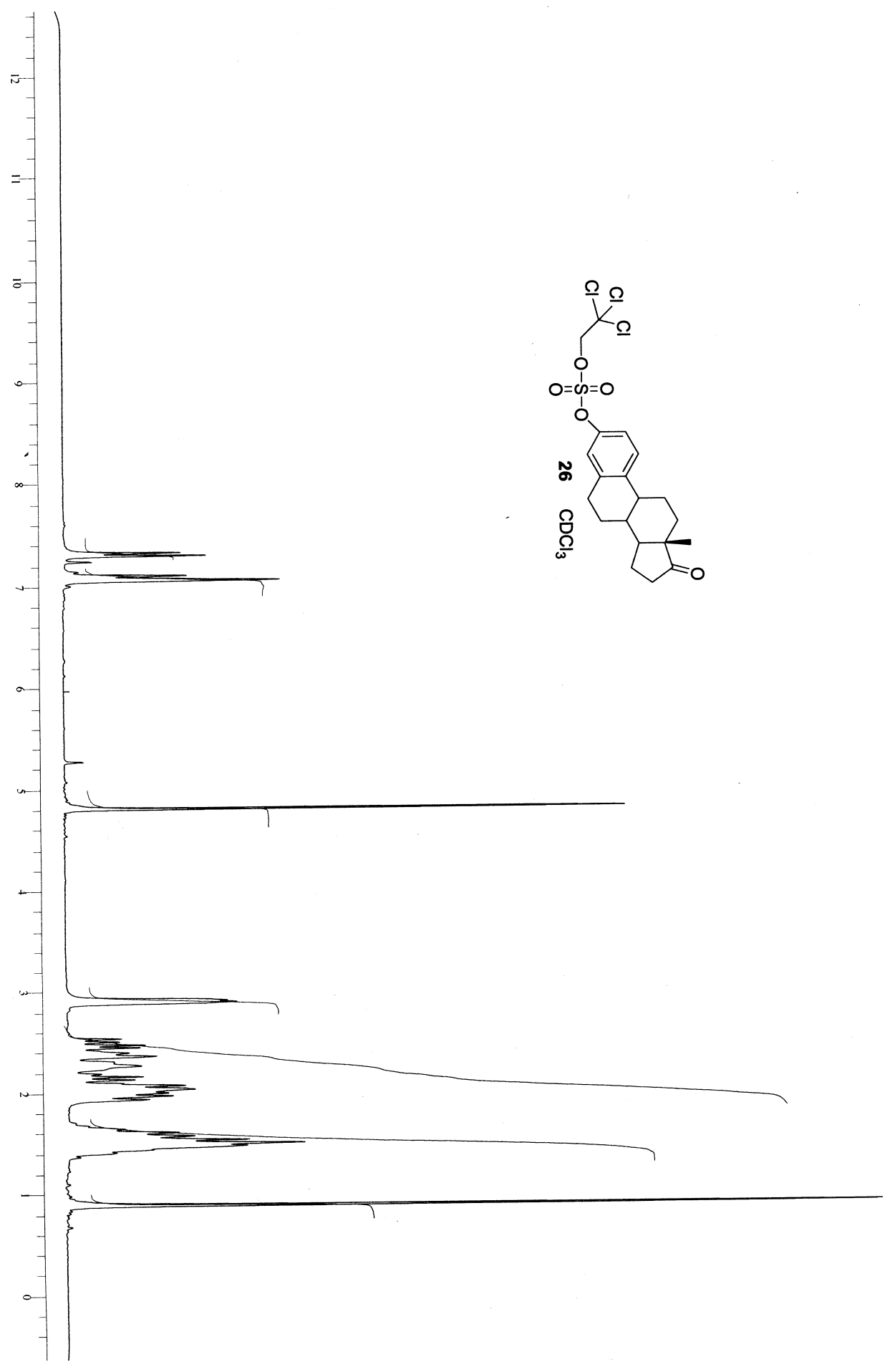




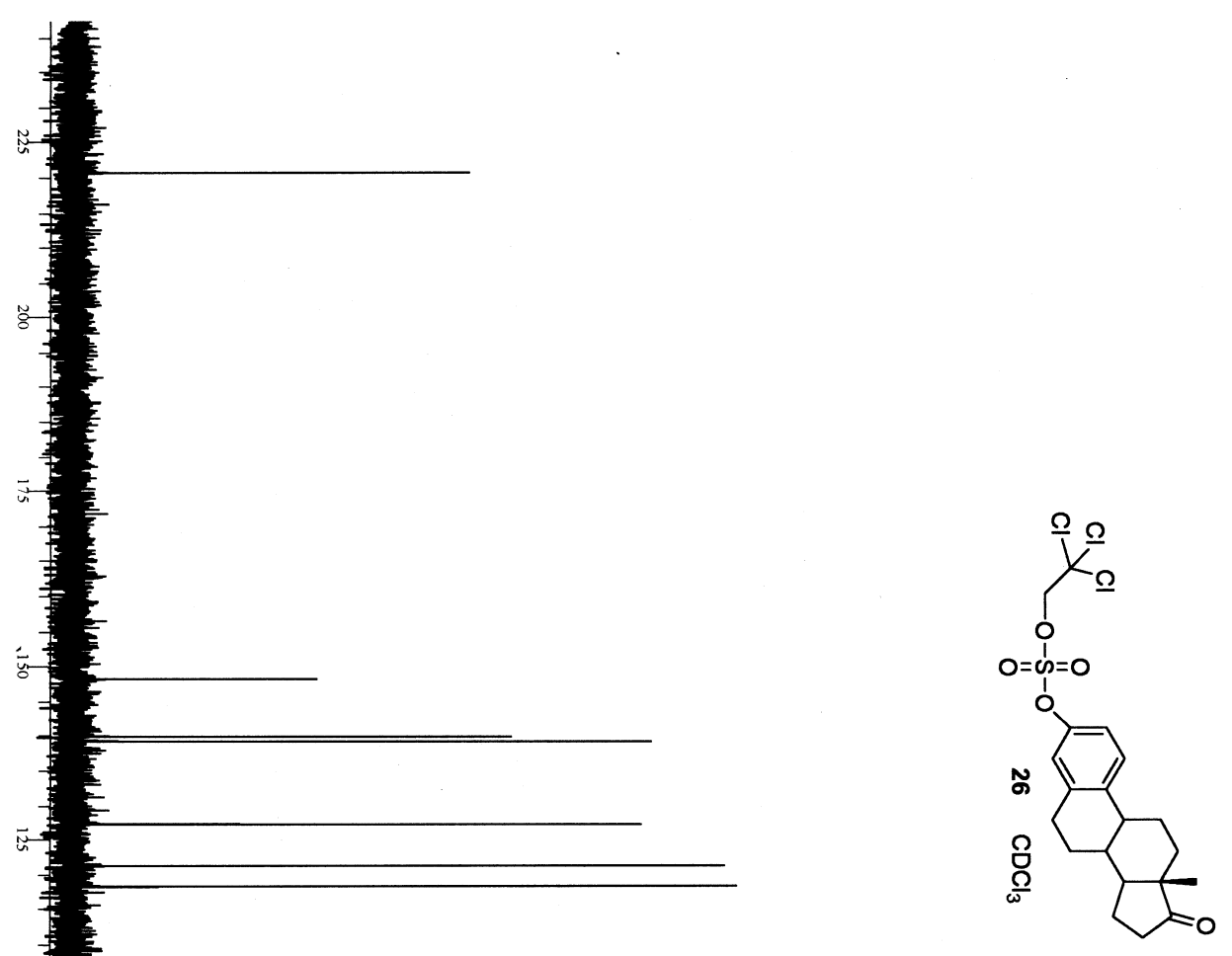




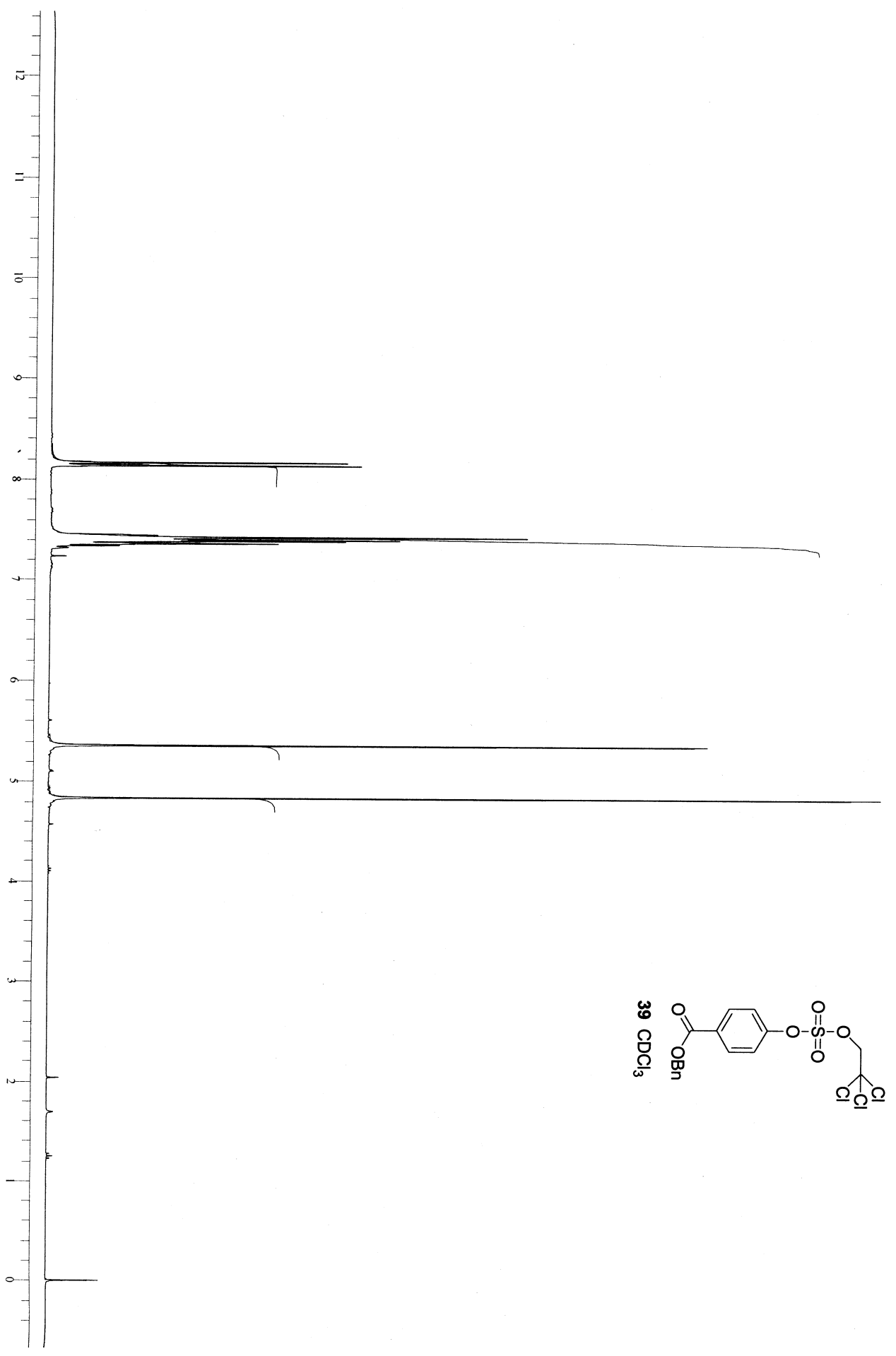




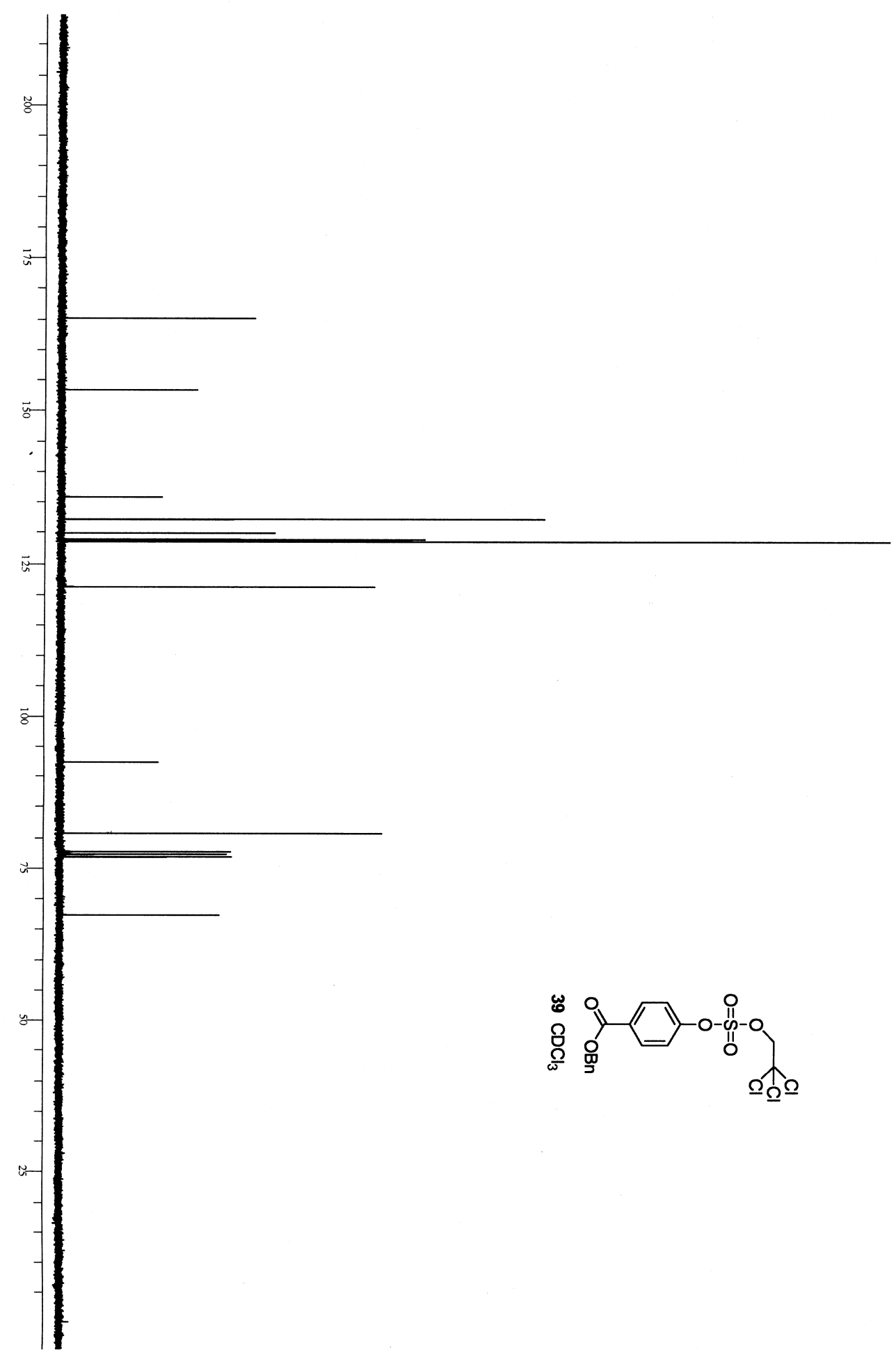




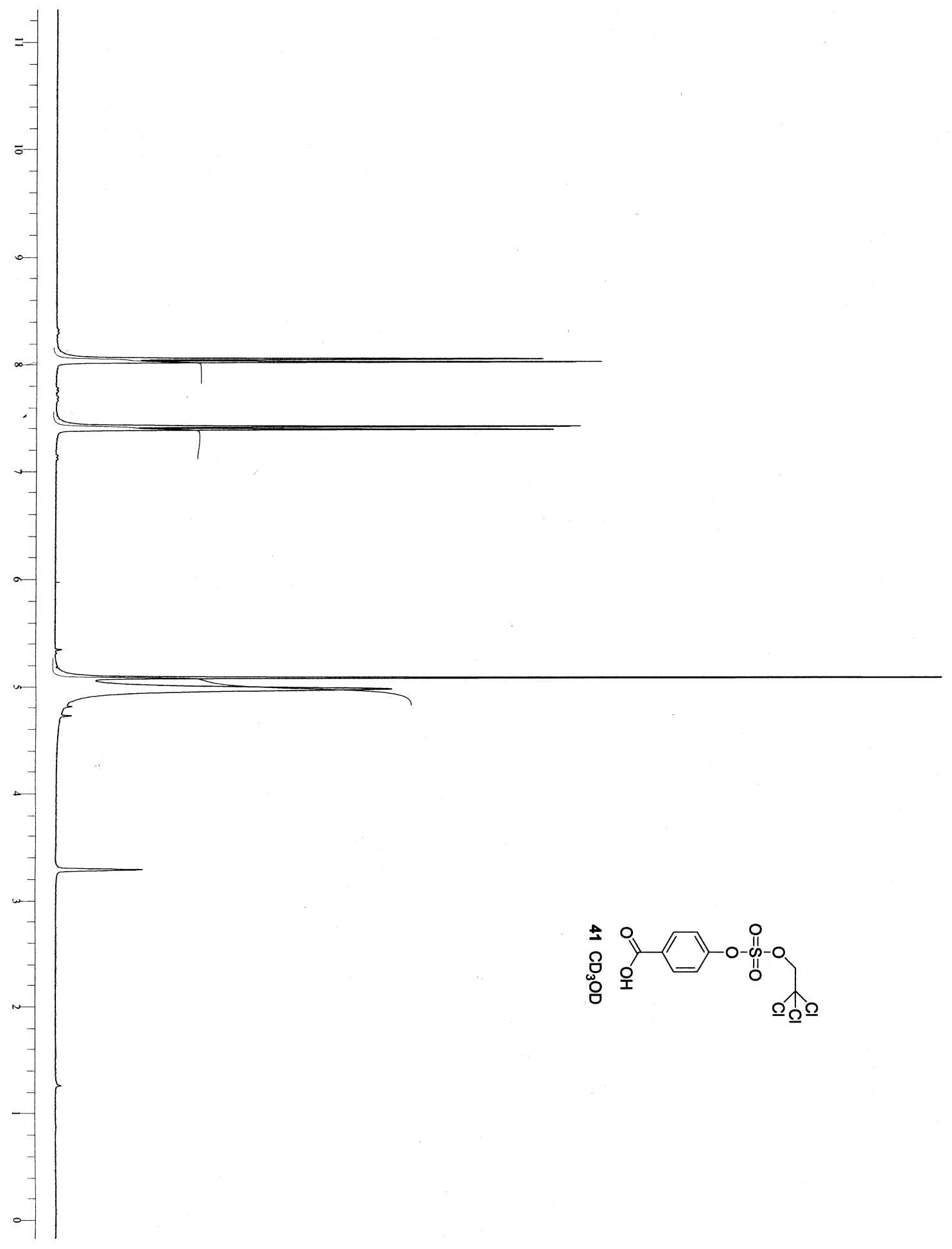




$$
E
$$




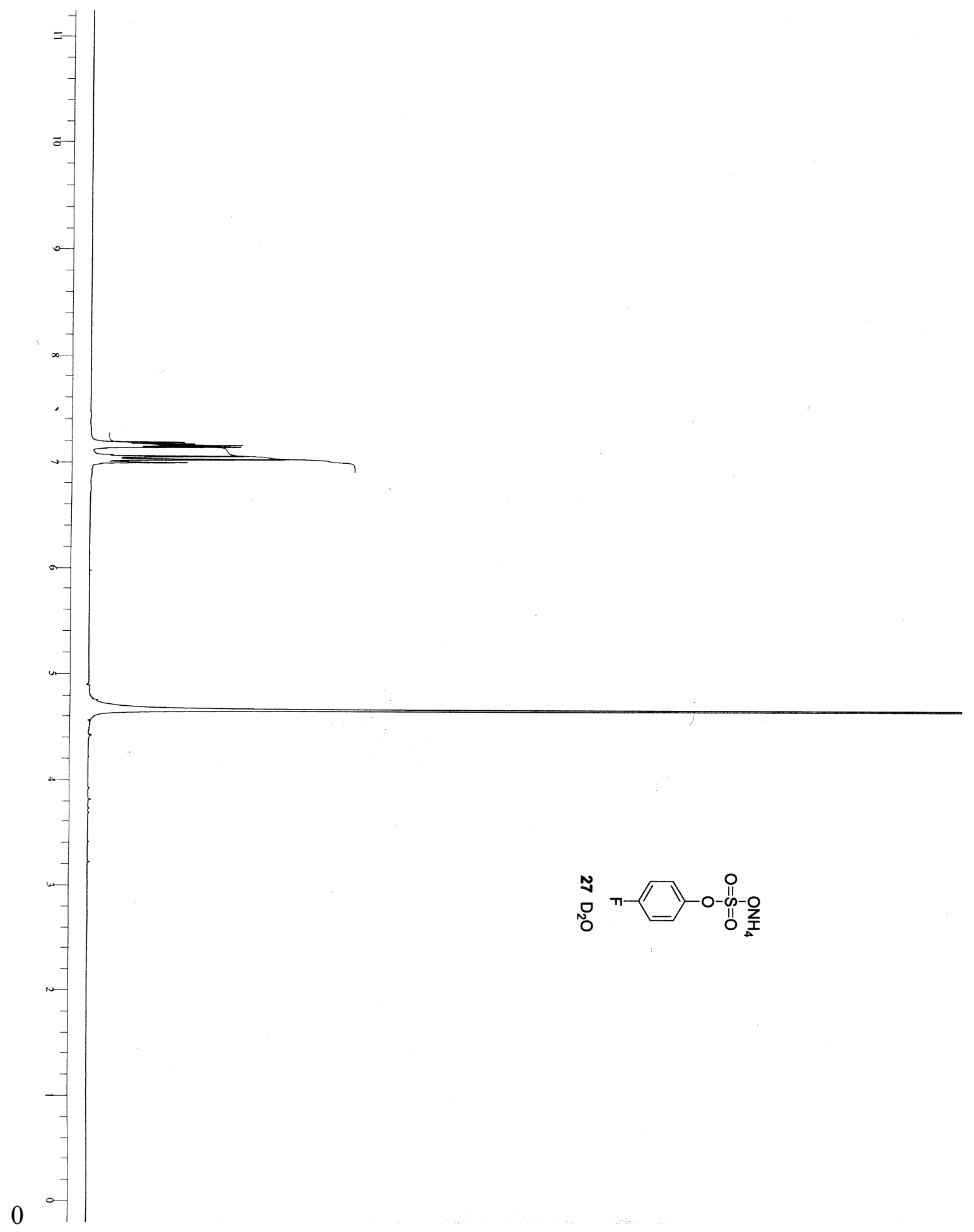




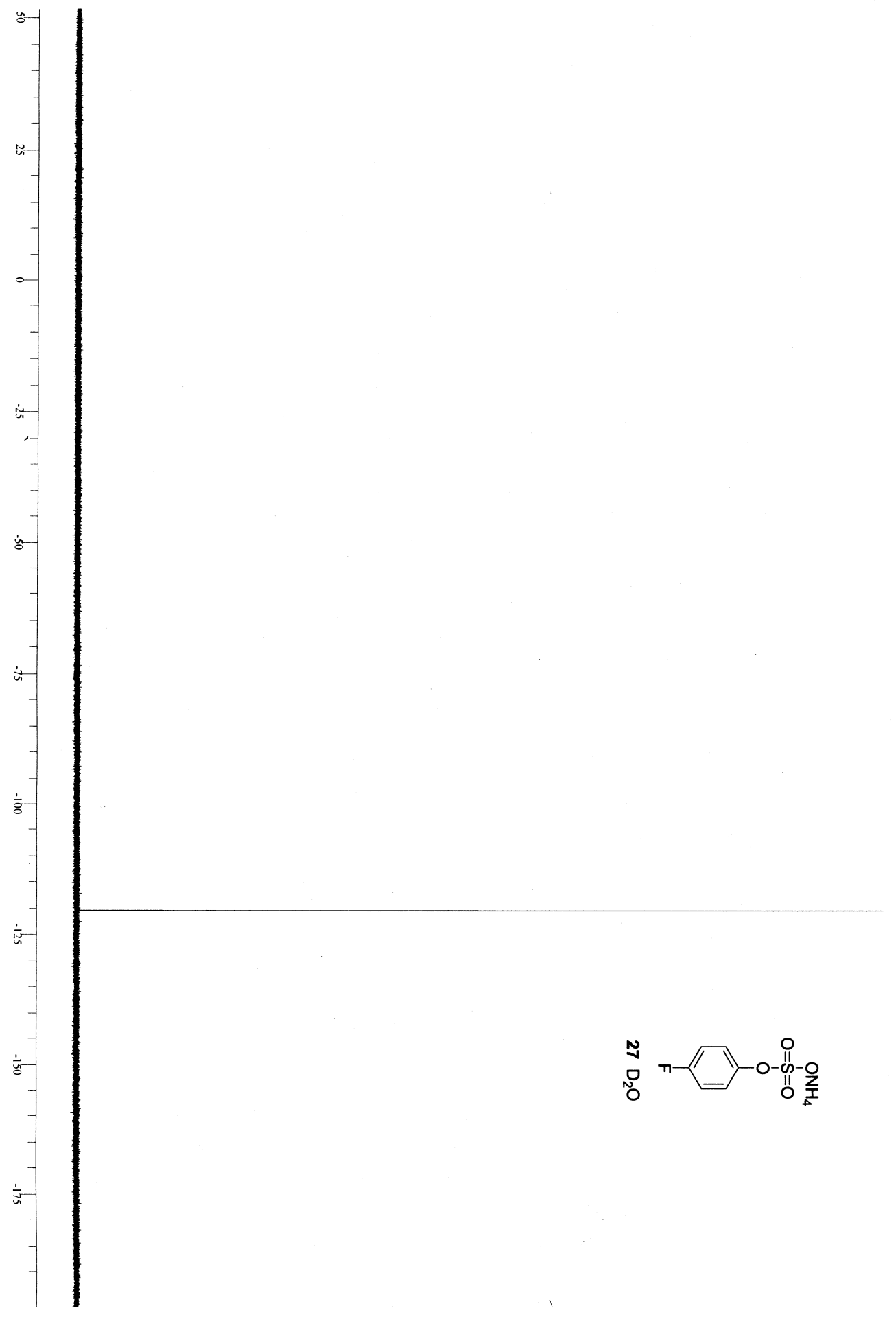




$$
\mid
$$




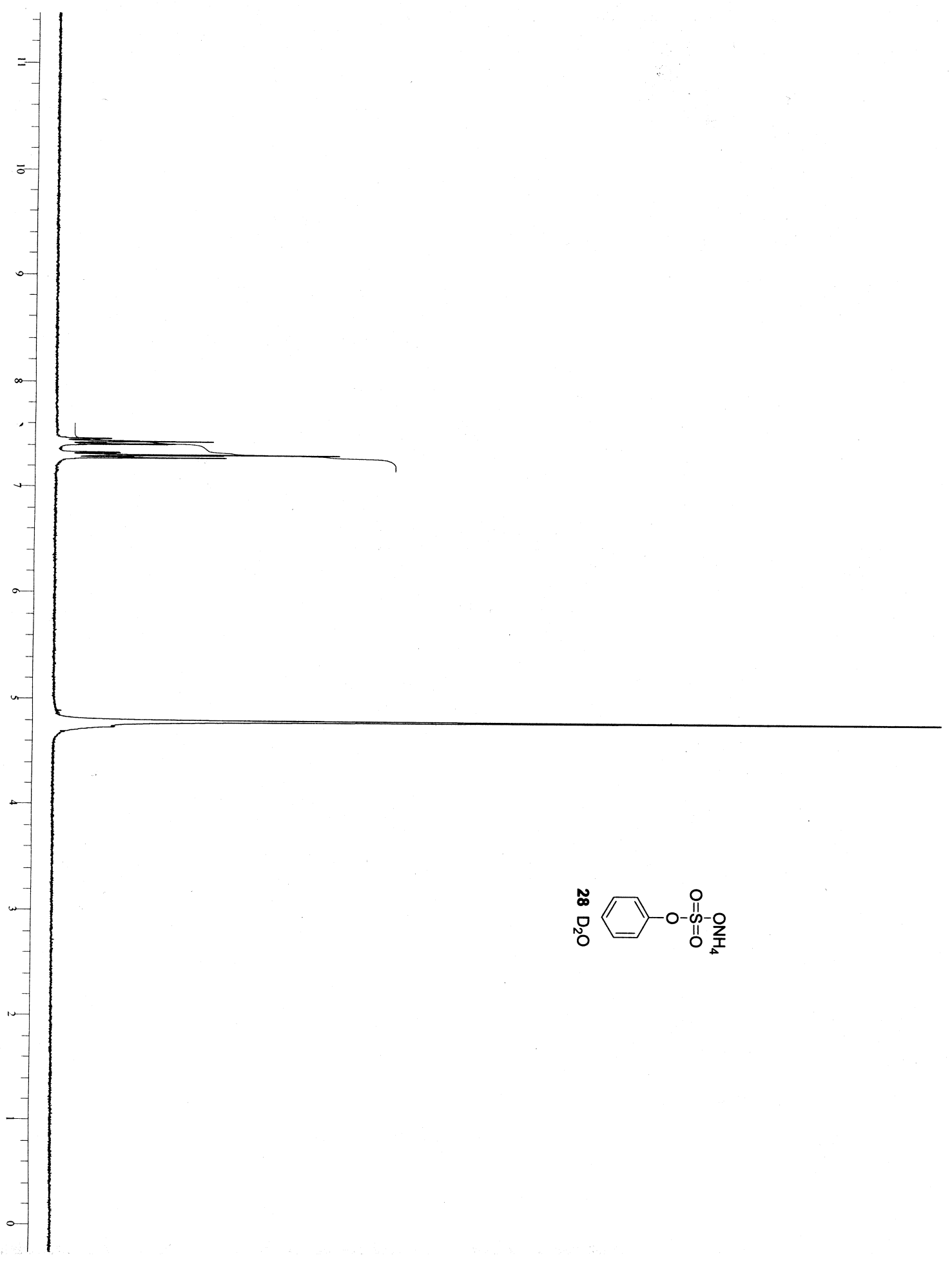




$$
\mid
$$




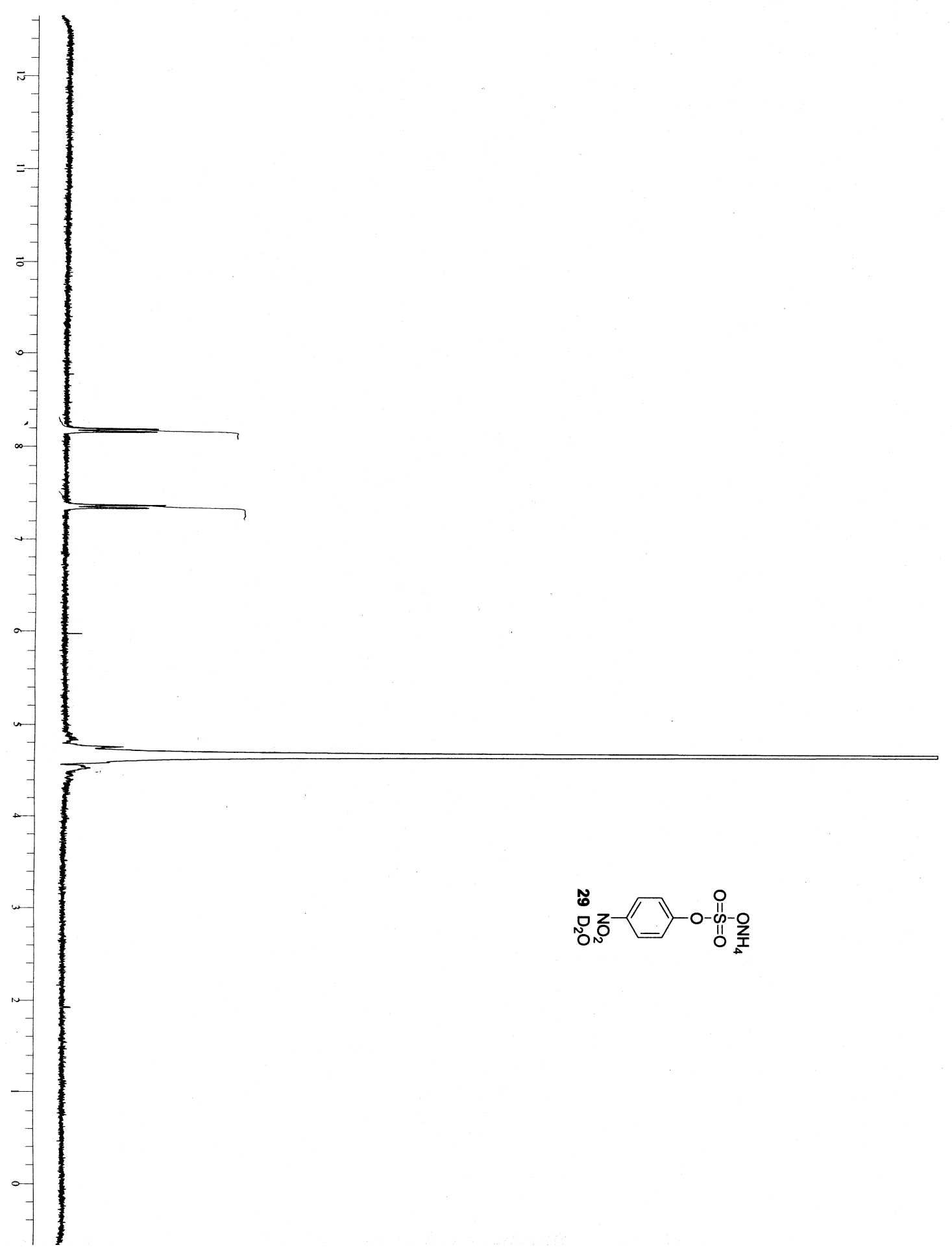




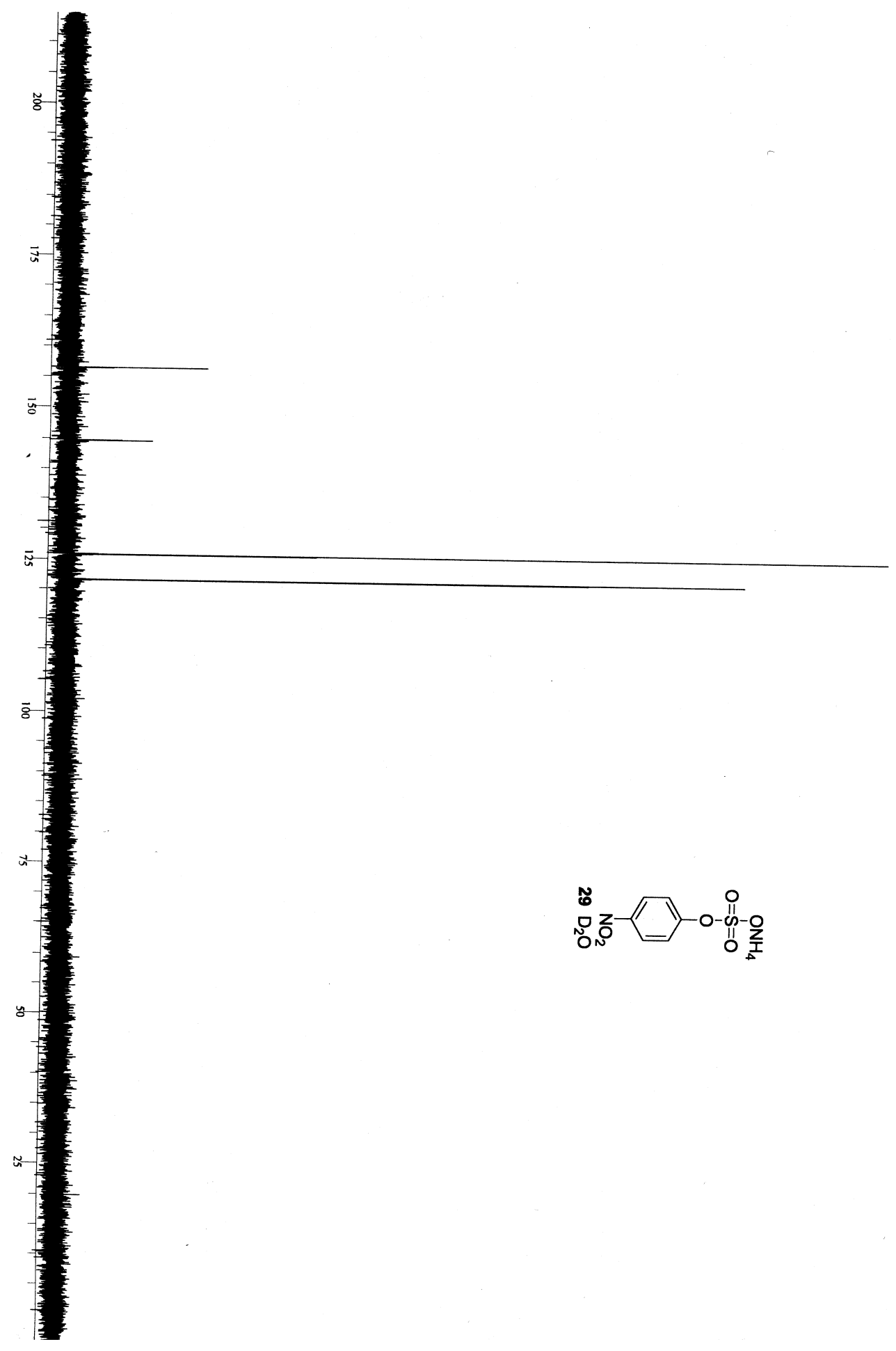




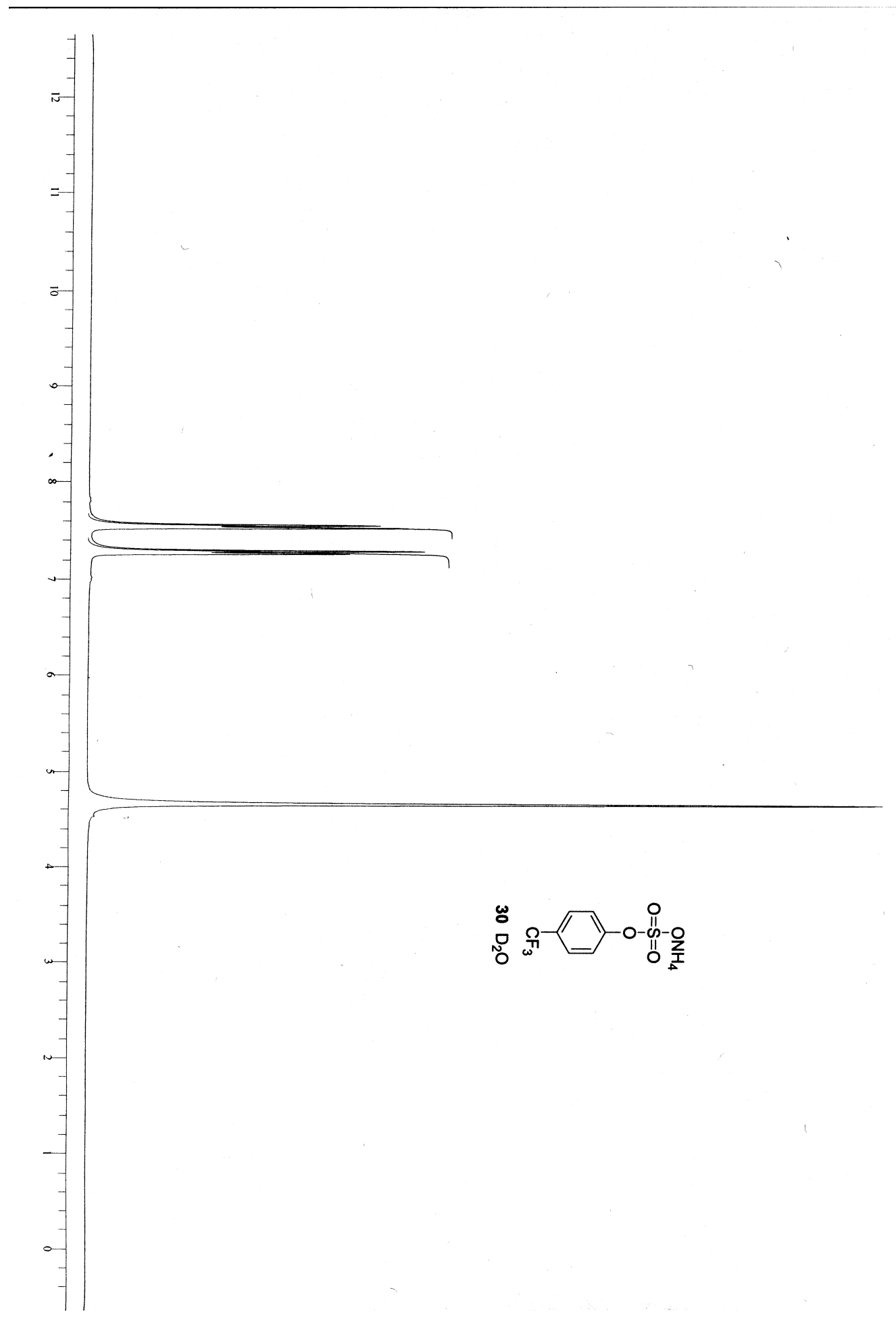



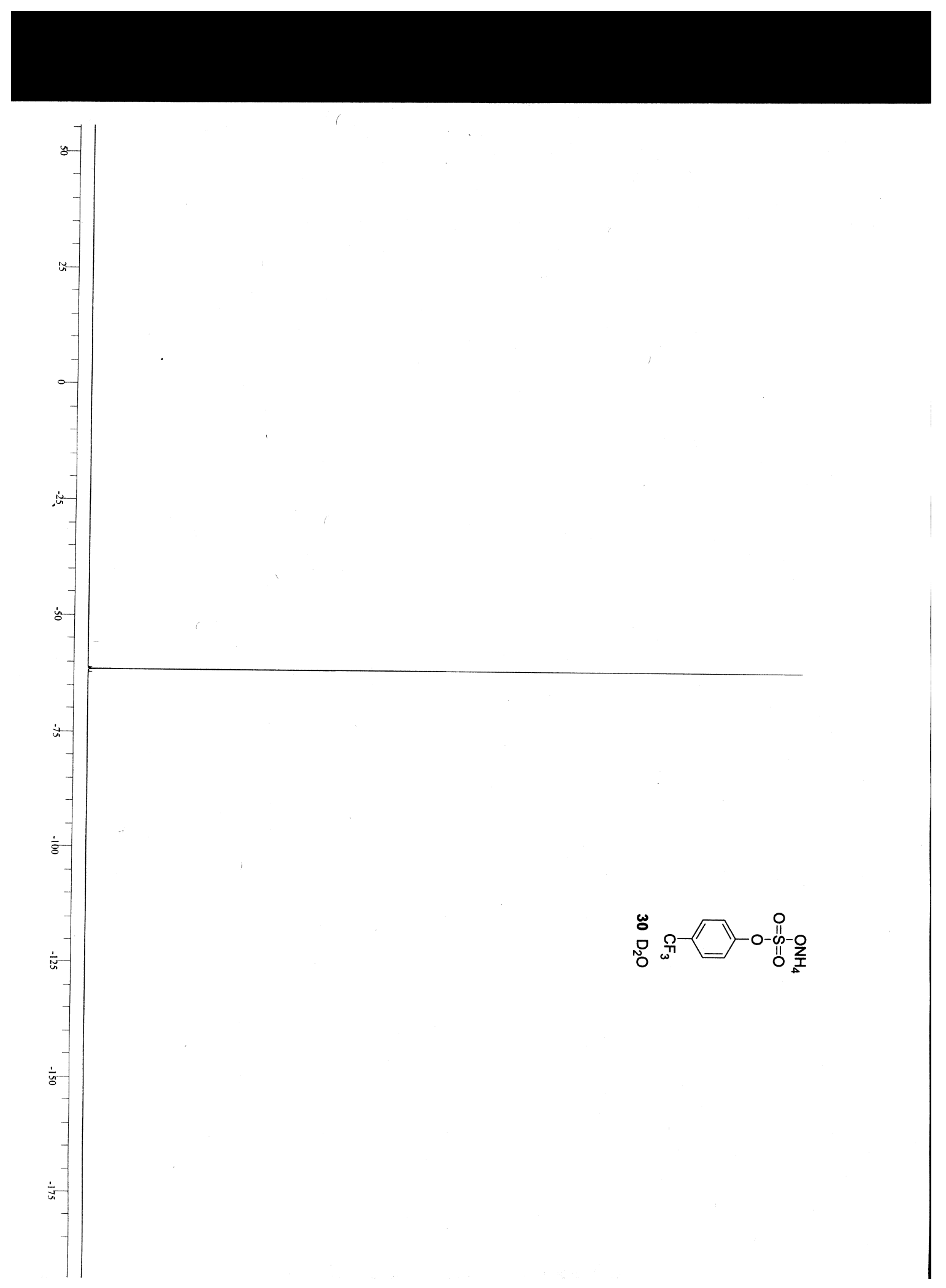


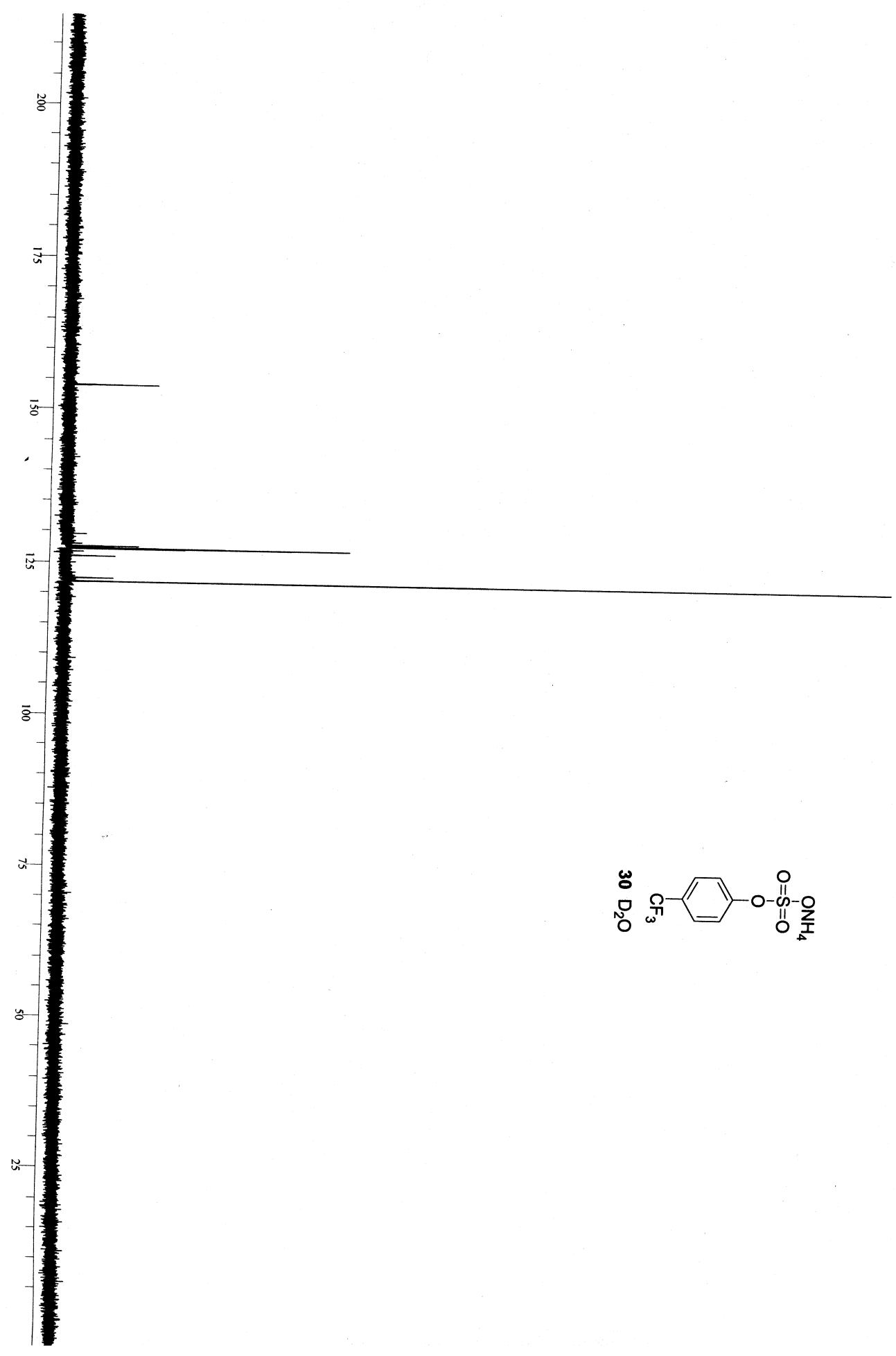




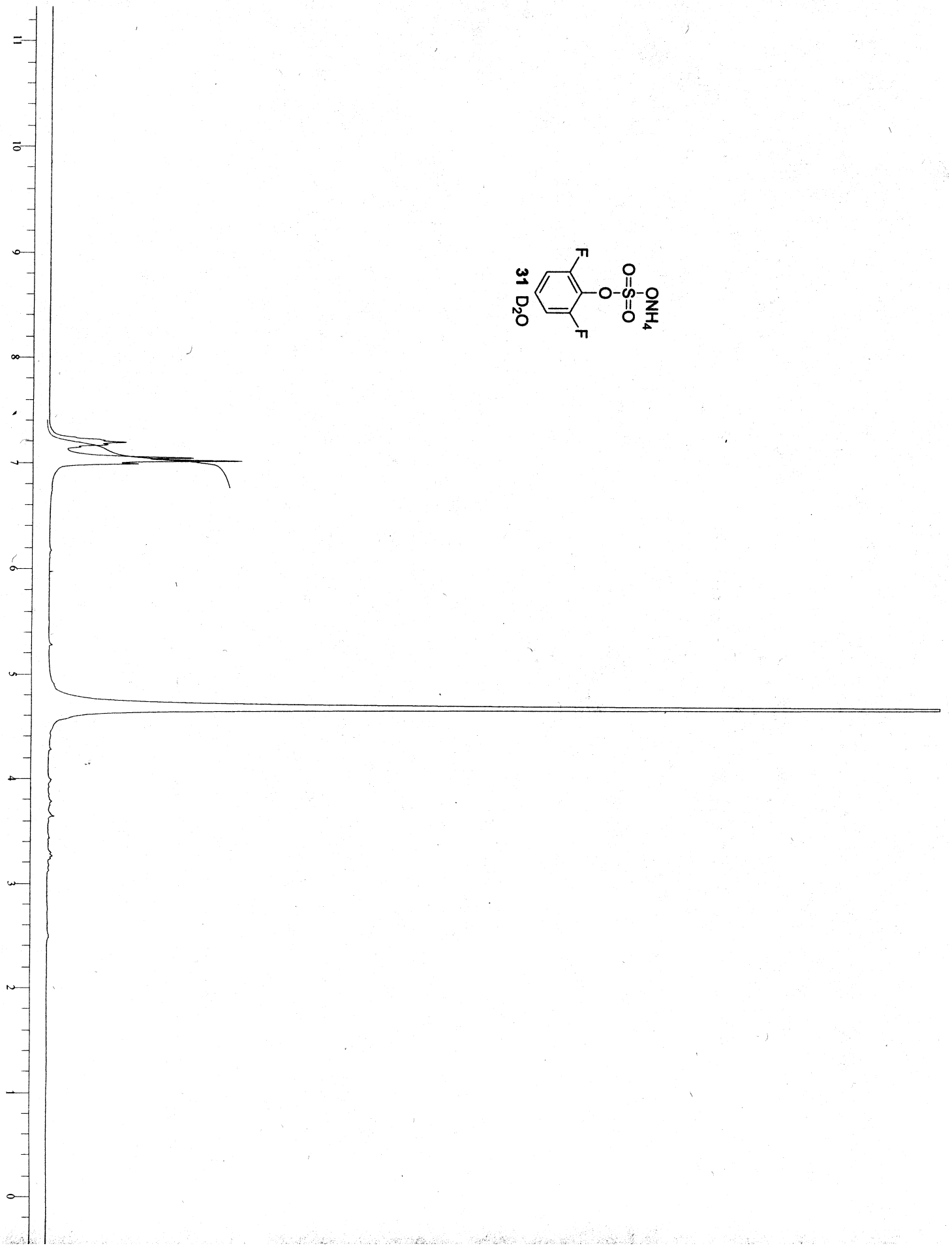




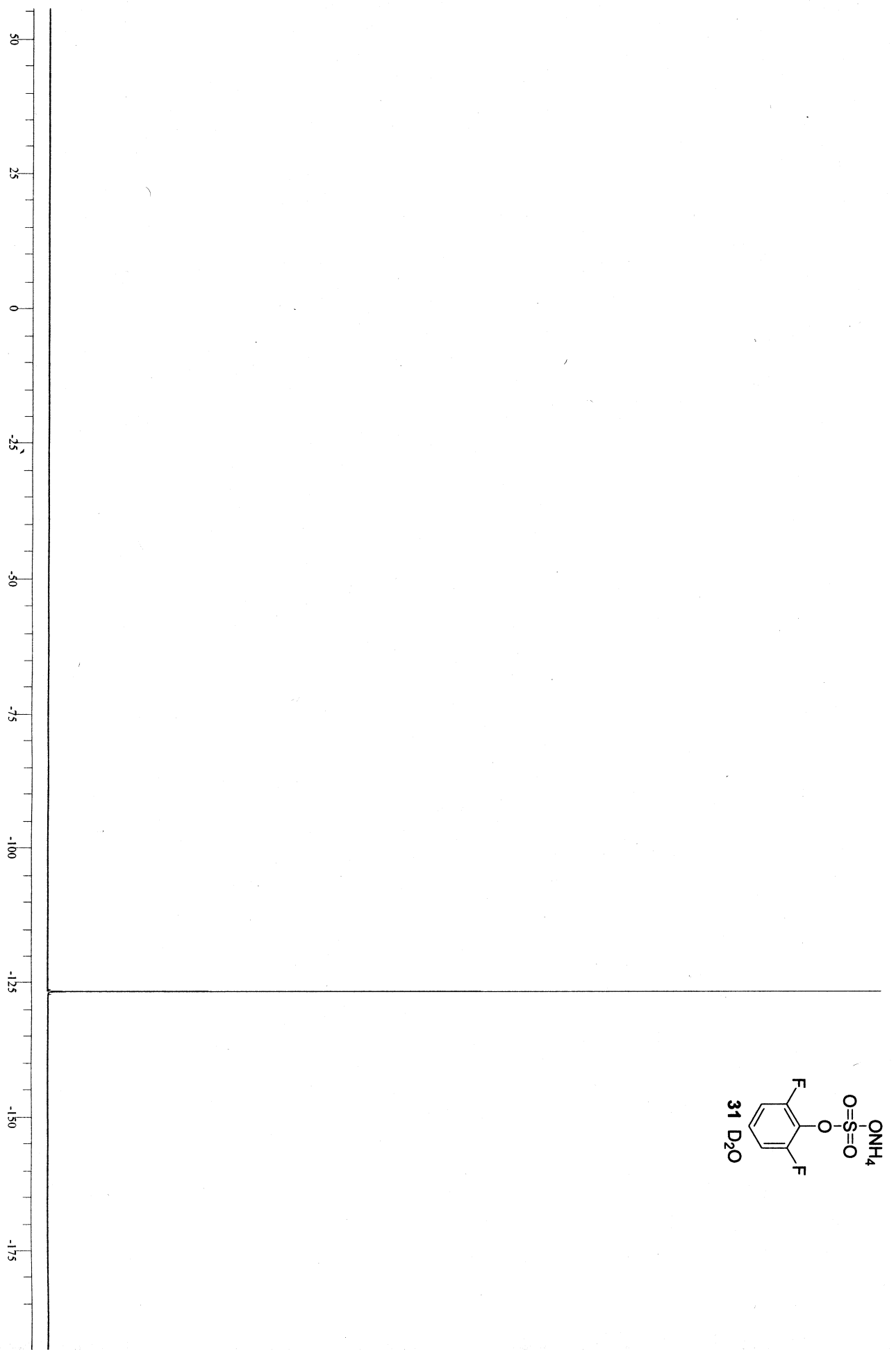




$$
\mid
$$




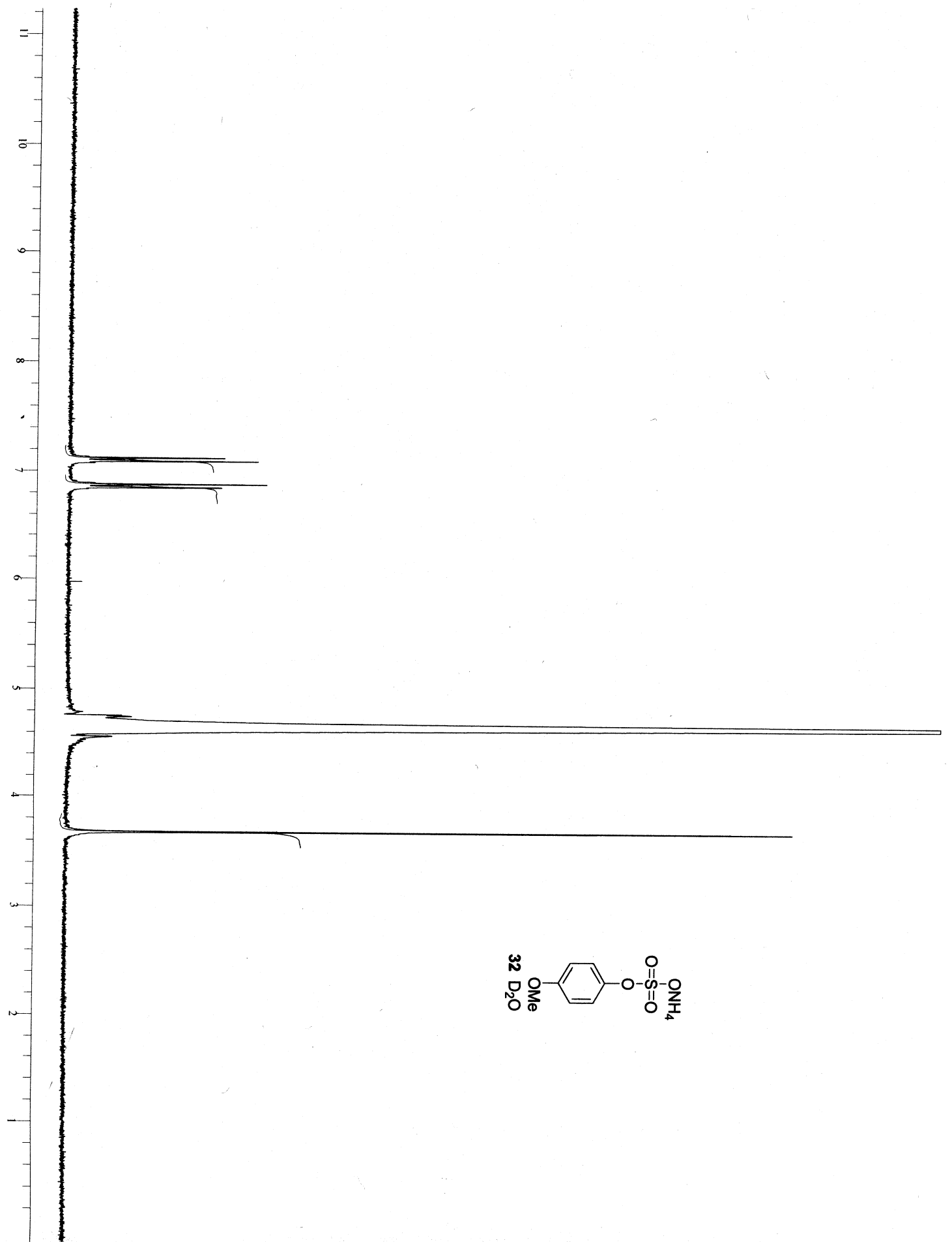




$$
\mid
$$




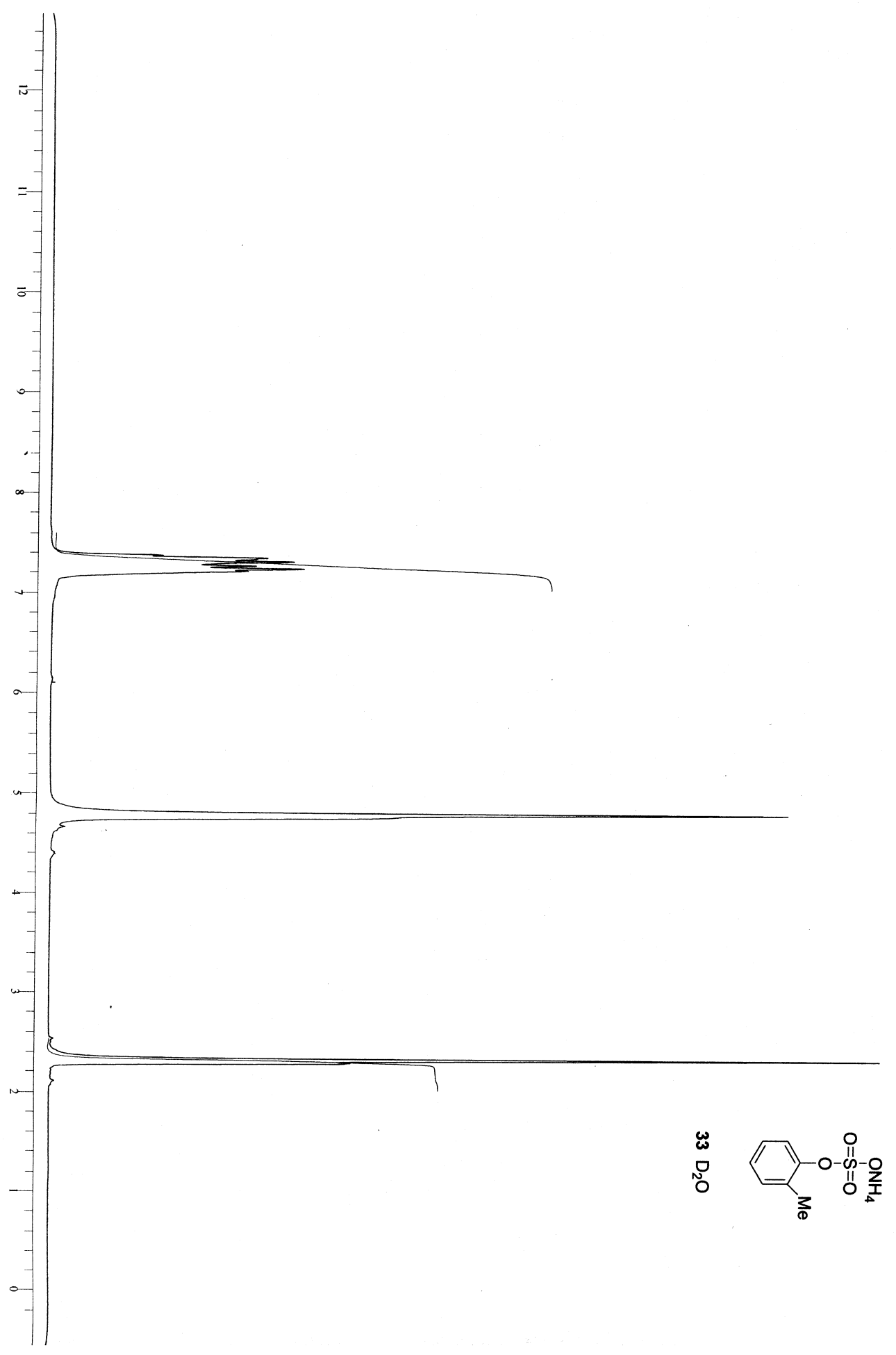




$$
F
$$




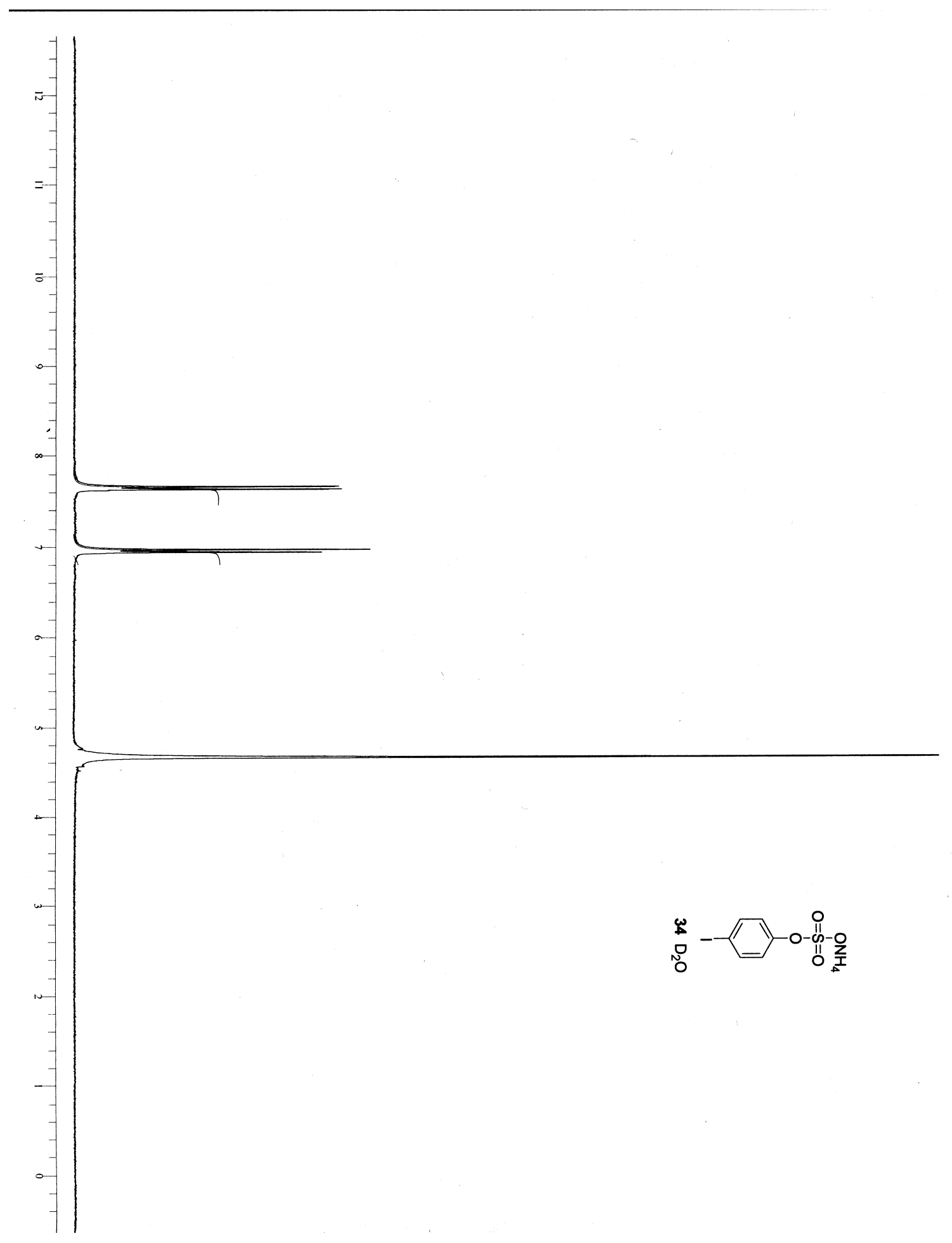




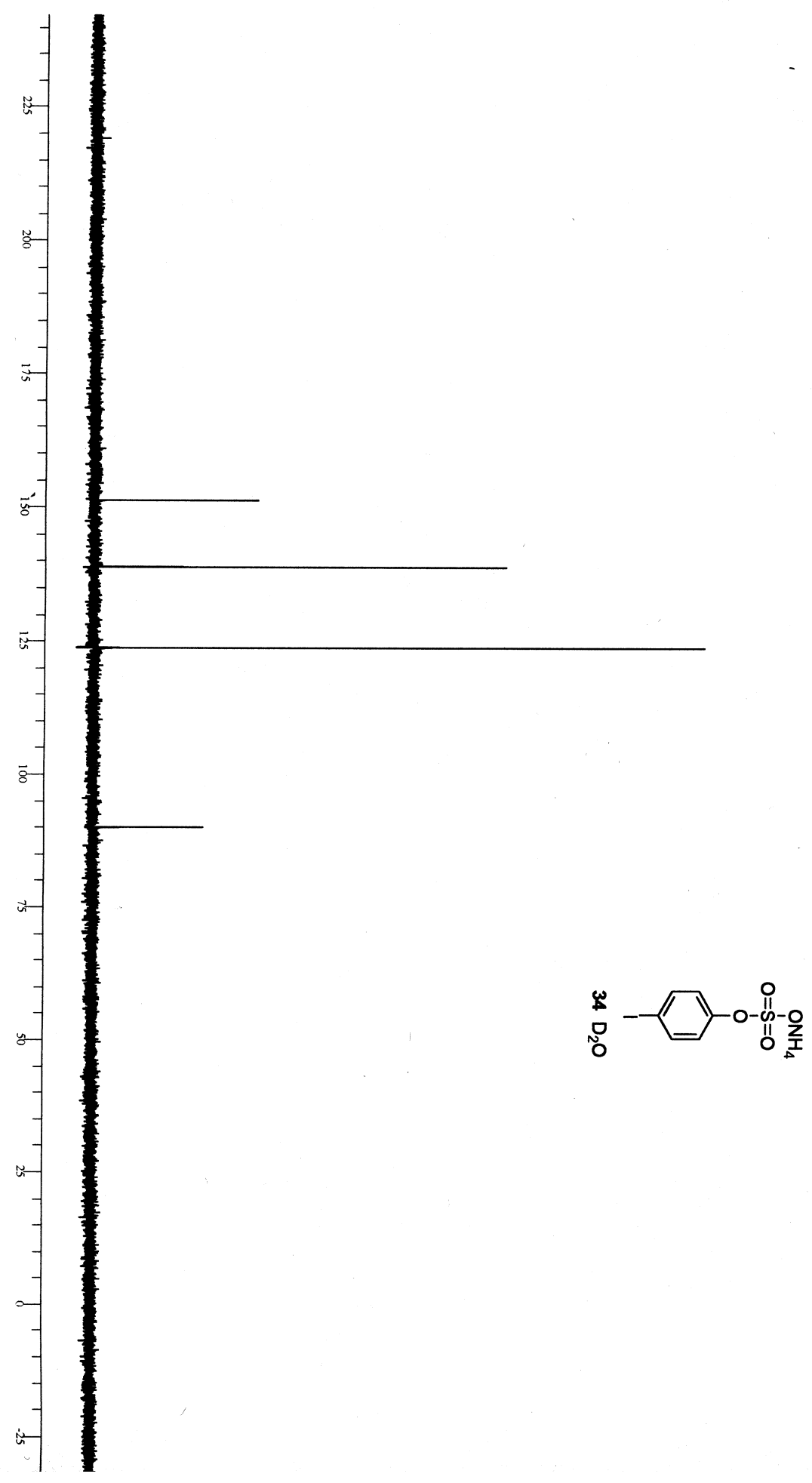




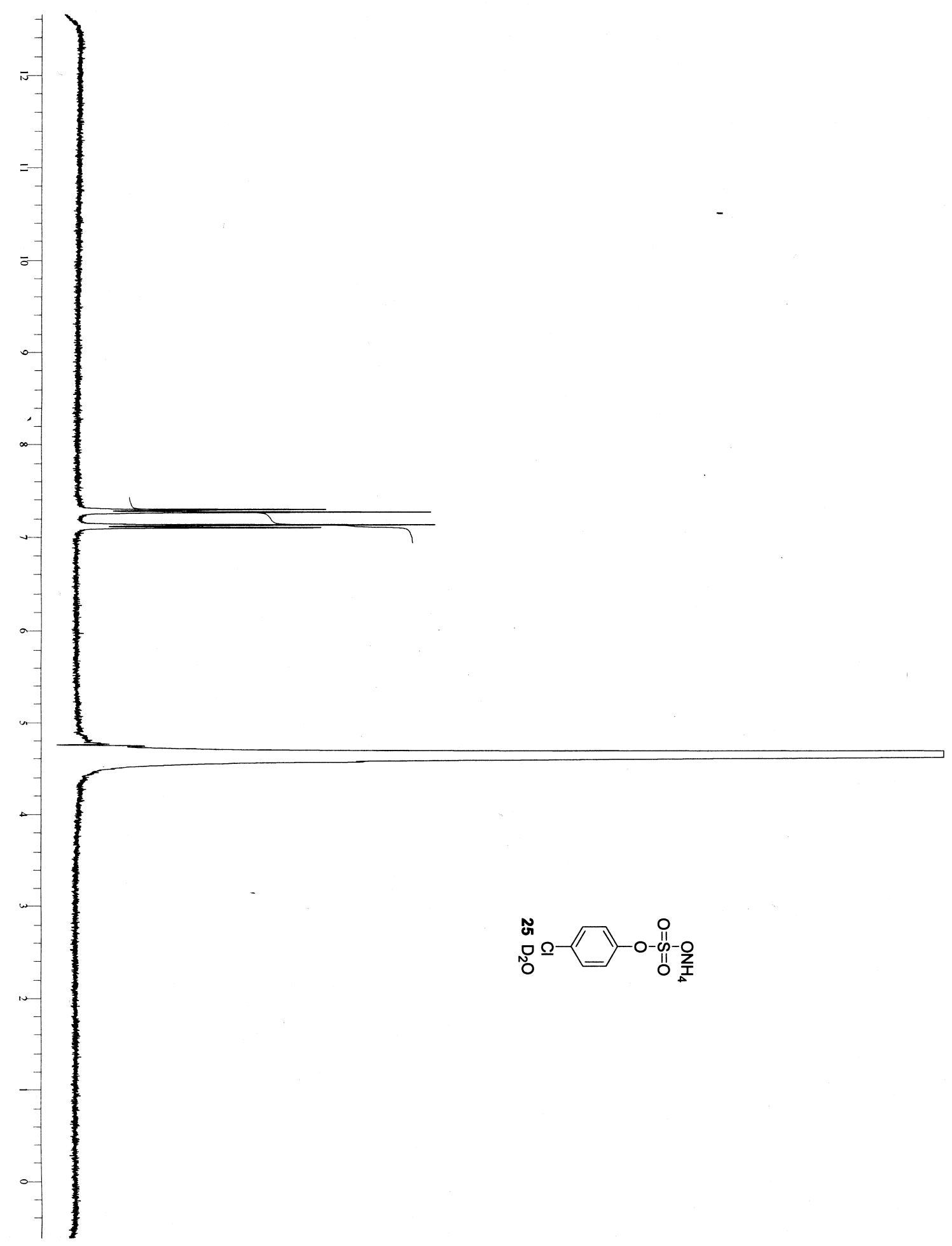




$$
\mid
$$




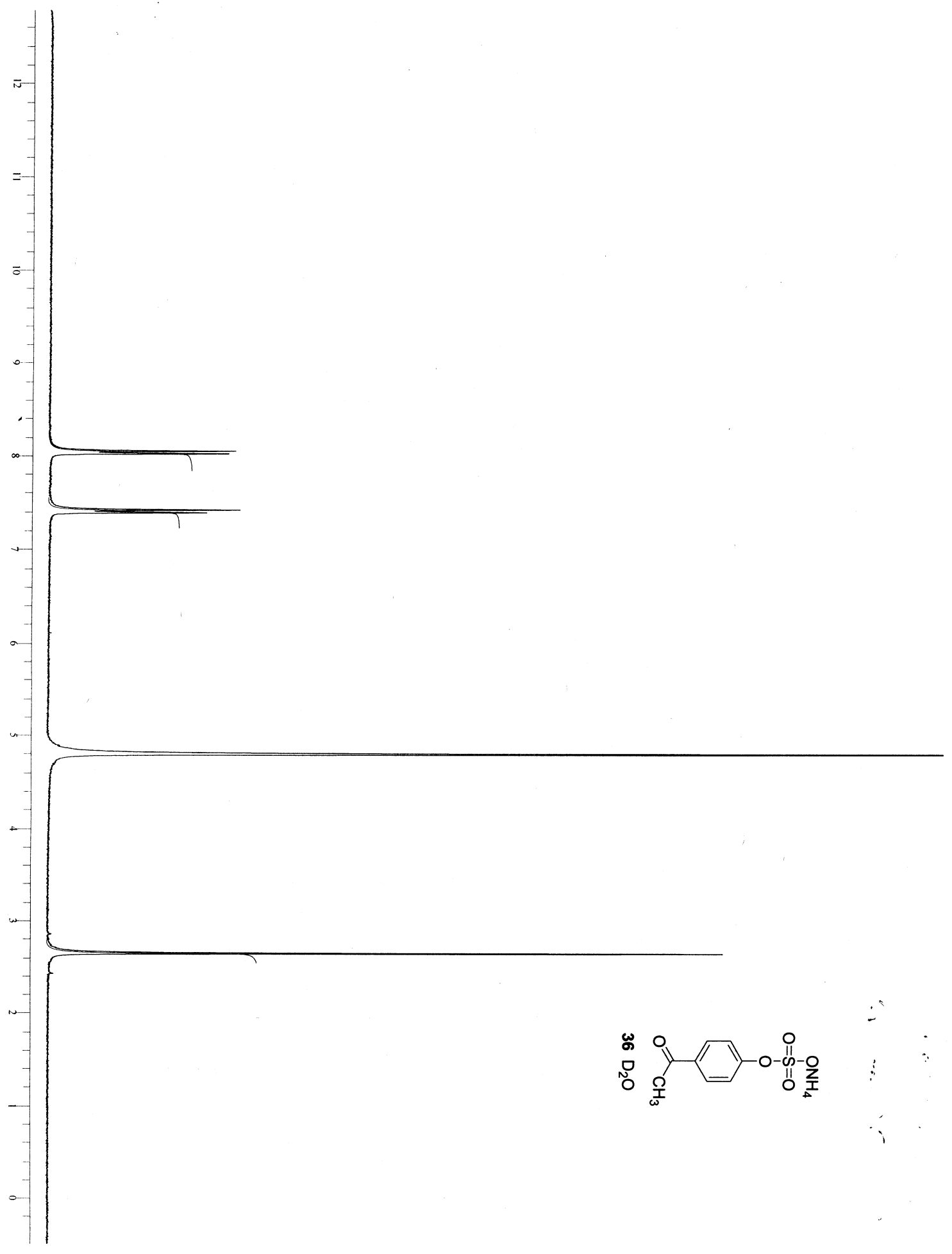




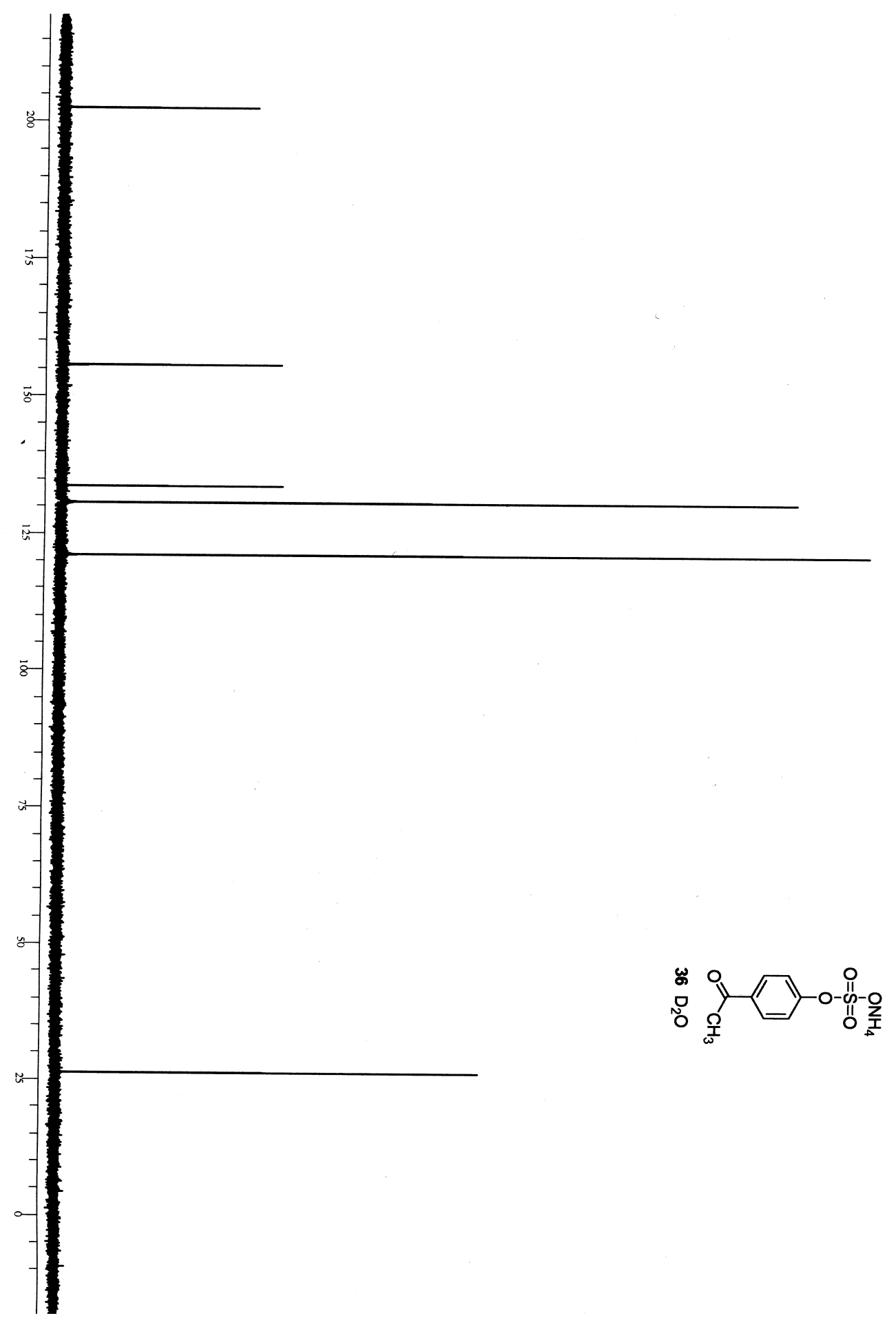




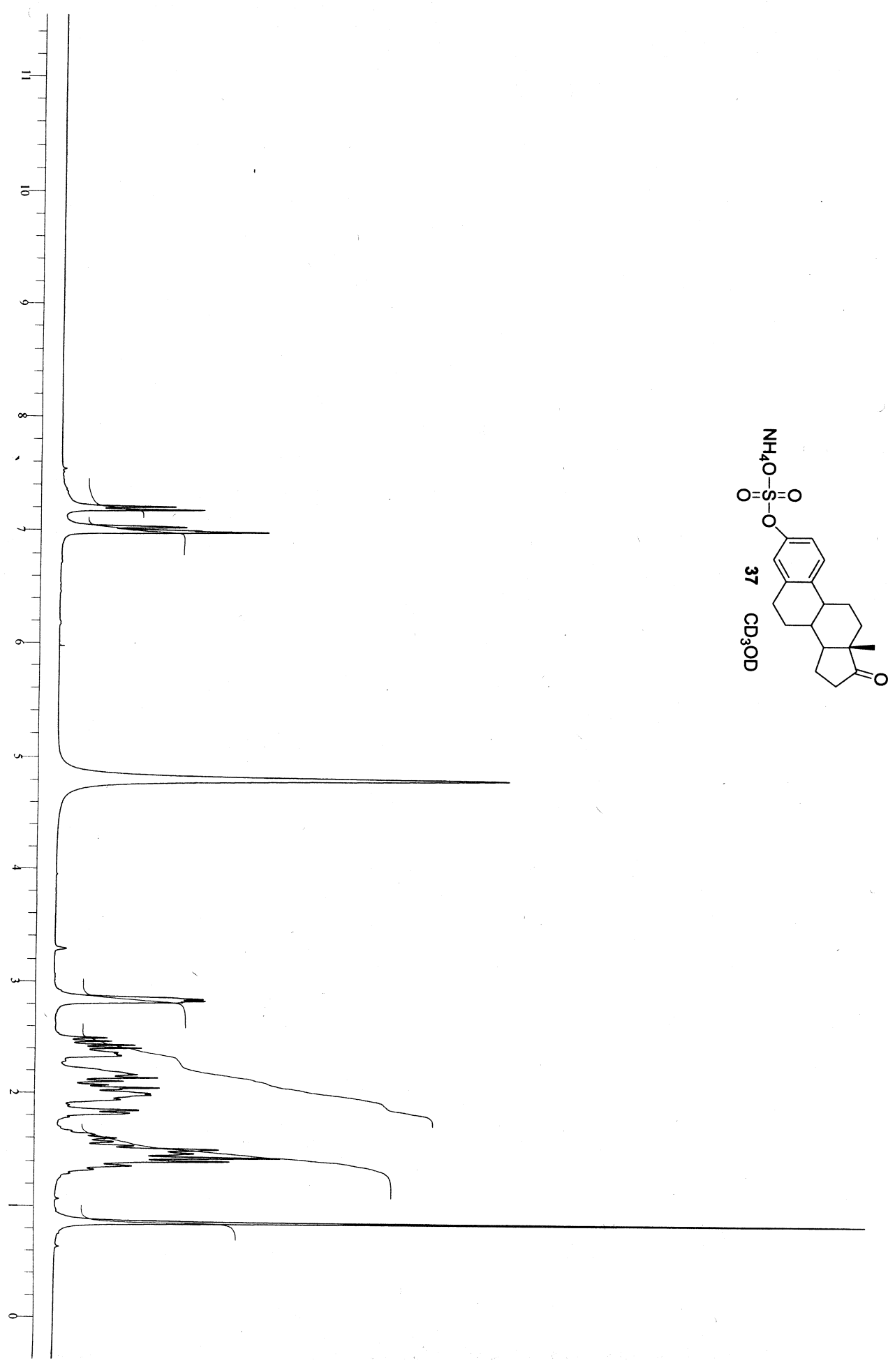




$$
F
$$




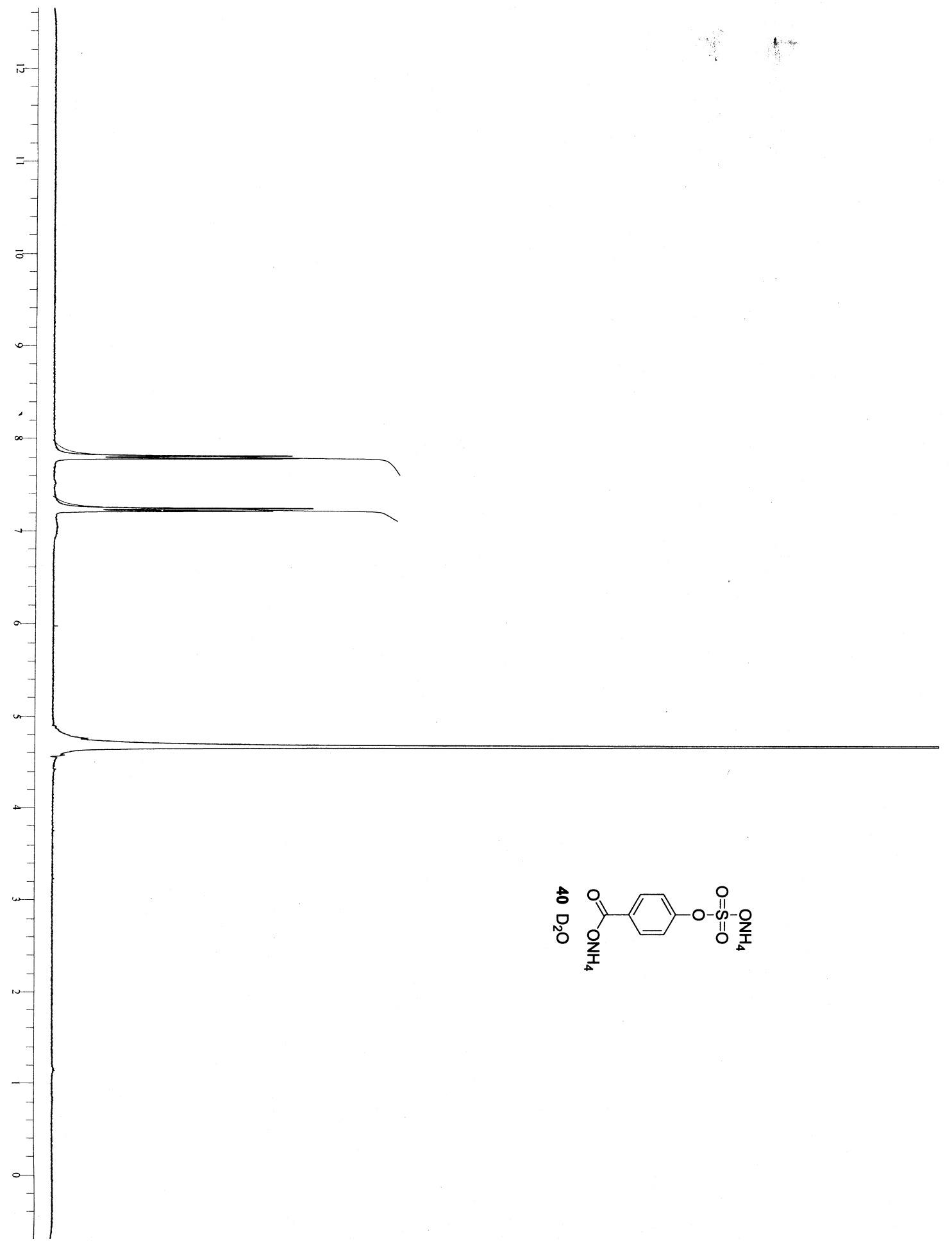




$$
\mid
$$




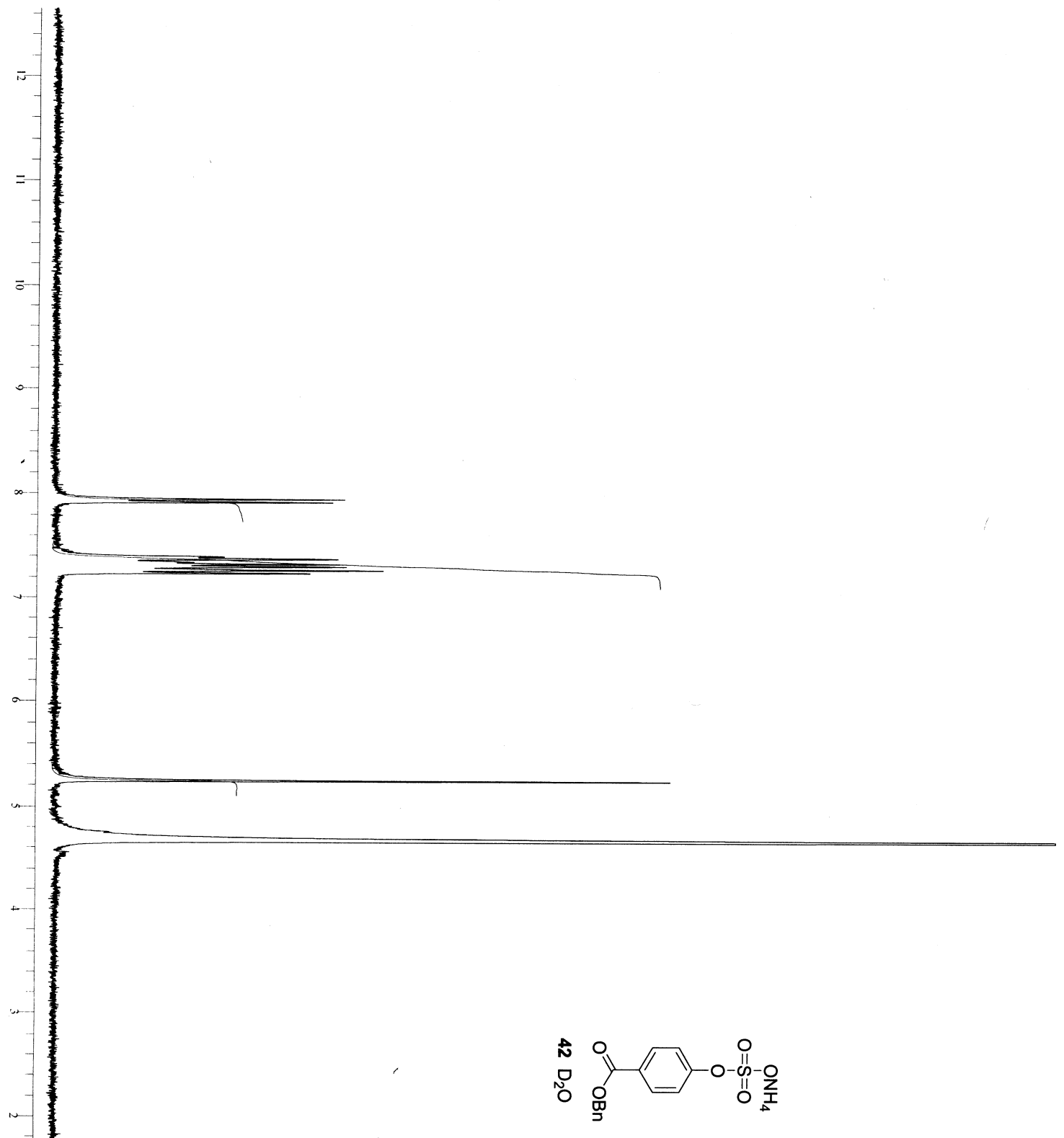

66 


$$
F
$$




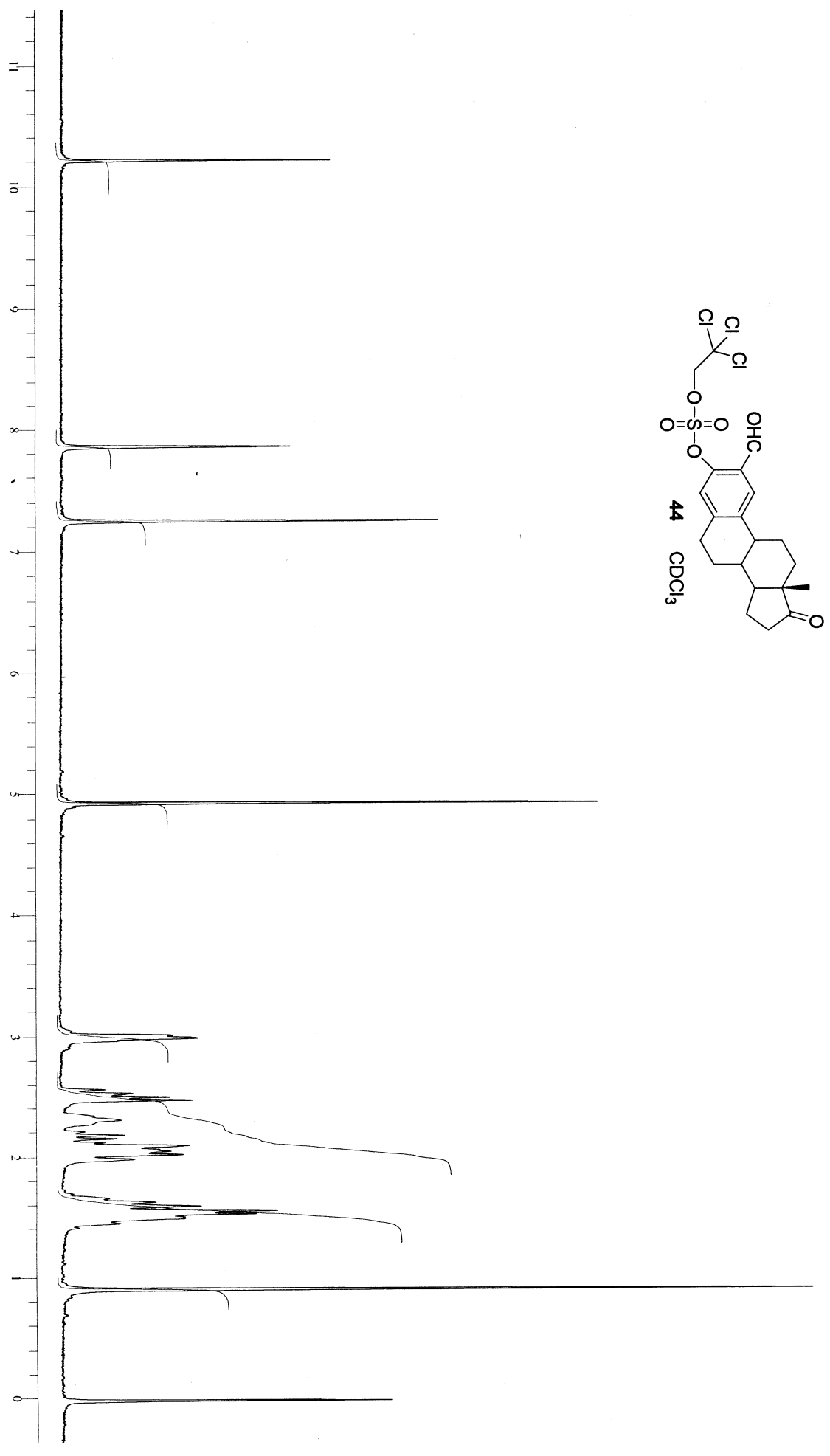




$$
E
$$




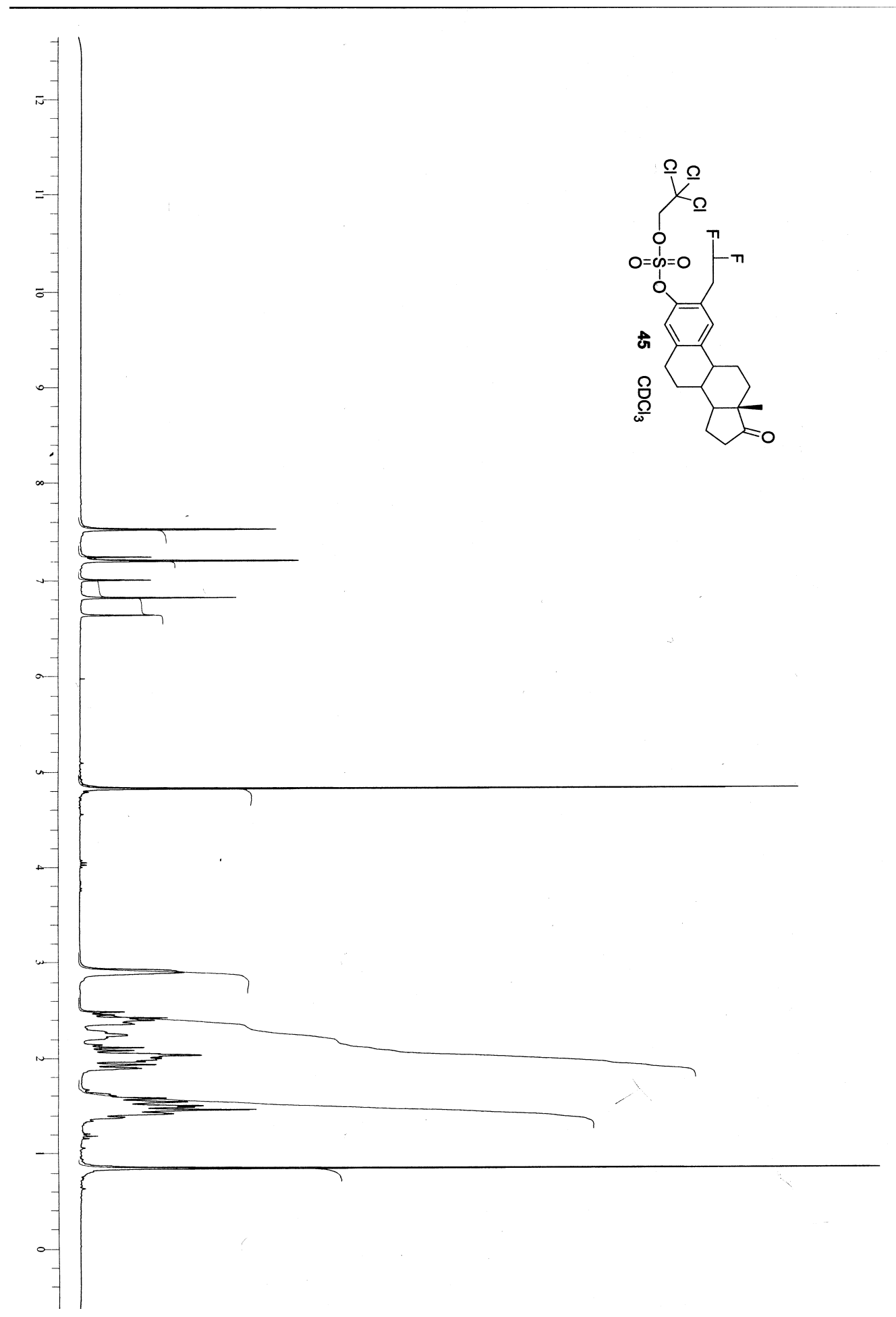



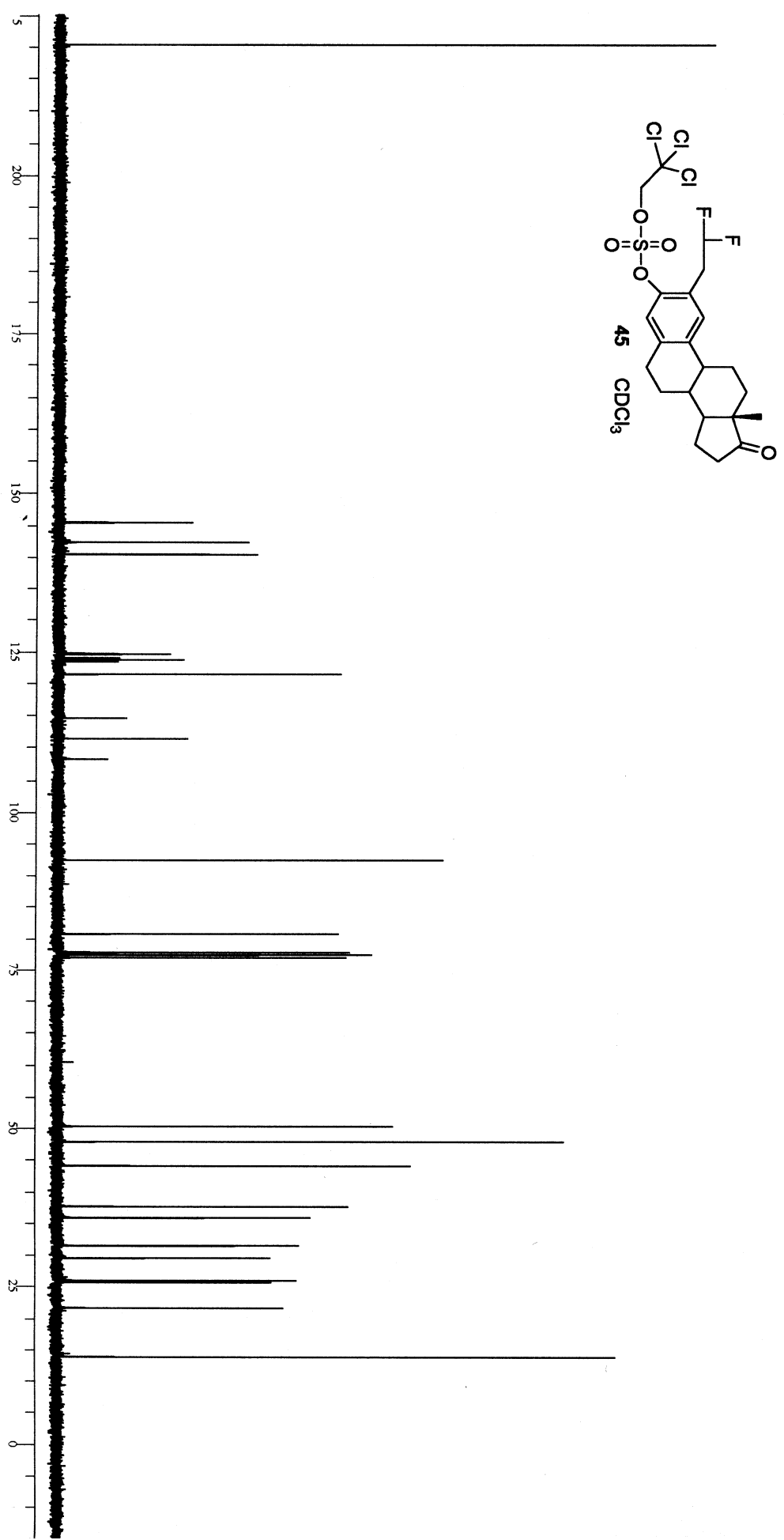


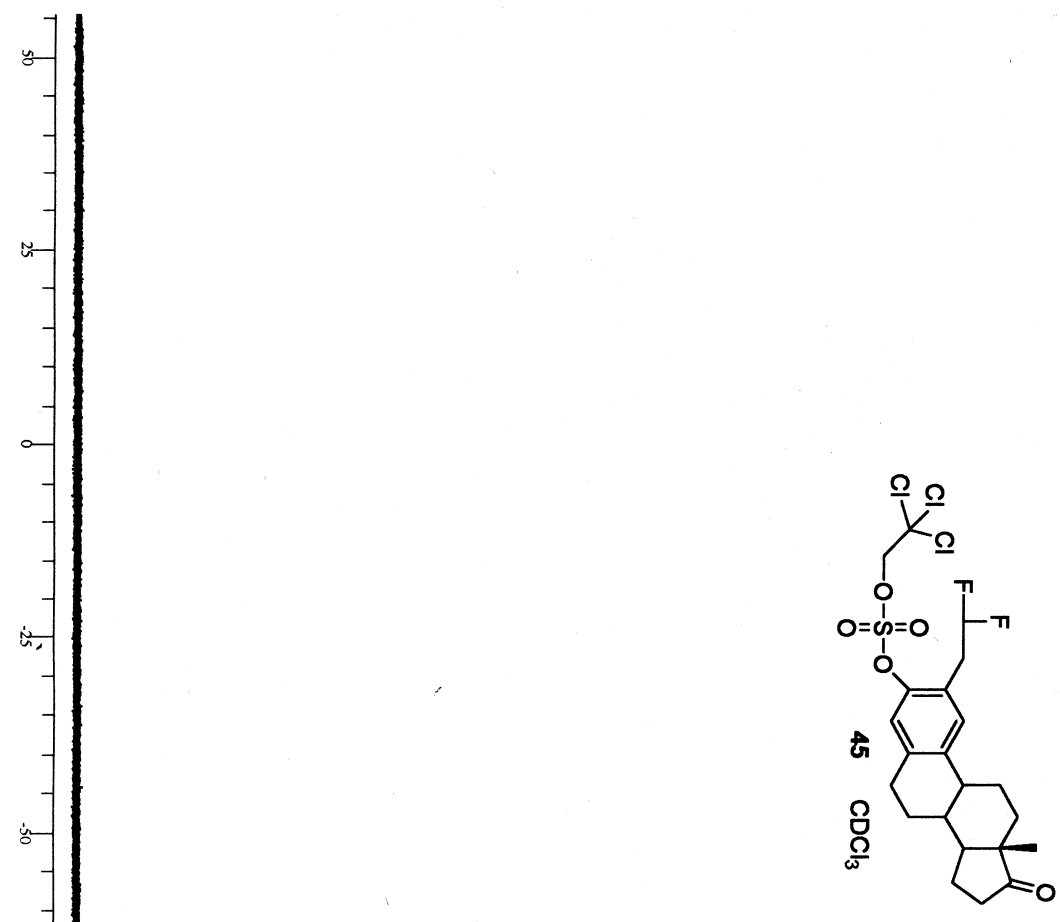

72 


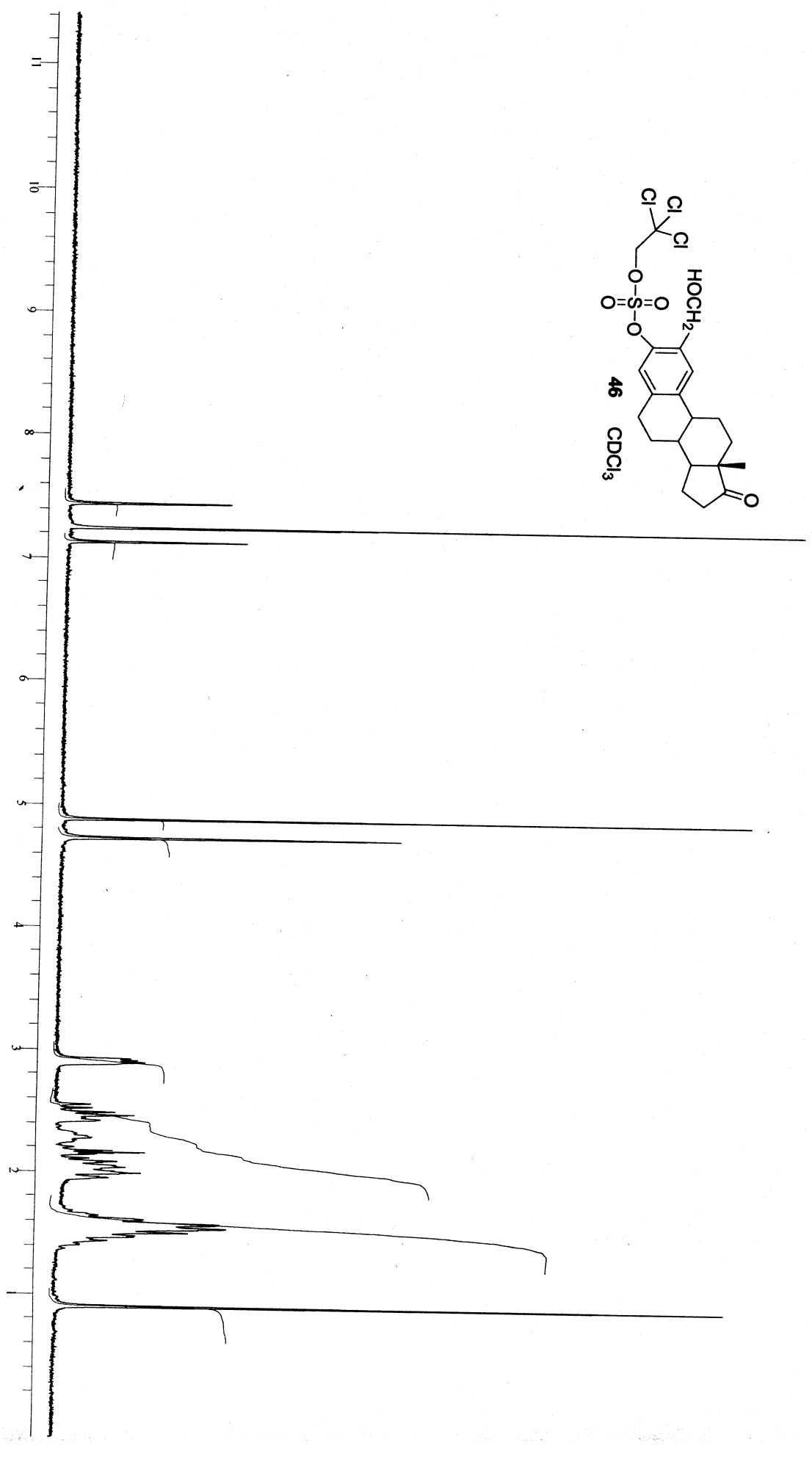

73 

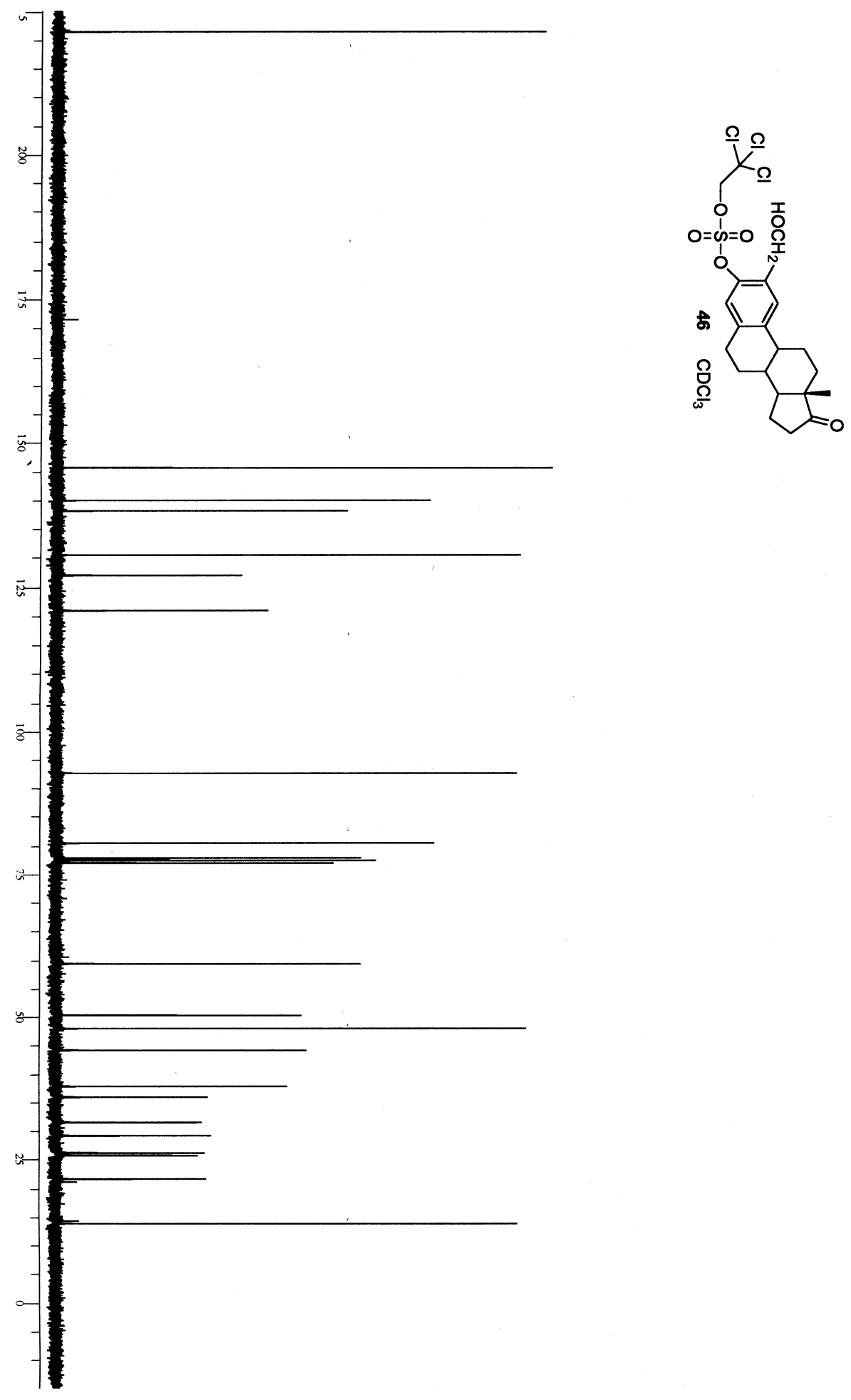


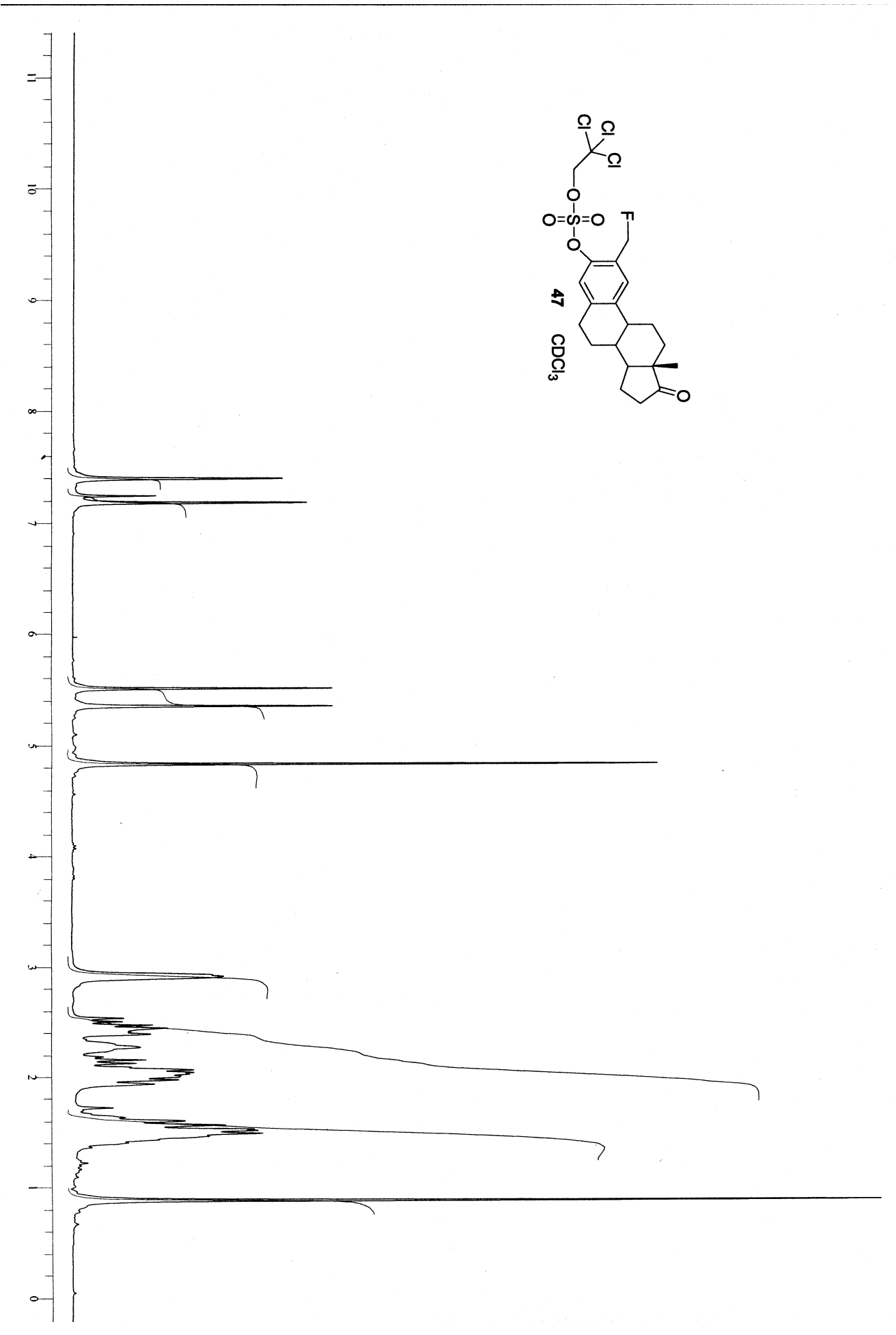




$$
\mid i
$$




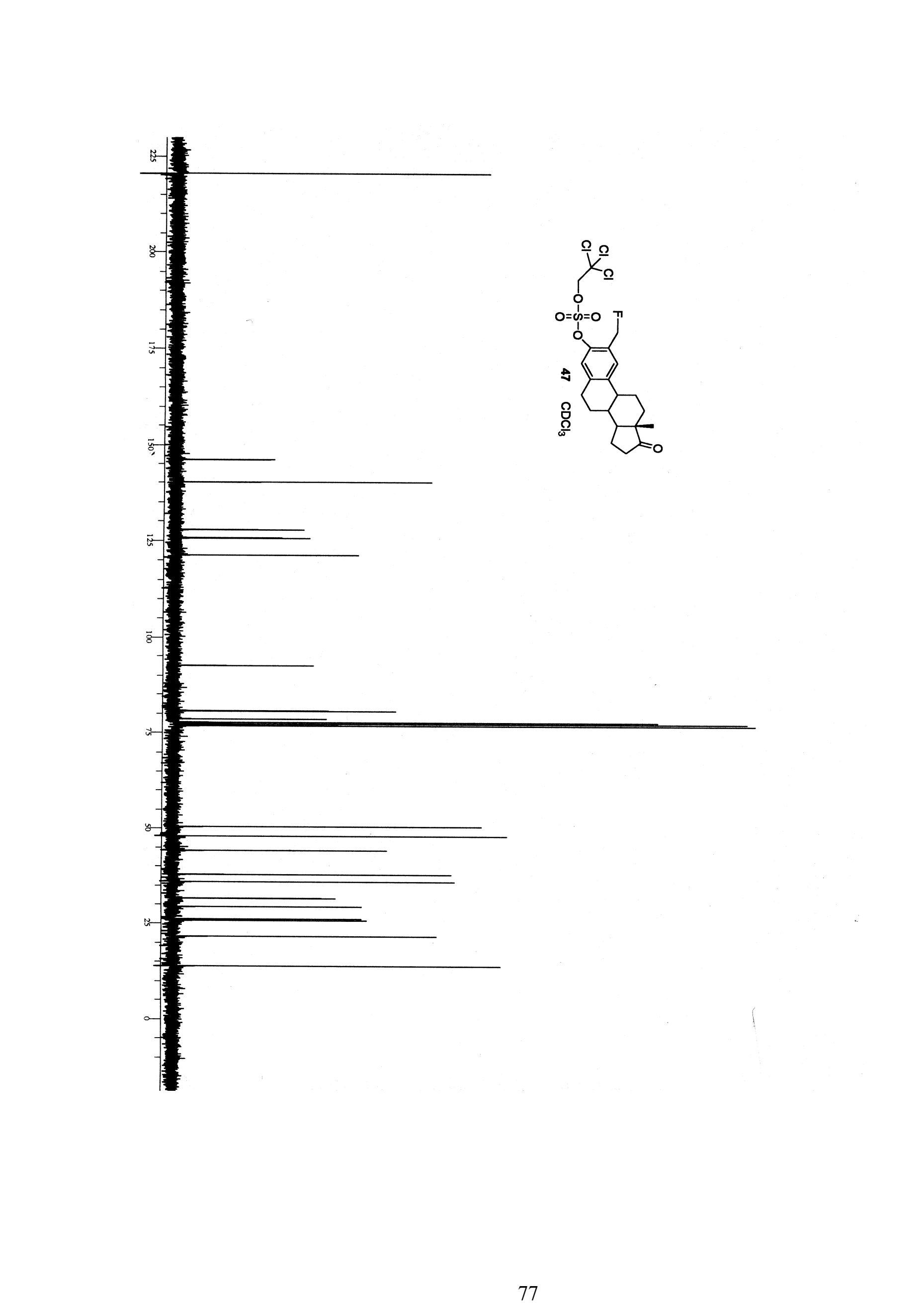




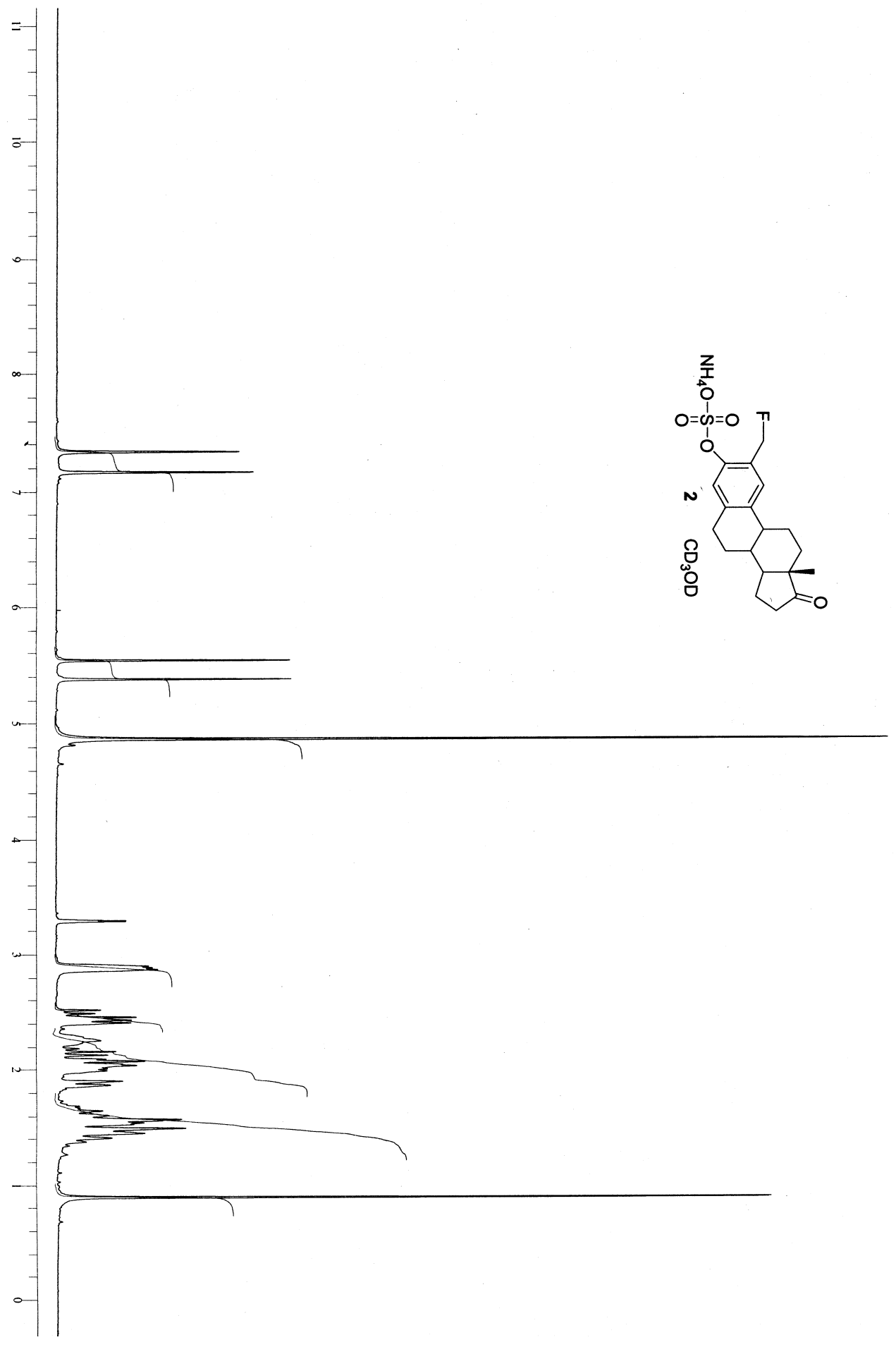




$$
F
$$




$$
\mid k
$$




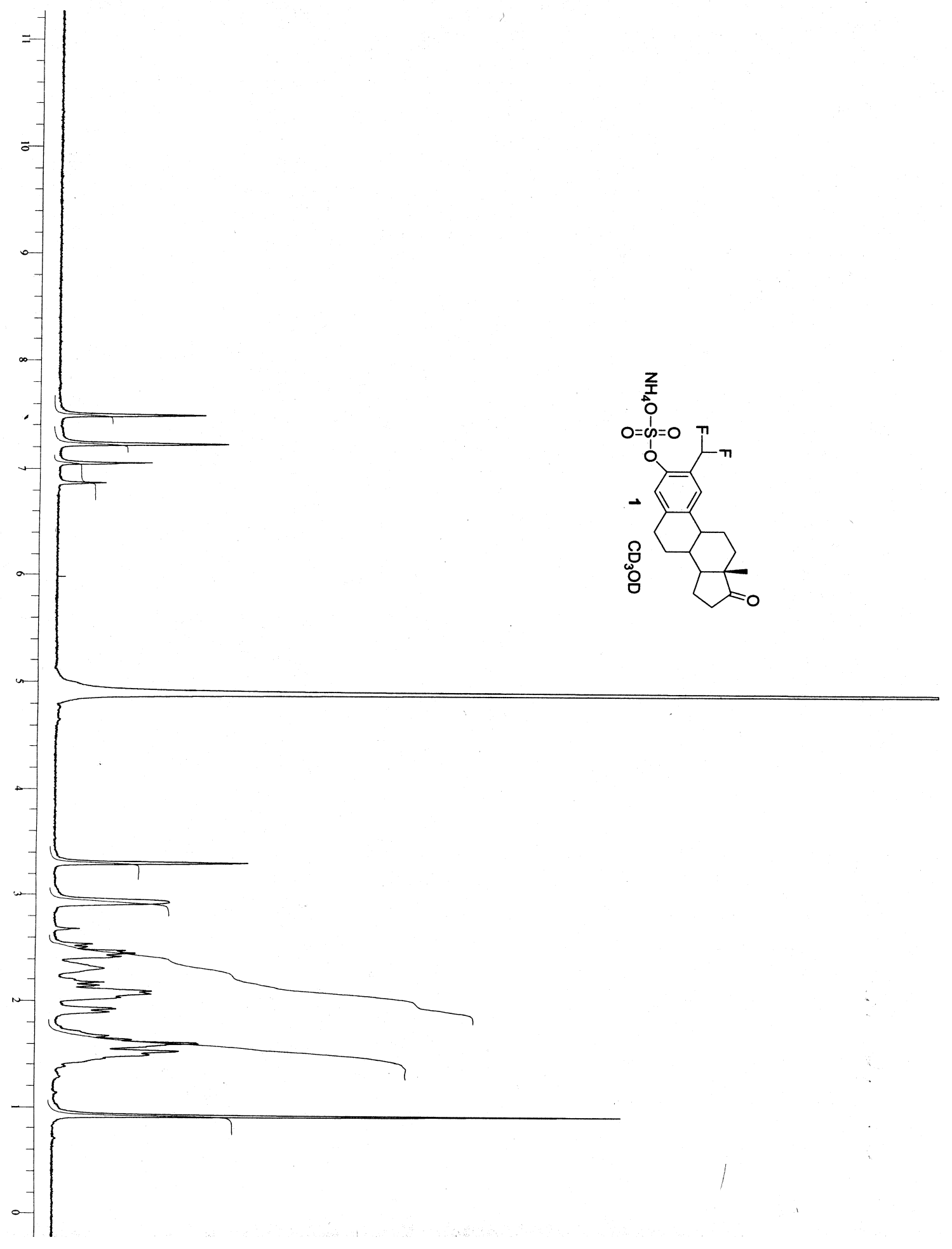




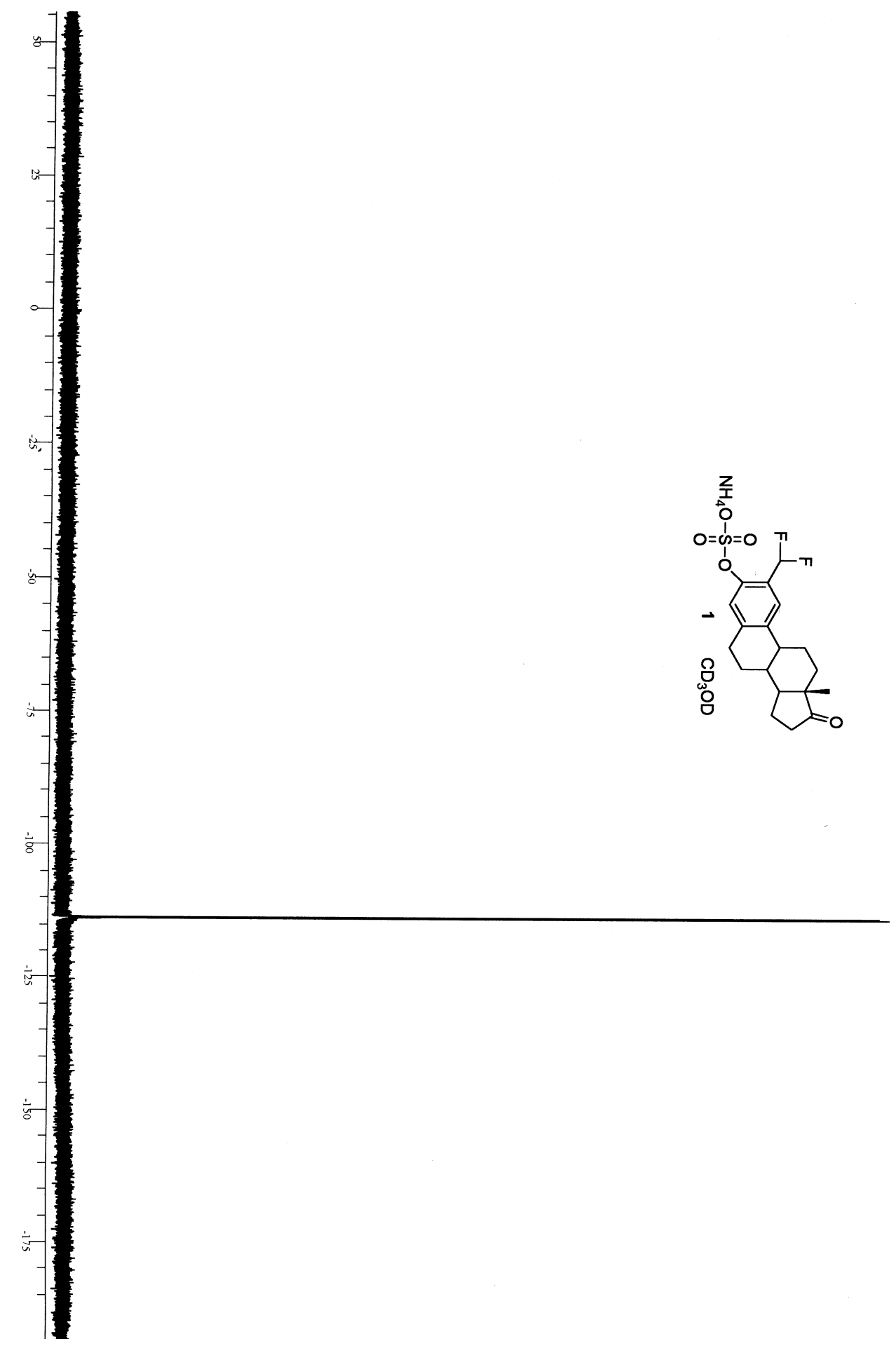

82 


$$
E=
$$




$$
E
$$

